\title{
THE FUNDAMENTAL SOLUTIONS OF THE HEAT EQUATIONS ON RIEMANNIAN SPACES WITH CONE-LIKE SINGULAR POINTS
}

\author{
By MASAYOSHI NAGASE
}

\section{$\S 0$. Introduction.}

The purpose of this paper is to derive some propertits of the fundamental solution of the initial-value problem

$$
\begin{aligned}
& \left(\frac{\partial}{\partial t}+\Delta\right) \theta(t, x)=0, \quad t>0 \\
& \lim _{t \downarrow 0} \theta(t, x)=\theta(x)
\end{aligned}
$$

for forms on a Riemannian space with cone-like singular points. Here the Laplacian $A$ is of Neumann or Dirichlet types, or, in certain cases, of unphysical types with ideal boundary conditions. We can show that the asymptotic expansion of the trace of the fundamental solution can have the $\log$ term and therefore the zeta function can have the simple pole at the origin; these new phenomena arising from the existence of the singular points will evoke much interest. In order to investigate them more closely, we will further study the same problem on the metric cone with the help of the Fourier integral operator theory.

The direction of this investigation has been first raised up by a short but pioneering paper due to J. Cheeger $([2])$. He attempted to extend the spectral geometric theory to the case where manifolds have singularities. The author's study substantially follows in his direction and should be started with carrying out the above basic research.

Notations and definitions: Before explaining the contents of this paper in detail, we will collect the general notations and definitions.

First, let $Y$ be a (perhaps, incomplete) oriented Riemannian manifold with $\widehat{\partial Y} \subset Y ; Y=$ Int $Y \cup \widehat{\partial Y}$ and, for the metric completion $\bar{Y}$,

$$
\hat{\partial Y}=\left\{y \in \bar{Y}-\operatorname{Int} Y: \begin{array}{l}
\bar{Y} \text { is a manifold with smooth boundary }\} \text { in some neighborhood of } y .
\end{array}\right.
$$

Let $A^{2}=\Lambda^{2}$ (Int $\left.Y\right)$ denote the space of smooth 2 -forms on Int $Y$ and let $\Lambda_{0}^{i}=\Lambda_{0}^{i}$ (Int $Y$ ) be the subspace of $\Lambda^{2}$ consisting of forms of compact support. Let $\Lambda^{2}(Y)$ be the subspace of $A^{2}$ consisting of forms which are smooth up to $\widehat{\partial Y}$. For

Recelved November 18, 1983 
l-forms $\theta_{1}, \theta_{2}$, let $\left\langle\theta_{1}, \theta_{2}\right\rangle_{y}$ be the pointwise inner product at $y \in Y$ defined by

$$
\theta_{1}(y) \wedge * \theta_{2}(y)=\left\langle\theta_{1}, \theta_{2}\right\rangle_{y} * 1_{y} .
$$

Here $*$ is the $*$-operator on $Y$. Moreover, by definining that the pointwise inner product of $i_{1}$ and $i_{2}$-forms $\theta_{1}$ and $\theta_{2}$ with $i_{1} \neq i_{2}$ is equal to 0 , the global inner product of forms $\theta_{1}$ and $\theta_{2}$ is defined by

$$
\left\langle\theta_{1}, \theta_{2}\right\rangle=\left\langle\theta_{1}, \theta_{2}\right\rangle_{Y}=\int_{Y} \theta_{1} \wedge * \theta_{z}=\int_{Y}\left\langle\theta_{1}, \theta_{2}\right\rangle_{y} * 1_{y} .
$$

Then, $L^{2} A^{2}=L^{2} A^{2}(Y)$ denotes the space of $i$-forms with finite norm:

$$
\|\theta\|=\|\theta\|_{Y}=\sqrt{ }\langle\theta, \theta\rangle,
$$

i. e. square integrable $i$-forms.

Let $d=d_{\imath}$ denote the exterior derivative on $\imath$-forms and $\delta=\delta_{i}$ be its formal adjoint; they should be regarded as unbounded linear operators on $L^{2} A^{*}$ by setting

$$
\begin{aligned}
& \text { dom } d_{i}=\left\{\theta \in A^{i} \cap L^{2} A^{\imath} \mid d \theta \in A^{2+1} \cap L^{2} A^{i+1}\right\}, \\
& \operatorname{dom} \delta_{i}=\left\{\theta \in A^{2+1} \cap L^{2} A^{2+1} \mid \delta \theta \in A^{2} \cap L^{2} \Lambda^{i}\right\}
\end{aligned}
$$

Let's set $d_{c, 2}=\left.d\right|_{A_{0}^{2}}$ and $\delta_{c, 2}=\left.\delta\right|_{A_{0}^{2+1}}$. Moreover the closures of $d_{2}, \delta_{i}, d_{c, l}$ and $\delta_{c, \imath}$ are denoted by $\bar{d}_{\imath}, \tilde{\delta}_{i}, \bar{d}_{c, \imath}$ and $\tilde{\delta}_{c, 2}$. Next, let $P=P(d, \delta)$ be a polynomial of $d, \delta$, and define

$$
\Lambda_{L}^{2}=\left\{\theta \in \Lambda^{2}(Y) \mid \text { For any } P, P \theta \in L^{2} \Lambda^{*} .\right\},
$$

$$
\begin{aligned}
& \Lambda_{a b s}^{2}=\left\{\theta \in \Lambda_{L}^{2} \mid \text { For any } P,\left.(* P \theta)\right|_{\hat{o} \hat{Y}}=0 .\right\}, \\
& \Lambda_{r e l}^{2}=\left\{\theta \in \Lambda_{L}^{2} \mid \text { For any } P,\left.(P \theta)\right|_{\hat{\partial} \hat{Y}}=0 .\right\},
\end{aligned}
$$

and set $d_{a b s}=d_{a b s, \imath}=\left.d\right|_{A_{a b s}^{2}}, d_{r e l}=d_{r e t, \imath}=\left.d\right|_{f_{r e t}^{2},}, \delta_{a b s}=\delta_{a b s, \iota}=\left.\delta\right|_{A_{a b s}^{2+1}}$ and $\delta_{r e l}=\delta_{r e l, 2}$ $=\left.\delta\right|_{A_{r e l}^{2+1}}$; their closures are denoted by $\bar{d}_{a b s}, \bar{d}_{r e l}, \bar{\delta}_{a b s}$ and $\tilde{\delta}_{r e l}$.

Moreover, several Laplacians are defined by

$$
\begin{aligned}
& \bar{J}_{N, \imath}=\bar{\delta}_{c, i} \bar{d}_{i}+\bar{d}_{l-1} \overline{\bar{o}}_{c, l-1}, \\
& \bar{J}_{D, \imath}=\tilde{\delta}_{i} \bar{d}_{c, i}+\bar{d}_{c, \imath-1} \bar{\delta}_{i-1} \text {, } \\
& \bar{J}_{a b s, \imath}=\bar{\delta}_{a b s, i} \bar{d}_{a b s, i}+\bar{d}_{a b s, \ell \cdots 1} \overline{\bar{\delta}}_{a b s, \imath-1} \text {, } \\
& \bar{J}_{r e l, \imath}=\overline{\tilde{o}}_{r e l, i} \bar{d}_{r e l, i}-\bar{d}_{r e l, 2-1} \overline{\bar{o}}_{r e l, l-1} \text {. }
\end{aligned}
$$

$J_{N}$ and $\bar{J}_{D}$ are called the Laplacians of Neumann and Dirichlet types, respectively; they are denoted by $A_{M}$ and $A_{L}$ in [7] and, from the facts

$$
\begin{aligned}
& \bar{d}_{c}^{*}=\overline{\hat{o}}, \\
& \bar{o}_{c}^{*}=\bar{d},
\end{aligned}
$$


they are self-adjoint operators. Here $\bar{d}_{c}^{*}$ and $\bar{\delta}_{c}^{*}$ are the Hilbert space adjoints of $\bar{d}_{c}$ and $\bar{\delta}_{c}$, respectively. By the way, we may remark that $\bar{J}_{a b s}$ and $\bar{J}_{r e l}$ are, in general, not self-adjoint; see Example 1.1. From now on, we set $\left(\Lambda_{b c}^{2}, \bar{d}_{b c, \imath}, \bar{\delta}_{b c, \imath}\right.$, $\left.\bar{J}_{b c, \imath}\right)=\left(\Lambda_{a b s}^{2}, \bar{d}_{a b s, \imath}, \bar{\delta}_{a b s, \imath}, \bar{J}_{a b s, \imath}\right)$ or $\left(\Lambda_{r e l}^{i}, \bar{d}_{r e l, \imath}, \bar{\delta}_{r e l, \imath}, \bar{\Delta}_{r e l, \imath}\right)$, provided that no confusions occur.

Second, we will define the metric cone. Let $N$ be a compact Riemannian manifold possibly with smooth boundary; the metric on $N$ is denoted by $\tilde{g}$. Set $\boldsymbol{R}^{+}=(0, \infty)$, then the space $\boldsymbol{R}^{+} \times N$ together with the Riemannian metric $d r \otimes d \boldsymbol{r}$ $+r^{2} \tilde{g}$ is called the metric cone over $N$, denoted by $C(N)$. Its metric completion is denoted by $C^{*}(N)=\{p\} \cup C(N)$ and the point $p$ is called the singular point of $C(N)$. Moreover the truncated cone $\left\{(r, \tilde{x}) \in C(N) \mid r_{1}<r<r_{2}\right\}$ is denoted by $C_{r_{1}, r_{2}}(N)$.

Third, among general $Y$ 's mentioned above, an $(m+1)$-dimensional Riemannian manifold $X$ which satisfies the following condition $(0.10)$ is called an $(m+1)$ dimensional Riemannian space with cone-like singular points:

(0.10) $\bar{X}$-Int $X$ equals to $\left\{p_{j}\right\}_{3}$, a set of finite points, called the singular pornts of $X$. Each point $p$, has a neighborhood $U$, in $\bar{X}$ which is isometric to a truncated cone $C_{0, u_{j}}^{*}\left(N_{j}^{m}\right)=\left\{p_{j}\right\} \cup C_{0, u_{j}}\left(N_{j}^{m}\right)$; the $U_{j}$ 's are disjoint from each other and the $N_{j}^{m}$ 's are $m$-dimensional compact Riemannian manifolds possibly with smooth boundary.

In the following, to simplify the notations, we set $N^{m}=\bigcup_{\jmath} N_{\jmath}^{m}$ and take $0<u \leqq$ $\min _{j} u_{j}$ and, without distinction, denote the subspace which is isometric to $C_{0, u}(N)$ through the above isometry, simply, by $C_{0, u}(N)$. For $0<\varepsilon \leqq u$, the complement of $C_{0, \varepsilon}(N)$ in $X$ is denoted by $X_{\varepsilon}$. Moreover, we write $X=C_{0, \varepsilon}(N) \cup X_{\varepsilon}$.

From now on, $X=C_{0, u}(N) \cup X_{u}$ always means an $(m+1)$-dimensional Riemannian space with cone-like singular points and $Y$ means a general Riemannian manifold mentioned above. In general, points of $X$ or $Y$ are denoted by $x, x_{1}$, $x_{2}$, etc. and, in particular, points of $C(N)=R^{+} \times N$ or $C_{0, u}(N)=(0, u) \times N$ are denoted by $x=(r, \tilde{x}), x_{1}=\left(r_{1}, \tilde{x}_{1}\right)$, etc. Moreover, we assume that, on $C_{0, u}(N)$, the orientation of $X$ is equal to the orientation which is defined by $d r \wedge \tilde{*} 1$, where $\tilde{*}$ is the $*$-operator on $N$.

Contents: We summarize the contents of the six sections making up this paper.

Section 1: We show that the Laplacian $\bar{A}_{b c, \imath}$ on $X$ is self-adjoint and $\bar{J}_{a b s, \imath}$ $=\bar{\Delta}_{N, \imath}, \bar{\Delta}_{r e l, \imath}=\bar{\Delta}_{D, \imath}$ except for the case where

(0.11) $\operatorname{dim} N=m=2 k$, the space of harmonic $k$-forms on $N$ satisfying the given boundary condition " $b c$ " is not equal to $\{0\}$, and $\imath=k$ or $k+1$.

In the excepted case, by introducing the new condition called "ideal boundary condition", we construct the self-adjoint Laplacians. Among them, the operators which equal to $\bar{A}_{N}$ or $\bar{A}_{D}$ are, of course, numbered. However, the other operators are also numbered among them; they are called unphysical Laplacians. 
Sections 2, 3: To make clear the fundamental solution of $(0.1)$, it is necessary to carry out the functional calculus on $C_{0, u}(N)$, therefore, on $C(N)$. In Section 2, we will calculate the formal eigenforms on $C(N)$. In Section 3 , on the basis of the results of Section 2, we will get the formal representation for the kernel of the function of our Laplacian on $C(N)$. We have generalized the representation [4.1] of [2] to the one for general forms.

Section 4: Let's assume that the metric completion $\bar{X}$ is compact. Then the Rellich-type theorem holds for $X$. Therefore, from the general theory of Fredholm, the Hodge decomposition exists and our Laplacians have the spectra consisting of eigenvalues of finite multiplicity. We will show, moreover, that the harmonic spaces are naturally identified with the de Rham-type cohomology groups attached to $X_{u}$. The results mentioned in this section have already been announced in [2] Theorems 2.1, 3.1.

Section 5: On the basis of the E.E. Levi's method, we will reconstruct the heat kernel on $X$ from the heat kernel $E_{C}$ on $C(N)$ and the heat kernel $E_{M}$ on a complement of some neighborhood of the singular point. $E_{C}$ and $E_{M}$ are accessible to us. Actually, $E_{M}$ is well known. Moreover, $E_{C}$ has the formal representation which can be written according to the result of Section 3, and, therefore, can be thoroughly investigated through the representation if we want to do so. Hence the reconstructed one is more accessible than the formal one. Once the reconstruction is accomplished, we can investigate the asymptotic expansion of the trace of the heat kernel. Moreover, as its immediate corollaries, we can derive the fundamental properties of the zeta function for $X$ and the asymptotic distribution of eigenvalues of our Laplacian.

By the way, the existence of the asymptotic expansion is also announced by J. Cheeger in [2] Theorem 5.1. He adopted the expression as follows: For $K>m+1$,

$$
\int_{X} \operatorname{tr} E_{\imath}(t) \sim \sum_{j=0}^{K} p \cdot f \cdot a_{j / 2} t^{-(m+1) / 2+j / 2}-\frac{1}{2}\left[\int_{N} a_{(m+1) / 2}(1, x)\right] \log t+\psi_{2}(N) .
$$

Here, $\operatorname{tr} E_{i}(t)$ is the pointwise trace of our heat kernel. Referring to [2] for the detailed explanations of notations, the constant term in the right hand side of (0.12) seems to have some difficulties to understand which occur because of the use of the index $K$. We have tried to express the asymptotic expansion more explicitly by making more clearly the term $\int_{N} \operatorname{tr} \varepsilon_{\imath}(1, x, 1, x, t)$ mentioned in [2]; it is denoted by $\operatorname{Tr}_{N} Z(t ; i)$ in this paper. Besides, in the last line but one in [2] $\$ 5$, it is asserted that the zeta function for $X$ can have double poles. However, according to our result, it has no double poles but can have a simple pole at the origin of $C$.

Section 6: As mentioned above, $E_{C}$ can be thoroughly investigated with the help of the formal representation. However, what we have really derived in Section 5 are only its fundamental properties, which are in fact good enough for the purpose of Section 5. By the way, for the purpose of making a further study of the trace of the heat kernel, that is, of comprehending the meanings of 
the coefficients of its asymptotic expansion, probably it will be useful to more closely investigate $E_{C}$ only with the help of the formal representation. Actually it will further offer new informations about the coefficients of the asymptotic expansion of the trace of $E_{M}$, too. In this section, we accordingly intend to study $E_{C}$ only with the help of the formal representation. Specifically, let $E_{C}\left(t, x_{1}, x_{2} ; i\right)$ denote the heat kernel $E_{C}$ for $i$-forms and let's define $Z\left(t, x_{1}, x_{2} ; i\right)$ by the formula

$$
E_{C}\left(t, x_{1}, x_{2} ; i\right)=\left(r_{1} r_{2}\right)^{(1+2(i-1)-m) / 2} e^{-\left(r_{1}-r_{2}\right)^{2} / 2} Z\left(t, x_{1}, x_{2} ; i\right),
$$

where $m=\operatorname{dim} \Lambda^{\prime}$ and $x_{j}=\left(r_{j}, \tilde{x}_{j}\right), y=1,2$. Then the pointwise traces have the following relations :

$$
\begin{aligned}
\operatorname{Tr} E(t,(r, \tilde{x}),(r, \tilde{x}) ; i) & =r^{2 t-(m+1)} \operatorname{Tr} Z(t,(r, \tilde{x}),(r, \tilde{x}) ; i) \\
& =r^{-1} \operatorname{Tr} Z\left(\frac{t}{r^{2}},(1, \tilde{x}),(1, \tilde{x}) ; i\right) .
\end{aligned}
$$

Therefore, for our purpose, it suffices to study $\operatorname{Tr} Z(t,(1, \tilde{x}),(1, \tilde{x}) ; i)$, that is, to characterize each coefficient of its asymptotic expansion by the invariants of a neighborhood of $\tilde{x}$ in $N$. Our aim is restricted to the case where $i=0$ and $\tilde{x} \in \operatorname{Int} N$. Accordingly, let's set

$$
\operatorname{tr} Z(t, \tilde{x})=\operatorname{Tr} Z(t,(1, \tilde{x}),(1, \tilde{x}) ; 0) .
$$

Then, observing further that $(0.15)$ is influenced mainly by the wave kernel for $N$, we can apply the Fourier integral operator theory to it. Reviewing that theory in Subsection 6.1, with the help of the key lemma mentioned in Subsection 6.2 , we will try to study $(0.15)$ in Subsection 6.3 .

The author is grateful to Professor D. Fujiwara and Professor K. Shiga for useful conversations during the preparations of this paper.

Finally, the author wants to emphasize once again that our methods depend heavily on J. Cheeger's works ([2], [3]).

\section{§1. Self-adjoint Laplacians; ideal boundary condition.}

Let $X=C_{0, u}(N) \cup X_{u}$ be an $(m+1)$-dimensional Riemannian space with conelike singular points. Then the Laplacian $\bar{J}_{b c, \imath}$ on $X$ is, in general, not selfadjoinnt. In this section, we will make a clear distinction between the two cases, i. e., the (first) case where it is self-adjoint and the (second) case where it is not self-adjoint. In the second case, we will produce the self-adjoint Laplacians by introducing the new conditions called the ideal boundary conditions.

Let us begin by adducing an example which shows that there exists the second case.

ExAmPle 1.1 (see Fig. 2.1). Assume that $X=C(N), \operatorname{dim} N=m=2 k$ and $\widetilde{\mathcal{F}}_{b c}^{k}(N)$ is not equal to $\{0\}$, where $\widetilde{\mathcal{F}}_{b c}^{b}(N)$ is the space consisting of harmonic 
$k$-forms on $N$ which satisfy the given boundary condition " $b c$ ". Given a form $\omega \in \widetilde{\mathscr{H}}_{b c}^{k}(N)$ with $\|\omega\|_{N}=1$, let's set $\Omega_{1}=\omega, \Omega_{2}=d r \wedge \omega$, which we regard as $k$ and $(k+1)$-forms on $C(N)$, respectively. Let $f_{1}$ and $f_{2}$ be smooth functions on $\boldsymbol{R}^{+}$ which satisfy $f_{1}(r)=r, f_{2}(r)=1$ for $r$ small, and $f_{1}(r)=f_{2}(r)=0$ for $r$ large. And we set

$$
\theta_{1,3}=\int_{i}(r) \Omega_{3},
$$

where $l=1,2, y=1,2$. Then $\theta_{l, 1}=\operatorname{dom} J_{b c, k}, \theta_{i, 2} \in \operatorname{dom} J_{b r k-1}$ and

$$
\begin{aligned}
& d \theta_{\imath, 1}=f_{i}^{\prime}(r) \Omega_{2}, \quad \partial \theta_{\ell, 1}=0, \quad J \theta_{2,1}=\cdots f_{l}^{\prime \prime}(r) \Omega_{1} .
\end{aligned}
$$

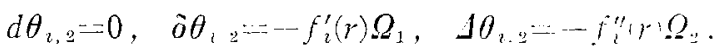

Therefore, we can easily show that

$$
\begin{aligned}
& \left\langle\Delta \theta_{1,1}, \theta_{2,1}\right\rangle \subset(x)-\left\langle\theta_{1,1}, \Delta \theta_{2,1}\right\rangle_{C(1)}=1 . \\
& \left\langle\Delta \theta_{1,2}, \theta_{2,2}\right\rangle_{C(x)}-\left\langle\theta_{1,2}, \Delta \theta_{2,2}\right\rangle_{C(x)}=1 .
\end{aligned}
$$

Thus $J_{b c, k}$ and $J_{b c, k+1}$ are not self-adjoint.

By changing this example slightly, we can easily understand that the Laplacian $J_{b c, 2}$ on $X$ is not self-adjoint in the case $(0.11)$ mentioned in $\$ 0$. To show that it is self-adjoint except for the case $(0.11)$, some preparations are needed.

For general $Y$, we have

LEMMA 1.2 ([6] Lemma 3.2).

$$
\bar{d}_{a b s}=\bar{d}, \quad \bar{\delta}_{r e l}=\bar{\delta} .
$$

Though it will be expected that $\bar{d}_{r e l}=\bar{d}_{c}$ and $\bar{\delta}_{a b s}=\bar{\delta}_{c}$, these are, in general, not valid. In fact we can offer an example which shows that $\bar{d}_{b c}^{*}+\bar{\delta}_{b c}$; as being easily understood from (0.9) and (1.3), $\bar{d}_{r e l}=\bar{d}_{c}$ and $\bar{\delta}_{a b s}=\bar{\delta}_{c}$ are respectively equivalent to $\bar{d}_{r e l}^{*}=\bar{\delta}_{r e l}$ and $\bar{d}_{a b s}^{*}=\bar{\delta}_{a b s}$. The example is as follows. In Example 1.1, we found $\Delta \theta_{i, 1}=\delta\left(f_{2}^{\prime} \Omega_{2}\right)$, thus we find $f_{2}^{\prime} \Omega_{2} \in$ dom $\bar{r}_{j_{k} k}$, but. from (1.2), we know $f_{2}^{\prime} \Omega_{2} \notin \operatorname{dom} \bar{d}_{b c, k}^{*}$.

We now search for the condition under which $\bar{d}_{b c}^{*}==\bar{n}_{i, c}$ is ralid. When

$$
\langle d \alpha, \beta\rangle_{Y}=\langle\alpha, \delta \beta\rangle_{Y}+\int_{\hat{\partial Y}} \alpha \wedge x_{i}
$$

holds for any $\alpha \in \mathcal{L}_{L}^{2}, \beta \subseteq \mathcal{A}_{L}^{L+1}$, we say that the $l$-L'-Stokes' thourem lor $Y$ holds.

LEMMA 1.3. If the t-L'-Stokes' theorem for $Y$ holds, then

$$
\bar{d}_{r e l, \imath}=\bar{d}_{c, \imath}, \quad \bar{\delta}_{a b s, i}=\overline{\bar{o}}_{c, \imath} \text {. }
$$

Proof. As above, it suffices to prove $\bar{d}_{b c, 2}^{*}=\bar{o}_{b c, 2} .(0.9)$ and 1.3$)$ imply that 
$\bar{d}_{b c, i}^{*} \subset \bar{\delta}_{b c, \imath}$ is always valid. Thus, it suffices to prove the converse implication. By a simple regularization argument, we have only to prove $d_{b c, \imath}^{*} \supset \delta_{b c, \imath}$. Given $\beta \in \operatorname{dom} \delta_{b c, \imath}$, it is trivial that $\left.(\alpha \wedge * \beta)\right|_{\hat{\partial Y}}=0$ for any $\alpha \in \operatorname{dom} d_{b c, \imath}$. Hence (1.4) implies $d_{b c, \imath}^{*} \supset \delta_{b c, 2}$.

Hence we get

COROLLARY 1.4. If the $(\imath-1), \imath$ - $L^{2}$-Stokes' theorems for $Y$ hold, then $\tilde{J}_{b c, 2}$ is self-adjoint, and, moreover, $\bar{J}_{a b s, \imath}=\bar{J}_{N, \imath}$ and $\bar{J}_{r e l, \imath}=\bar{J}_{D, \imath}$.

Here, let us return to our $X$. We will classify our study into three cases:

Case $A$ : the case except for Cases B, C,

Case $B: \operatorname{dim} N=2 k, \widetilde{\mathcal{H}}_{b c}^{k}(N) \neq\{0\}$ and $\imath=k$,

Case $C: \operatorname{dim} N=2 k, \widetilde{\mathcal{H}}_{b c}^{k}(N) \neq\{0\}$ and $i=k+1$.

In Cases $\mathrm{B}, \mathrm{C}$, we often specify a subspace $V$ of $\widetilde{\mathcal{H}}_{b c}^{k}(N)$; in the following, Cases $B(V), C(V)$ denote Cases $B, C$ with $V$ specified.

First we consider Case A. Theorem 2.2 of [4] extends immediately to our $N$ which may have a smooth boundary. Since $X_{u}$ is compact, this combined with [9] therefore yields that the $(i-1), i-L^{2}$-Stokes' theorems for $Y$ hold. Hence, from Corollary 1.4 , we have

Proposition 1.5. In Case $A, \bar{J}_{b c, \imath}$ is self-adjoint, and, moreover, $\bar{J}_{a b s, \imath}=\bar{J}_{\mathrm{N}, \imath}$ and $\bar{J}_{r e l, \imath}=\bar{J}_{D, 2}$.

Next, we consider Cases B, C ; in these cases, $\operatorname{dim} N=2 k$ and $\widetilde{\mathscr{T}}_{b c}^{k}(N) \neq\{0\}$. Then the $k-L^{2}$-Stokes' theorem does not hold; see the example following Lemma 1.2. Hence we need a certain device. According to [4], we will explain it.

Fix a subspace $V$ of $\widetilde{\mathscr{H}}_{b c}^{k}(N)$. Let $V^{\perp}$ denote the orthogonal complement of $V$ in $\widetilde{\mathscr{T}}_{b c}^{k}(\Lambda)$, and let's set $d r \wedge V=\{d r \wedge \omega \mid \omega \in V\}$ and $d r \wedge V^{\perp}=\left\{d r \wedge \omega \mid \omega \in V^{\perp}\right\}$. Let $\bar{V}, \bar{V} \perp, d r \wedge \bar{V}$ and $d r \wedge \bar{V}^{\perp}$ denote respectively the subspaces of $L^{2} A^{*}\left(C_{0, u}(N)\right)$ spanned by $V, V-d r \wedge V$ and $d r \wedge V^{\perp}$ with coefficients in $L^{2}((0, u))$. Moreover, for $\theta \in L^{2} \Lambda^{*}(X)$ let $\theta_{V}, \theta_{V^{\perp}}, \theta_{d r \wedge V}$ and $\theta_{d r \wedge V^{\perp}}$ denote the orthogonal projections of $\left.\theta\right|_{C_{0, u}(N)}$ to $\bar{V}, \bar{V} \perp, d r \wedge \bar{V}$ and $d r \wedge \bar{V}^{\perp}$, respectively. And we set

$$
\begin{aligned}
& A_{b c, l}^{k}(Y)=\left\{\theta \equiv A_{b c}^{b}(X) \mid \theta_{V \mid r=0}=(d \theta)_{d r_{i} \backslash V^{1} ; r=0}=(\delta d \theta)_{V \mid r=0}=\cdots=0\right\},
\end{aligned}
$$

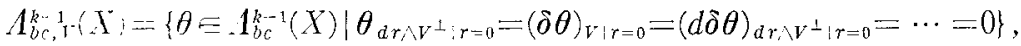

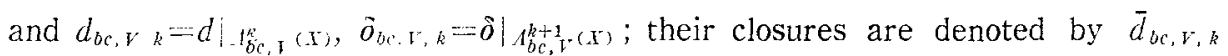
and $\bar{\delta}_{b c, V, k}$, respectively.

From the fact that

$$
(d \theta)_{d r, V^{\prime}}=d r \wedge \frac{\partial \theta_{V^{-}}}{\partial r}, \quad(\delta d \theta)_{V}=-\frac{\partial^{2} \theta_{V}}{\partial r^{2}},
$$

etc. in Case $B(T)$ which means Case $B$ with $V$ specified, the condition introduced newly in (1.6) is the one which is concerned only with $\theta_{V}+\theta_{V^{\perp}}$. Similarly, the 
one introduced in (1.7) is concerned only with $\theta_{a r \wedge V}+\theta_{d r \wedge V^{\perp}}$. Let's examine (1.6) closely. Let $\left\{\omega_{V, j}\right\}_{j}$ and $\left\{\omega_{V^{\perp}, j}\right\}_{\text {, }}$ be the orthonormal basis of $V$ and $V^{\perp}$, respectively. Then we can write

$$
\theta_{V}=\sum g_{V, j}(r) \omega_{V, j}, \quad \theta_{V^{\perp}}=\sum g_{V^{1}, j}(r) \omega_{V^{1}, j},
$$

and the new condition of (1.6) can be rewritten as follows:

$$
g_{V, 1}^{(2 n)}(0)=0, \quad g_{V^{\perp}, J}^{(2 n+1)}(0)=0, \quad n=0,1,2, \cdots .
$$

Now let's take $\theta \in \operatorname{dom} \bar{d}_{b c, k}$ and expand $\theta_{V}, \theta_{V^{\perp}}$ as (1.8). Then, $\theta \in \operatorname{dom} \bar{d}_{b c, V, k}$ means that each $g_{V, j}$ belongs to the Sobolov space $H_{0}^{1}\left(\boldsymbol{R}^{+}\right)$near $r=0$. Therefore, if we set

$$
\left\|\theta_{V}\right\|_{(r, r)}^{2}=\sum_{j}\left|g_{V, j}(r)\right|^{2}
$$

then

$$
\operatorname{dom} \bar{d}_{b c, Y, k}=\left\{\theta \in \operatorname{dom} \bar{d}_{b c, k} \mid\left\|\theta_{Y}\right\|_{(r, r)}=o(1), r \downarrow 0\right\} .
$$

Similarly, if we write, for $\theta \in \Lambda_{b c}^{k+1}(X)$,

$$
\theta_{d r \wedge V}=\sum f_{V, j}(r) d r \wedge \omega_{V, j}, \quad \theta_{d r \wedge V^{\perp}=\Sigma} \sum f_{V^{\perp}, j}(r) d r \wedge \omega_{V^{\perp}, j},
$$

then the new condition of (1.7) can be written as follows;

$$
f_{V, j}^{(2 n+1)}(0)=0, \quad f_{V^{\perp}, j}^{(2 n)}(0)=0, \quad n=0,1,2, \cdots .
$$

Thus, by using the norm defined by

$$
\left\|\theta_{d r \wedge V^{\perp}}\right\|_{(r, r)}^{2}=\sum_{j}\left|f_{V^{\perp}, j}(r)\right|^{2},
$$

we have

$$
\operatorname{dom} \bar{\delta}_{b c, \boldsymbol{V}, k}=\left\{\theta \in \operatorname{dom} \bar{\delta}_{b c, k} \mid\left\|\theta_{d r \wedge V^{\perp}}\right\|_{(r, r)}=0(1), r \downarrow 0\right\} .
$$

For the general definition and the elementary properties of the norm $\|\cdot\|_{(r, r)}$, refer to $(2.18)-(2.24)$.

The conditions introduced newly in (1.6), (1.7), (1.9), (1.11), (1.13) and (1.15) are generally called the ideal boundary conditions.

We have now

LEMMA 1.6. For any $\alpha \in \operatorname{dom} \bar{d}_{b c, V, k}$ and $\beta \in \operatorname{dom} \overline{\widetilde{o}}_{b c, V, k}$,

$$
\langle\bar{d} \alpha, \beta\rangle_{X}=\langle\alpha, \bar{\delta} \beta\rangle_{x} .
$$

Proof. Take $\alpha \in \operatorname{dom} d_{b c, V, k}$ and $\beta \in \operatorname{dom} \delta_{b c, V, k}$. Then it suffices to prove (1.16) for these $\alpha, \beta$. Since

$$
\int_{X_{\varepsilon}} d \alpha \wedge * \beta=\int_{X_{\varepsilon}} \alpha \wedge * \delta \beta-\int_{(\varepsilon, N)} \alpha \wedge * \beta,
$$


it suffices to search for a sequence $\left\{\varepsilon_{s}\right\}, \varepsilon_{s} \downarrow 0$ such that

$$
\int_{\left(s_{s}, v\right)} \alpha \wedge * \beta \rightarrow 0
$$

The way of proof is similar to that of [4] Theorem 2.2. Set

$$
\alpha=\phi_{1}+d r \wedge \omega_{1}, \quad * \beta=\phi_{2}+d r \wedge \omega_{2},
$$

where $\phi_{n}, \omega_{2}$ do not involve $d r$. Then the ideal boundary conditions yield

$$
\phi_{1, V i r=0}=\phi_{2, \tilde{*}^{\perp}{ }_{i r=0}}=0 \text {. }
$$

According to the Hodge decomposition for $N$

$$
L^{2} \Lambda^{k}(N)=\overline{d \Lambda_{b c}^{k-1}(N)} \oplus \widetilde{\mathcal{H}}_{b c}^{b}(N) \oplus \overline{\delta \Lambda_{b c}^{k+1}(N)},
$$

we decompose $\phi$, as follows; for $r$ fixed,

$$
\dot{\phi}_{J}=\phi_{3, \tilde{\varepsilon}}+\phi_{3, \tilde{x}}+\phi_{j, \tilde{c}} \text {. }
$$

Then we have

$$
\begin{aligned}
& \int_{(\varepsilon, y)} \alpha \wedge * \beta=\int_{(\varepsilon, x)} \phi_{1} \wedge \phi_{2} \\
& =\int_{(\varepsilon, N)} \phi_{1, Y} \wedge \phi_{2, \tilde{*} V}+\int_{(\varepsilon, N)} \phi_{1, V^{\perp}} \wedge \phi_{2, \tilde{*} V^{\perp}} \\
& \quad+\int_{(\varepsilon, N)} \phi_{1, \tilde{e} \wedge} \wedge \phi_{2, \tilde{e}}+\int_{(\varepsilon, N)} \phi_{1, \tilde{e} \wedge \phi_{2, \tilde{e}}}
\end{aligned}
$$

Hence (1.20) and the estimations (2.63) and (2.64) of [4] yield the existence of the desired sequence $\left\{\varepsilon_{s}\right\}$.

LEMMA 1.7. $\quad \bar{d}_{b c, V, k}^{*}=\overline{\bar{o}}_{b c, V, k}$.

Proof. Consider first the case " $b c "=" a b s "$. We will prove $\vec{d}_{a b s, \boldsymbol{V}, k}=\bar{\delta}_{a b s, V, k}^{*}$. Since Lemma 1.6 implies $\bar{d}_{a b s, V, k} \subset \bar{\delta}_{a b s, V, k}^{*}$, it suffices to prove the converse implication. Obviously

$$
\bar{\delta}_{a b s, \nu, k}^{*} \subset \bar{\delta}_{c, k}^{*}=\bar{d}_{k}=\bar{d}_{a b s, k}
$$

follows from (0.9) and (1.3). Hence, if $V=\{0\}$, then the proof is complete. In the following, we assume that $V \neq\{0\}$. Take $\alpha \in \operatorname{dom} \bar{\delta}_{a b s, V, k}^{*}$. Moreover fix $\beta \in \operatorname{dom} \bar{\delta}_{\alpha b s, k, k}$ arbitrarily. Then (1.24) implies that $\bar{\delta}^{*} \alpha=\bar{d} \alpha$ and $\langle\alpha, \bar{\delta} \beta\rangle_{X}=$ $\langle\bar{d} \alpha, \beta\rangle_{X}$. Hence, observing (1.17), we have, for almost all $\{\varepsilon\}$ with $\varepsilon \downarrow 0$,

$$
\int_{(\varepsilon, N)} \alpha \wedge * \beta \rightarrow 0
$$

when $\varepsilon ! 0$. Now expand $\alpha, * \beta$ into the sums (1.19) and, moreover, expand $\phi_{j}$ 
into the sum (1.22). Hence (1.25) equals to (1.23). Moreover, the ideal boundary condition and the estimations (2.63) and (2.64) of [4] imply that the second, third and fourth terms of (1.23) tend to 0 when $\varepsilon ! 0$ for almost all $\{\varepsilon\}$ with $\varepsilon \downarrow 0$. This combined with (1.25) implies that, for almost all $\{\varepsilon\}$ with $\varepsilon \downarrow 0$, we have

$$
\lim _{\Sigma \downarrow 0} \int_{(s, Y)} \phi_{1, V} \wedge \phi_{2, \tilde{*} V}=0
$$

By the way, if $\omega$ is an element of $V$ with $\|\omega\|_{N}=1$ and $f$ is a smooth function whose restrictions to $X_{u}$ and some neighborhood of the singular point are respectively equal to 0 and 1 , then $\beta=f d r \wedge \omega$ belongs to $\operatorname{dom} \bar{\delta}_{a b s, v, k}$. For such $\beta$, (1.26) can be rewritten as follows.

$$
\left\langle\dot{\phi}_{1, V}(\varepsilon, \tilde{x}), \omega(\tilde{x})\right\rangle_{N \rightarrow 0}
$$

Hence, by expanding $\phi_{1, r}$ in terms of an orthonormal basis of $V$, we can easily show that $\left\|\alpha_{V}\right\|_{(r, r)}=\left\|\phi_{1, v}\right\|_{(r, r)}=o(1)$. That is, $\alpha \in \operatorname{dom} \bar{d}_{a b s, V, k}$.

In the case where " $b c "=$ " $a b s "$, the lemma is thus proved. For the lemma in the case where " $b c "=$ "rel", it suffices to prove $\vec{d}_{r e l, V, k}^{*} \subset \bar{\delta}_{r e l, V, k}$. This is similarly proved.

Now, we define the new Laplacians as follows;

$$
\begin{aligned}
& J_{b c, V, k}=\bar{\delta}_{b c, V, k} \bar{d}_{b c, V, k}+\bar{d}_{b c, k-1} \bar{\delta}_{b c, k-1}, \\
& J_{b c, V, k+1}=\bar{\delta}_{b c, k+1} \bar{d}_{b c, k+1}+\bar{d}_{b c, V, k} \bar{\delta}_{b c, V, k} .
\end{aligned}
$$

Then [7] Theorem 1.1, (0.9), (1.3), Lemma 1.7, and the facts that $\bar{d}_{b c,\{0\}, k}=\bar{d}_{b c, k}$ and $\tilde{\delta}_{b c, \ddot{H}_{b c}^{k}(N), k}=\tilde{\delta}_{b c, k}$ yield

Proposition 1.8. In Cases $B, C, J_{b c V, \imath}$ is self-adjoint, and, moreover, $\bar{J}_{a b s,(0), i}=\bar{J}_{N, \imath}$ and $\bar{J}_{r e l, \tilde{H}_{b c}^{k}(N), \iota}=\bar{J}_{D, \imath}$.

The new Laplacians which are identified with neither $\bar{J}_{N, \imath}$ nor $\bar{J}_{D, \imath}$ will be called the unphysical Laplacians.

From now on, to simplify the notations, we set

$$
J_{2}= \begin{cases}J_{b c, r} & \text { in Case } A, \\ J_{b c, V,} & \text { in Cases } \mathrm{B}(V) \text { or } \mathrm{C}(V)\end{cases}
$$

\section{§. Formal eigenforms on the metric cone.}

Let $N$ be an $m$-dimensional compact Riemannian manifold possibly with smooth boundary.

The eigenforms of the Laplacian on $C(N)$, namely, the metric cone over $N$, are calculated in [2]. However, the results mentioned in [2] are rather hard to read and understand and have some oversights; for example, [3.9] of [2]. (This oversight has already been corrected in the revised version of [3] (1980).) Since 
the functional calculus on our $X$ is based on these results, it will be better to rearrange them once more. The proofs are perfectly based on the elementary calculations. Hence we will omit them; refer to [15] Appendix.

Let $\tilde{*} \tilde{d}, \tilde{\delta}, \tilde{d}$, be the intrinsic operators on $N$ and $*, d, \delta, \Delta$ be those on $C(N)$.

Let $\theta(r, \tilde{x})=g(r) \phi(\tilde{x})+f(r) d r \wedge \omega(\tilde{x})$ be an $i$-form on $C(N)$, where $\phi$ and $\omega$ are $\imath$ and $(i-1)$-forms which do not involve $d r$. Then we have

$$
\begin{gathered}
* \theta=(-1)^{2} r^{m-22} g d r \wedge \tilde{*} \phi+r^{m-2(i-1)} f \tilde{*} \omega, \\
d \theta=g d \phi+d r \wedge\left(g^{\prime} \phi-f \tilde{d} \omega\right), \\
\delta \theta=r^{-2} g \tilde{\delta} \phi+\left\{-f^{\prime}-(m-2(i-1)) r^{-1} f\right\} \omega-r^{-2} f d r \wedge \tilde{\delta} \omega, \\
\Delta \theta=\left\{-g^{\prime \prime}-(m-2 i) r^{-1} g^{\prime}\right\} \phi+r^{-2} g \tilde{\Delta} \phi-2 r^{-3} g d r \wedge \tilde{\delta} \phi \\
+\left\{-f^{\prime \prime}-(m-2(i-1)) r^{-1} f^{\prime}+(m-2(i-1)) r^{-2} f\right\} d r \wedge \omega \\
+r^{-2} f d r \wedge \tilde{\Delta} \omega-2 r^{-1} f \tilde{d} \omega .
\end{gathered}
$$

We have the Hodge decomposition

$$
A_{b c}^{2}(N)=d \Lambda_{b c}^{2-1}(N) \oplus \tilde{\mathscr{H}}_{b c}^{2}(N) \oplus \tilde{\tilde{o}} \Lambda_{b c}^{2+1}(N),
$$

where $\widetilde{\mathscr{T}}_{b c}^{a}(N)$ is the space consisting of harmonic $i$-forms on $N$ which satisfy the given boundary condition "bc"= "abs" or "rel". Let $\left\{\omega_{b c, \tilde{e}(i), j}\right\}_{\nu},\left\{\omega_{b c, \tilde{\mathscr{H}}(i), j}\right\}_{,}$, $\left\{\omega_{b c, \tilde{e}(i), j}\right\}$, be those orthonormal basis of $\overline{\bar{d} \Lambda_{b c}^{2-1}(N)}, \widetilde{\mathscr{H}}_{b c}^{z}(N), \overline{\tilde{\delta} \Lambda_{b c}^{2+1}(N)}$ which are consisting of the eigenforms of $\tilde{\Delta}_{b c}$, namely, the Laplacian together with the given boundary condition " $b c$ ". Let $\left\{\mu_{b c, \tilde{e}(i), j}\right\}_{\jmath},\left\{\mu_{b c, \tilde{\mathscr{H}}(i), j}(=0)\right\}_{\jmath},\left\{\mu_{b c, \tilde{c}(i), j}\right\}_{j}$ be the sets of corresponding eigenvalues, respectively. Here each set has the order relation: $\mu_{b c, \tilde{e}(i), \jmath} \leqq \mu_{b c, \tilde{e}(i), j+1}$ for any $\jmath$, etc. Besides, according to circumstances, the objects of " $\tilde{\ell}(i)$ " and " $\widetilde{\mathscr{H}}(i)$ " types are gathered and rearranged as follows; $\left\{\left(\omega_{b c, \tilde{c}(i), \jmath}, \mu_{b c, \tilde{c}(i), j}\right)\right\}$, with the order relation where $\mu_{b c, \tilde{c}(i), j} \leqq \mu_{b c, \tilde{c}(i), j+1}$ for any $j$. Similarly the objects of " $\widetilde{\mathcal{H}}(i)$ " and " $\tilde{c}(i)$ " types are gathered and rearranged as follows ; $\left\{\left(\omega_{b c, \tilde{c c}(i), \jmath}, \mu_{b c, \tilde{c}(i), j}\right)\right\}$, with the order relation where $\mu_{b c, \tilde{c c}(i), j} \leqq \mu_{b c, \tilde{c c}(i), j+1}$ for any $\jmath$.

From now on, unless confusions occur, we will sometimes omit the subscripts $b c, \tilde{e}(i)$, etc.

We can now expand an $\imath$-form $\theta(r, \tilde{x})$ as follows :

$$
\begin{aligned}
\theta= & \sum_{j} g_{\tilde{c}(i), j}(r) \omega_{\tilde{c} \tilde{c}(l), j}(\tilde{x}) \\
& +\sum_{j}\left\{g_{\tilde{c \tilde{e}(i-1), j}, j}(r) d \omega_{\tilde{c}(i-1), j}(\tilde{x})+f_{\tilde{c}(i-1), j}(r) d r \wedge \omega_{\tilde{c} \tilde{e}(i-1), j}(\tilde{x})\right\} \\
& +\sum_{j} f_{\tilde{c}(i-1), j}(r) d r \wedge \omega_{\tilde{c}(i-1), j}(\tilde{x}) .
\end{aligned}
$$

Here we have omitted the subscript " $b c$ ". Then, from (2.4), $\Delta \theta$ can be expanded as follows: 


$$
\begin{aligned}
& J \theta=\sum_{j}\left\{-g_{\tilde{c}(i), J}^{\prime \prime}-(m-2 i) r^{-1} g_{\tilde{c}(i), J}^{\prime}+\mu_{\tilde{c} \tilde{c}(i),} \gamma^{-2} g_{\tilde{c} \tilde{c}(i), j}\right\} \omega_{\tilde{c} \tilde{c}(i), J} \\
& +\sum_{j}\left[\left\{-g_{\tilde{c}(i-1), j}^{\prime \prime}-(m-2 i) r^{-1} g_{\tilde{c}(i-1), j}^{\prime}+\mu_{c e}(i-1), j r^{-2} g_{\tilde{c}(i-1), j}\right.\right. \\
& \left.-2 r^{-1} f_{\tilde{c} \tilde{e}(i-1), j}\right\} d \omega_{\tilde{c} \tilde{e}(i-1), j}+\left\{-f_{\tilde{c} \tilde{e}(i-1), j}^{\prime \prime}-(m-2(i-1)) r^{-1} f_{\tilde{c} \tilde{e}(i-1),}^{\prime}\right. \\
& +(m-2(i-1)) r^{-2} f_{\tilde{c} \tilde{e}(i-1), j}+\mu_{\tilde{c} \tilde{e}(i-1), j} r^{-2} f_{\tilde{c} \tilde{e}(i-1), j} \\
& \left.-2 \mu_{\tilde{\tilde{e}(i \sim 1), \jmath}} r^{-3} g_{\tilde{c \tilde{e}(2-1)}, j}\right\} d r \wedge \omega_{\tilde{c} \tilde{e}(i-1), J]} \\
& +\sum_{j}\left\{-f_{\tilde{c}(i-1), j}^{\prime \prime}-(m-2(i-1)) r^{-1} f_{\tilde{c}(i-1), j}^{\prime}+(m-2(i-1)) r^{-2} f_{\tilde{c}(i-1), \jmath}\right. \\
& \left.+\mu_{\tilde{\varepsilon}(i-1), j} r^{-2} f_{\tilde{c}(\imath-1), j}\right\} d r \wedge \omega_{\tilde{c}(i-1), \jmath} .
\end{aligned}
$$

Therefore the differential equation $\Delta \theta=\lambda^{2} \theta$ can be reduced to the simultaneous differential equations of Euler-types with respect to the coefficients $g_{\tilde{c} \tilde{c}(i), j}(r)$, $g_{c \tilde{e}(i-1), j}(r)$, etc. appeared in (2.6). By solving these differential equations, we get the following two lemmas.

Set

$$
\begin{aligned}
& \alpha(i)=(1+2 l-m) / 2, \\
& \nu(\tilde{c c}(i), k)_{\jmath}=\sqrt{\mu_{c \tilde{c}(i), j}+\alpha(k)^{2}}, \\
& \nu(\tilde{c c}(i))_{j}=\nu(\tilde{c c}(i), i)_{j}, \\
& a^{ \pm}(\tilde{c c}(i), k)_{\jmath}=\alpha(k) \pm \nu(\tilde{c c}(i), k)_{\jmath}, \\
& a^{ \pm}(\tilde{c c}(i))_{3}=a^{ \pm}(\tilde{c c}(i), i)_{2},
\end{aligned}
$$

etc. If necessary to indicate the given boundary condition " $b c$ ", we will use such notations as $\nu(b c, \tilde{c c}(i), k)_{\jmath}, a^{ \pm}(b c, \tilde{c c}(i), k)_{\jmath}$, etc.

LEMMA 2.1. An i-form $\theta$ which satisfies $\Delta \theta=0$ can be uritten as a (convergent) sum of forms of the following four types:

$\left.(2.9)_{\tilde{c} \tilde{c}(i), \jmath}^{\stackrel{ \pm}{\leftrightarrows}} \quad r^{a \neq(\tilde{c}(i))}\right) \omega_{c \tilde{c}(\imath), j}(\tilde{x})$,

$\left.(2.10)_{\tilde{c e}(i-1), \jmath}^{ \pm} \quad d\left(r^{a \doteq(\tilde{e}(i-1))}\right) \omega_{\tilde{e}(i-1), j}(\tilde{x})\right)$,

$(2.11)_{\tilde{c} \tilde{e}(i-1), \jmath}^{ \pm} \quad r^{a^{ \pm}(\tilde{c}(i-1)) j^{+2}} \tilde{d} \omega_{\tilde{c} \tilde{e}(i-1), j}(\tilde{x})+a^{\tilde{*}}(\tilde{c e}(i-1)), r^{a^{ \pm}(\tilde{c}(i-1))_{j+1}} d r \wedge \omega_{\tilde{c e}(i-1), j}(\tilde{x})$,

$(2.12)_{\tilde{c}(\imath-1), \jmath}^{ \pm} \quad r^{a^{ \pm}(\tilde{c}(i-1), \imath-2)_{j}+1} d r \wedge \omega_{\tilde{c}(i-1), j}(\tilde{x})$,

where $(2.9)^{-},(2.11)^{-}$and $(2.12)^{-}$should be multiplied by $\log r$ if $\nu(\widetilde{c c}(i))_{3}=0$, $\nu(\tilde{c} \tilde{e}(i-1))_{j}=1$ and $\nu(\widetilde{c}(i-1), i-2)_{j}=0$, respectively.

LEMMA 2.2. Set $\lambda>0$. An 2 -form $\theta$ whach satisfies $\Delta \theta=\lambda^{2} \theta$ can be written as a (convergent) sum of forms of the following four types: 
$(2.13) \tilde{\tilde{c}(1), j} \quad r^{n(i)} J_{ \pm v(\tilde{c}(i))}(\lambda r) \omega_{\tilde{c}(i), \jmath}$,

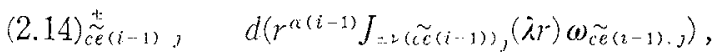

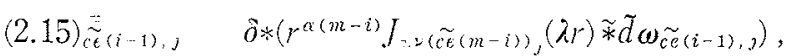

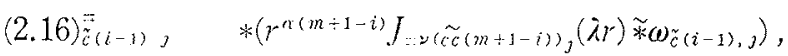

where the Bessel function $J_{-, j}(\lambda r)$ should be replaced by the Veumann function $V_{\nu j}(\lambda r)$ if $\nu_{j}$ is a non-negative integer.

In $(2.15)$ and $(2.16)=$, the eigenvalues used for defining $\nu(\tilde{c}(m-i))$, and $\nu(\tilde{c c}(m+1-i))$, are respectively those of $\widetilde{*} \tilde{d} \omega_{\tilde{c} \tilde{e}(i-1),}$, and $\widetilde{*} \omega_{\tilde{c}(i-1),}$, which satisfy the boundary condition adjoint to ours " $b c$ ". Hence, if we promise to interpose " $a d b c$ " in order to specify that the boundary condition under consideration is adjoint to the one " $b c$ ", we should replace $\nu(\tilde{c e}(m-i))_{3}$ and $\nu(\tilde{c c}(m+1-i))_{3}$ by $\vee(a d b c, \tilde{c}(m-i))_{3}$ and $2(a d b c, \tilde{c} c(m-1-i))_{3}$, respectively. Remark that

$$
\begin{aligned}
& \nu(a d b c, \widetilde{c e}(m-i))_{3}=\nu(b c, \widetilde{c e}(i-1))_{3}, \\
& \nu(a d b c, \widetilde{c}(m-1-i))_{3}=\nu(b c, \tilde{c}(i-1), l-2)_{2} .
\end{aligned}
$$

From (2.1) $-(2.3)$ and this fact (2.17), we can rewrite $(2.14)-(2.16)$ as follows.

$$
\begin{aligned}
& r^{a(i-1)} J_{=2(\tilde{e} \tilde{e}(2-1))}(\lambda r) d \omega_{(\tilde{e}(i-1), 3} \\
& +\left\{\alpha(i-1) r^{a(i-1)-1} J_{: i L(\widetilde{e}(i-1)){ }_{j}}(\lambda r)\right.
\end{aligned}
$$

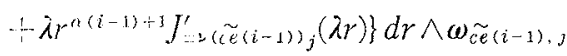$$
(-1)^{m(i-1)+1}\left[\left\{-\alpha(i-1) r^{(r(2-1)} J_{ \pm \nu(\tilde{c}(i-1))_{j}}(\lambda r)\right.\right.
$$$$
\left.\therefore \lambda r^{n(i-1)+1} J_{=\nu(\tilde{e}(i-1))_{j}}^{\prime}(\lambda r)\right\} \tilde{d} \omega_{c \tilde{e}(i-1),}
$$$$
-\mu_{c \tilde{\varepsilon}(i-1), j} r^{(r(2-1)-1} J_{ \pm \nu(\tilde{\varepsilon} \tilde{e}(i-1))}(\dot{\lambda} r) d r \wedge \omega_{c \tilde{\varepsilon}(i-1), j]}
$$

$$
(-1)^{m} \gamma^{(i(2-1)} J_{:=\nu(\tilde{c}(i-1), 2-2),}(\lambda r) d r \wedge \omega_{\tilde{c}(i-1), 3} .
$$

These formulas are also useful for various estimations.

Next, we will investigate the square-integrabilities of $(2.9)-(2.16)$ near the singular point. The results are collected into Figures 2.1, 2.2. We will confine ourselves to the explanation of the way from which we can know whether they are square-integrable or not.

Let $\theta(r, \tilde{x})=\theta_{1}(r, \tilde{x})+d r \wedge \theta_{2}(r, \tilde{x})$ and $\eta(r, \tilde{x})=\eta_{1}(r, \tilde{x})-d r \wedge \eta_{2}(r, \tilde{x})$ be $i-$ forms, where $\theta$, and $\eta_{0}$ do not involve $d r$. Set

$$
\begin{gathered}
\left\langle\theta, \gamma_{i}\right\rangle\left\langle(s, r)=\int_{(s, y)}\left\{\theta_{1}(r, \tilde{x}) \wedge \widetilde{*}_{s} \eta_{1}(r, \tilde{x})+\theta_{2}(r, \tilde{x}) \wedge \widetilde{*}_{s} \gamma_{2}(r, \tilde{x})\right\},\right. \\
\|\theta\|_{(s, r)}=\sqrt{\langle}\langle\theta, \theta\rangle \overline{(s, r)} .
\end{gathered}
$$


Here $\widetilde{*}_{s}$ is the $*$-operator on $(s, N)$ with metric $s^{\prime \prime} \tilde{g}$. Then we have the following formulas:

$$
\begin{aligned}
& \left\|\theta_{1}\right\|_{(r, r)}=r^{m n-2}\left\|\theta_{1}\right\|(1, r), \\
& \left\|d r \wedge \theta_{2}\right\|_{(r, r)}=\left\|\theta_{2}\right\|_{(r, r)}=r^{m-2}-(2-1)\left\|\theta_{2}\right\|(1, r) \text {, } \\
& \|\theta\|_{C_{0,8}(N)}^{2}=\int_{0}^{*}\left\{\left\|\theta_{1}\right\|_{(r, r)}^{2},-\left\{\theta_{,} \|_{(r,}^{2}\right\} d r .\right.
\end{aligned}
$$

Therefore, if $\theta(r, \tilde{x})=g(r) \phi(\tilde{x})+f(r) d r \wedge \omega(\tilde{x})$, then

$$
\|\theta\|_{c_{0, z}}^{2}(v)=\|\phi\|_{N}^{2} \int_{0}^{\varepsilon} r^{m-2 l} g(r)^{2} d r+\|\omega\|_{N}^{2} \int_{11}^{\varepsilon} r^{m-2(l-1)} f(r)^{2} d r .
$$

\begin{tabular}{|c|c|c|c|c|}
\hline & $\begin{array}{c}L^{2} L^{2}\left(C_{0, z}\right) \\
\cdot \\
\cdot\end{array}$ & $\begin{array}{c}L^{2} A^{2+1}\left(C_{0, z}\right) \\
\Psi^{u} \\
d\end{array}$ & $\begin{array}{c}L^{2} \lambda^{(3)}\left(C_{0.3}\right) \\
\delta\end{array}$ & $\begin{array}{c}(4) \\
\text { For any } \\
P=P(d, \delta), \\
P \cdot \in L^{2} \Lambda^{*}\left(C_{0 . s}\right)\end{array}$ \\
\hline$(2.9)^{+}$ & 0 & $0^{(\dagger)_{1}}$ & (C) & 0 \\
\hline$(2.9)^{-}$ & iff ${ }_{y_{j}<1}^{\bigcirc}$ & iff $2 i \geqq m \& \mu_{\mu_{j}=0}^{(\dagger)_{2}}$ & (C) & iff $2 l=m \& \mu_{\jmath}=0$ \\
\hline$(2.10)^{\perp}$ & 0 & ; & (C) & 0 \\
\hline & & i & & \\
\hline$(2.10)^{-}$ & x & (C) & (C) & X \\
\hline$(2.11)^{+}$ & 0 & 0 & 0 & 0 \\
\hline$(2.11)^{-}$ & iff ${ }_{\nu_{j}<2}<2$ & iff $\stackrel{\bigcirc}{\nu_{j}<1}$ & iff $\underset{\nu_{j}<1}{\bigcirc}$ & x \\
\hline$(2.12)^{+}$ & 0 & (c) & $0^{(\dagger)_{1}}$ & 0 \\
\hline .12$)$ & iff $\underset{\nu_{j}<1}{O}$ & (9) & $\begin{array}{l}\text { iff } 2(i-1) \stackrel{(\dagger)}{=} m \\
\quad \& \mu_{3}=0\end{array}$ & 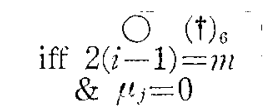 \\
\hline
\end{tabular}

which shows that, by looking at the asymptotic order of $f, g$ when $r$ o, we can know whether $\theta \in L^{2} A^{2}\left(C_{0, \varepsilon}(N)\right)$ or not.

By applying this way one after another, we get Figures 2.1, 2.2.

Figure 2.1.

$\mathrm{O}=$ "Yes". $\quad \mathrm{x}=$ "No". (O)="Yes \&=0". $\quad C_{0, s}=C_{0, s}(\mathrm{~N})$.

$(\dagger)_{1}$ : (O) iff $2 i \leqq m-1 \& \mu_{j}=0 . \quad(\dagger)_{1}:$ (ᄋ) iff $2 i \geqq m \div 3 \& \mu_{j}=0$.

$(\dagger)_{2}$ : and then (O).

$(\dagger)_{3}$ : and, then $(0$.

$(\dagger)_{3}$ : and, then $d \cdot=\delta \cdot=0$.

$(\dagger)_{6}:$ and, then $d \cdot=\delta \cdot:=0$. 


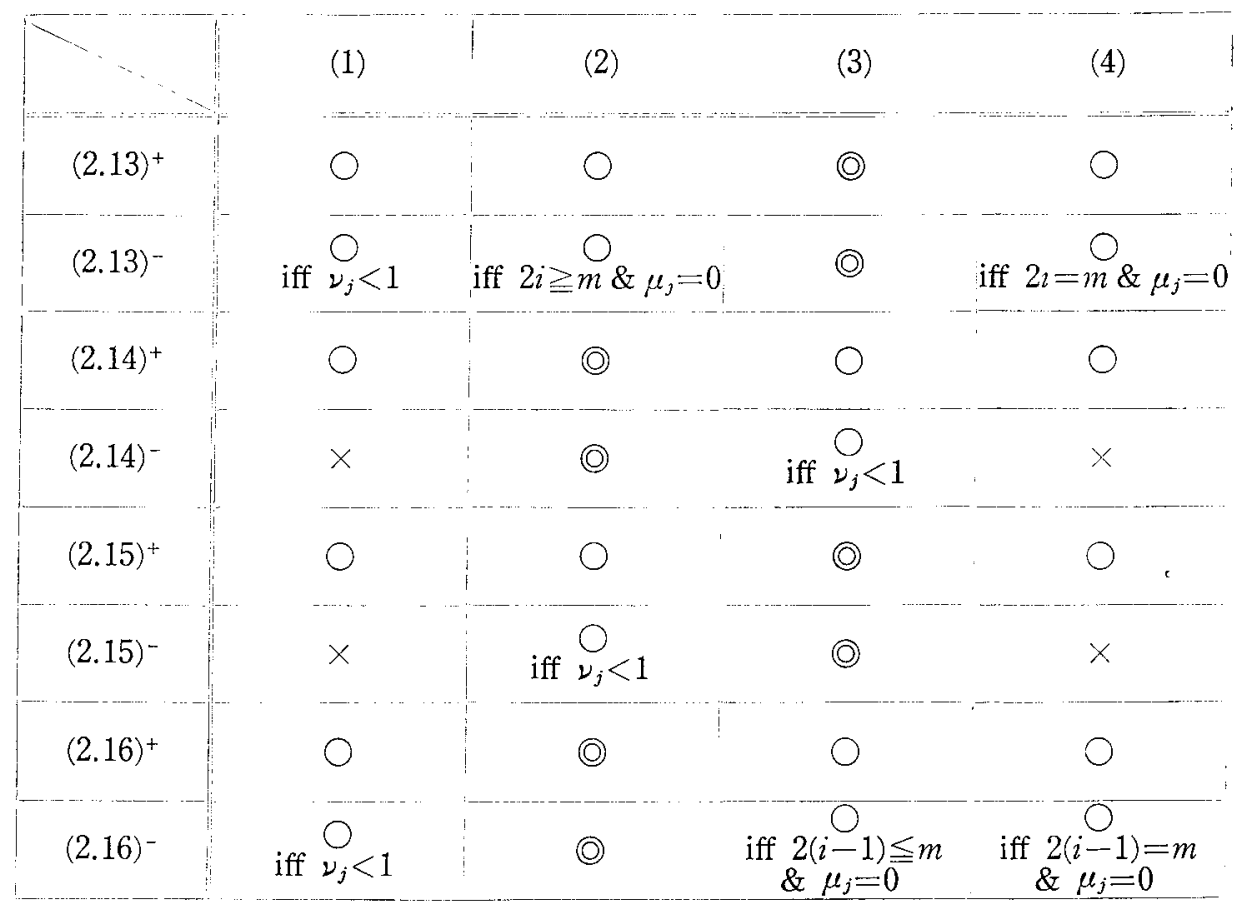

Figure 2.2: We continue to use the notations of Figure 2.1.

By the way, we remark, as a preparation for the following sections, that

$$
\|\theta\|_{(r, r)}^{2}=r^{m} \int_{N}|\theta|_{x}^{2} \approx 1_{\tilde{x}}, \quad x=(r, \tilde{x}),
$$

where $|\theta|_{x}$ is the pointwise norm defined by $(0.3)$.

Finally we will prepare for the study of the spectral representations of $\bar{J}_{b c, V, \imath}$ in Cases $\mathrm{B}(V), \mathrm{C}(V)$. The proof depends on the formula (2.23).

\section{LEMMA 2.3.}

(1) In Case B,

$$
\begin{aligned}
& \left\|(2.13)_{\tilde{\mathscr{H}}(k)}^{+}\right\|_{(r, r)}=O(1), \quad\left\|d(2.13)_{\tilde{\mathscr{H}}(k)}^{+}\right\|_{(r, r)}=O(1), \\
& \left\|(2.13)_{\overline{\mathscr{H}}(k)}^{-}\right\|_{(r, r)}=O(1), \quad\left\|d(2.13)_{\overline{\mathscr{H}}(k)}^{-}\right\|_{(r, r)}=O(1) .
\end{aligned}
$$

(2) In Case $C$,

$$
\left\|(2.16)_{\tilde{\mathscr{T}}(k)}^{+}\right\|_{(r, r)}=o(1), \quad\left\|\delta(2.16)_{\tilde{\mathscr{K}}(k)}^{+}\right\|_{(r, r)}=O(1),
$$




$$
\left\|(2.16)_{\overline{\mathscr{T}}(k)}^{-}\right\|_{(r, r)}=O(1), \quad\left\|\delta(2.16)_{\tilde{A}(k)}^{-}\right\|_{(r, r)}=o(1)
$$

Here $(2.13)_{\tilde{\mathscr{H}}(k)}^{ \pm}$etc. mean $r^{\alpha(i)} J_{ \pm \nu(\tilde{\mathscr{H}}(i))_{j}}(\lambda r) \omega_{\tilde{\mathscr{H}}(i),}$ etc.

\section{§3. Functional calculus on the metric cone.}

We continue to use the notations of $\S 2$. Let $\bar{J}_{2}$ be the Laplacian on the metric cone $C(N)$ defined by (1.29). Since it is self-adjoint, it has the spectral representation

$$
\bar{J}_{i}=\int \lambda d E(\lambda) .
$$

Hence, for any Borel measurable function $f$ on $\boldsymbol{R}$, the operator $f\left(\bar{A}_{t}\right)$ is defined by

$$
f\left(\bar{J}_{\imath}\right)=\int f(\lambda) d E(\lambda)
$$

In this section, we will try to describe the formal kernel $k_{f}$ of $f\left(\bar{U}_{\imath}\right)$ definitely. It is already described in the case where forms are of co-closed type ([4.1] of $[2])$, or in the case where $i=0([6])$. Here we will remove these restrictions, but the way of proof is similar to [6].

Unless confusions occur, $\nu(b c, \tilde{c c}(i))_{3}, \omega_{b c, \tilde{c}(i),,}$, etc. are abbreviated to $\nu(\tilde{c} \tilde{c}(i))_{,}$, $\omega_{c \tilde{c}(i), \jmath}$, etc., or more informally, to $\nu_{\jmath}$, $\omega_{j}$, etc. in certain circumstances.

Using the notation $x_{j}=\left(r_{j}, \tilde{x}_{j}\right) \in C(N)$, we set

$$
\begin{aligned}
& k_{f}\left(x_{1}, x_{2} ; \tilde{c c}(i)\right)=\sum_{j}\left(r_{1} r_{2}\right)^{\alpha(i)} \int_{0}^{\infty} f\left(\lambda^{2}\right) J_{\nu(\tilde{c}(i))_{j}}\left(\lambda r_{1}\right) J_{\nu(\tilde{c}(i))_{j}}\left(\lambda r_{2}\right) \lambda d \lambda \\
& \cdot \omega_{\tilde{c} \tilde{c}(i), j}\left(\tilde{x}_{1}\right) \otimes \omega_{c \tilde{c}(i), j}\left(\tilde{x}_{2}\right) \\
& =k_{f}\left(x_{1}, x_{2} ; \widetilde{\mathscr{H}}(i)\right)+k_{f}\left(x_{1}, x_{2} ; \tilde{c e}(i)\right), \\
& k_{f}\left(x_{1}, x_{2} ; d \tilde{c e}(i)+d r \wedge \tilde{c e}(i)\right) \\
& =\sum_{j} \frac{\left(r_{1} r_{2}\right)^{\alpha(i)}}{a^{+}(\tilde{c e}(i))_{j}-a^{-}(\tilde{c e}(i))_{j}} \\
& \times\left(\begin{array}{l}
\int_{0}^{\infty} f\left(\lambda^{2}\right) J_{\nu(\tilde{c} \tilde{e}(i))_{j-1}}\left(\lambda r_{1}\right) J_{\nu(\tilde{c} \tilde{e}(i))_{j-1}}\left(\lambda r_{2}\right) \lambda d \lambda \\
\int_{0}^{\infty} f\left(\lambda^{2}\right) J_{\nu(\tilde{c} \tilde{e}(i))_{j}+1}\left(\lambda r_{1}\right) J_{\nu(\tilde{c} \tilde{e}(i))_{j}+1}\left(\lambda r_{2}\right) \lambda d \lambda
\end{array}\right) \\
& \times\left\{\left(\begin{array}{c}
\frac{1}{a^{+}(\tilde{c e}(i))_{\jmath}} \\
-\frac{1}{a^{-}(\tilde{c} \tilde{e}(i))_{j}}
\end{array}\right) r_{1} r_{2} d \omega_{\tilde{c}(i), j}\left(\tilde{x}_{1}\right) \otimes d \omega_{\tilde{c}(i), j}\left(\tilde{x}_{2}\right)\right.
\end{aligned}
$$




$$
\begin{aligned}
& \div\left(\begin{array}{r}
1 \\
-1
\end{array}\right) r_{1} \tilde{d} \omega_{c \tilde{E}(i), j}\left(\tilde{x}_{1}\right) \otimes d r_{2} \wedge \omega_{c \tilde{E}(i), j}\left(\tilde{x}_{2}\right) \\
& +\left(\begin{array}{r}
1 \\
-1
\end{array}\right) r_{2} d r_{1} \wedge \omega_{\tilde{c}(i), j}\left(\tilde{x}_{1}\right) \otimes \tilde{d} \omega_{\tilde{c \varepsilon}(i), j}\left(\tilde{x}_{2}\right) \\
& \left.+\left(\begin{array}{c}
a^{+}(\tilde{c e}(i))_{2} \\
-a^{-}(\tilde{c} \tilde{e}(i))_{2}
\end{array}\right) d r_{1} \wedge \omega_{\tilde{c} \tilde{e}(i), j}\left(\tilde{x}_{1}\right) \otimes d r_{2} \wedge \omega_{c \tilde{e}(i), j}\left(\tilde{x}_{9}\right)\right\} \\
& =k_{f}(; d \tilde{c e}(i), d \tilde{c}(i))+k_{f}(; d \tilde{c}(i), d r \wedge \tilde{c}(i)) \\
& +k_{f}(; d r \wedge \tilde{c}(i), d \tilde{c}(i))+k_{f}(; d r \wedge \tilde{c}(i), d r \wedge \tilde{c}(i)) \\
& =k_{f}(; d \tilde{c e}(i), d \tilde{c e}(i)+d r \wedge \tilde{c e}(i))+k_{f}(; d r \wedge \tilde{c e}(i), d \tilde{c e}(i) \div d r \wedge \tilde{c e}(i)) \text {, } \\
& \text { (3.5) } \quad k_{f}\left(x_{1}, x_{2} ; d r \wedge \tilde{c}(i)\right)=\sum_{j}\left(r_{1} r_{3}\right)^{a(i)} \int_{0}^{\infty} f\left(\lambda^{2}\right) J_{2(\tilde{c}(i), \imath-1)_{j}}\left(\lambda r_{1}\right) J_{L(\tilde{c}(\imath), \imath-1)_{j}}\left(\lambda r_{2}\right) \lambda d \lambda \\
& \cdot d r_{1} \wedge \omega_{\tilde{c}(i), j}\left(\tilde{x}_{1}\right) \otimes d r_{2} \wedge \omega_{\check{c}(2), j}\left(\tilde{x}_{2}\right) \\
& =k_{f}(; d r \wedge \tilde{\mathscr{H}}(i))+k_{f}(; d r \wedge \tilde{e}(i)) .
\end{aligned}
$$

Moreover, for a given subspace $V(i) \subset \widetilde{\mathscr{H}}_{b c}^{i}(N)$, we take $\left\{\omega_{V(i), j}\right\}$, that is, an orthonormal basis of $V(i)$, and set

$$
\begin{gathered}
k_{f}\left(x_{1}, x_{2} ; V(i)\right)=\sum_{j}\left(r_{1} r_{2}\right)^{r(i)} \int_{0}^{\infty} f\left(\lambda^{2}\right) J_{ \pm 2\left(V^{\prime}(i)\right)_{j}}\left(\lambda r_{1}\right) J_{=2(V(i))_{j}}\left(\lambda r_{2}\right) \lambda d \lambda \\
\cdot \omega_{V(i), j}\left(\tilde{x}_{1}\right) \otimes \omega_{V(i), j}\left(\tilde{x}_{2}\right), \\
k_{f}\left(x_{1}, x_{2} ; d r \wedge V(i)\right)=\sum_{j}\left(r_{1} r_{2}\right)^{n(i)} \int_{0}^{\infty} f\left(\lambda^{2}\right) J_{=2(V(i), l-1)}\left(\lambda r_{1}\right) J_{\Sigma 2(V(i), 2-1)}\left(\lambda r_{2}\right) \lambda d \lambda \\
\cdot d r_{1} \wedge \omega_{V(i), j}\left(\tilde{x}_{1}\right) \otimes d r_{2} \wedge \omega_{V(i), j}\left(\tilde{x}_{2}\right),
\end{gathered}
$$

where $\nu(V(i))_{0}=\sqrt{ } 0+\alpha(i)^{2}=|\alpha(i)|, \nu(V(i), i-1){ }_{\jmath}=|\alpha(i-1)|$.

These expressions are easy to read, I think, but it is best to give some remarks. For example, in $(3.4)$, the terms $k_{f}(; d \tilde{c e}(i), d \tilde{c} \tilde{e}(i)), \cdots, k_{f}(; d r \wedge \tilde{c} \tilde{e}(i)$, $d r \wedge \tilde{c e}(i))$ denote the first, the second, the third and the fourth terms in the preceding expression respectively, i.e.,

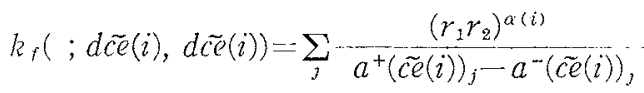

$$
\begin{aligned}
& \times\left(\begin{array}{l}
\int_{0}^{\infty} f\left(\lambda^{i}\right) J_{\nu(\tilde{c} \tilde{e}(i))_{j-1}}\left(\lambda r_{1}\right) J_{\nu(\tilde{c} \tilde{e}(i))_{j}{ }^{-1}}\left(\lambda r_{2}\right) \lambda d \lambda \\
\int_{0}^{\infty} f\left(\lambda^{2}\right) J_{\dot{*}(\tilde{c} \tilde{e}(i))_{j}+1}\left(\lambda r_{1}\right) J_{2(\tilde{c} \tilde{\varepsilon}(i))_{j+1}}\left(\lambda r_{2}\right) \lambda d \lambda
\end{array}\right) \\
& \times\left(\begin{array}{c}
\frac{1}{a+(\tilde{c e}(i))_{3}} \\
-\frac{1}{a-(\tilde{c}(i))_{3}}
\end{array}\right) r_{1} r_{2} \tilde{d} \omega_{\tilde{c} \tilde{\varepsilon}(i), j}\left(\tilde{x}_{1}\right) \otimes \tilde{d} \omega_{\tilde{c} \tilde{e}(i), j}\left(\tilde{x}_{2}\right),
\end{aligned}
$$


etc. Moreover the terms $k_{f}(; d \tilde{c e}(i), d \tilde{c e}(i)+d r \wedge \tilde{c e}(i))$ and $k_{f}(; d r \wedge \tilde{c e}(i), d \tilde{c e}(i)$ $+d r \wedge \tilde{c e}(i))$ denote " $k_{f}(; d \tilde{c e}(i), d \tilde{c} \tilde{e}(i))+k_{f}(; d \tilde{c e}(i), d r \wedge \tilde{c e}(i))$ " and " $k_{f}(; d r \wedge \tilde{c e}(i)$, $d \tilde{c e}(i))+k_{f}(; d r \wedge \tilde{c e}(i), d r \wedge \tilde{c e}(i)) "$.

The expressions of these types will appear sometimes in the following sections, in which we will not give the complete descriptions of them because they are completely analogous to $(3.3)-(3.7)$.

We can now state the main theorem of this section.

THEOREM 3.1. The formal kernel $k_{f}\left(x_{1}, x_{2} ; i\right)$ of $f\left(J_{2}\right)$ can be urrtten as follows in the distribution sense.

(1) In Case A,

$$
\begin{aligned}
k_{f}(; i)= & k_{f}(; \tilde{c c}(i))+k_{f}(; d \tilde{c e}(i-1)+d r \wedge \tilde{c e}(i-1)) \\
& +k_{f}(; d r \wedge \tilde{c}(i-1)) .
\end{aligned}
$$

(2) In Case $B(V(k))$,

$$
\begin{aligned}
k_{f}(; i)= & k_{f}^{\perp}(; V(k))+k_{f}\left(; V(k)^{\perp}\right)+k_{f}(; \tilde{c}(k)) \\
& +k_{f}(; d \tilde{c e}(k-1)+d r \wedge \tilde{c e}(k-1))+k_{j}(; d r \wedge \tilde{c}(k-1)) .
\end{aligned}
$$

(3) In Case $C(V(k))$,

$$
\begin{aligned}
k_{f}(; i)= & k_{f}(; \widetilde{c c}(k+1)) \div k_{f}(; d \tilde{c e}(k) \div d r \wedge \tilde{c e}(k)) \\
& +k_{f}(; d r \wedge V(k)) \div k_{f}^{-}\left(; d r \wedge V(k)^{!}\right)+k_{f}(; d r \wedge \tilde{e}(k)) .
\end{aligned}
$$

The proof is so long and complicated that we divide it into Steps 1 - III.

Step I. Steps II $-\mathrm{V}$ are devoted to the proof of (1). Steps IT, III are respectively devoted to the proofs of (2), (3).

According to the decomposition (2.6), we set

$$
\begin{aligned}
& L^{2} \Lambda^{i ; \tilde{c}}=\left\{\theta \in L^{2} \Lambda^{2}(C(N)) \mid \theta=\sum_{j} g_{c \tilde{c}(i), j} \omega_{c \tilde{c}(i), j}\right\},
\end{aligned}
$$

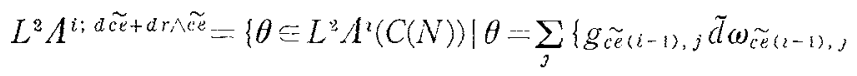

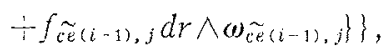

$$
\begin{aligned}
& L^{2} \Lambda^{i ;} d r \wedge \tilde{c}_{-}=\left\{\theta \in L^{2} \Lambda^{2}(C(N)) \mid \theta=\sum_{j} \int_{\tilde{c}(i-1), j} d r \wedge \omega_{\tilde{c}(t-1), j}\right\} .
\end{aligned}
$$

Then we have the orthogonal decomposition

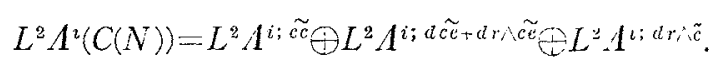

The restrictions of $\bar{J}_{\imath}$ to the intersections with dom $J_{\imath}$ and the three subspaces

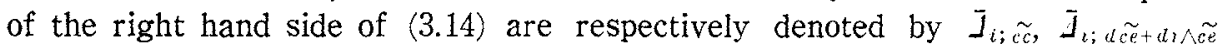
and $\bar{J}_{i ; a r \wedge \tilde{c}}$. Since we know from (2.7) that their respective ranges are contained in the respective subspaces of the right hand side of (3.14), it suffices to study 
their formal kernels, respectively.

Since the proof is based on the elementary properties of Hankel transform, we will list up some of them concerned to us.

If $g(r)$ is a smooth function on $\boldsymbol{R}^{+}$with compact support, its Hankel transform $H_{\nu}(g)$ is defined by

$$
H_{\nu}(g)=\int_{0}^{\infty} g(r) J_{\nu}(\lambda r) r d r
$$

The Hankel inversion formula ([14] III Page 149) says

$$
g(r)=H_{\nu}\left(H_{\nu}(g)\right)(r),
$$

with $\nu>-1$. Moreover we have the Plancherel formula

$$
\int_{0}^{\infty}|g(r)|^{2} r d r=\int_{0}^{\infty}\left|H_{\nu}(g)(\lambda)\right|^{2} \lambda d \lambda .
$$

Thus $H_{\nu}$ can be extended to the Hilbert space isomorphism

$$
L^{2}\left(\boldsymbol{R}^{+}, r d r\right) \cong L^{2}\left(\boldsymbol{R}^{+}, \lambda d \lambda\right) .
$$

Step II. The purpose of this step is to show that the formal kernel of $\bar{J}_{i ; \widetilde{c c}}$ in Case $\mathrm{A}$ is equal to $k_{f}(; \widetilde{c c}(i))$. In this step, we set $\bar{J}_{\imath}=\bar{J}_{i ; \tilde{c c}}, L^{2} \Lambda^{r}=$

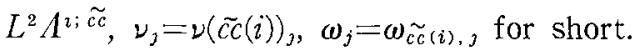

First, remark that, if we set

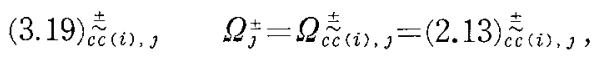

then, from Fig. $2.2, \Omega_{j}^{+}$belongs to dom $\bar{A}_{2}$ near the singular point.

Let's take $g_{j}(r) \omega_{j} \in L^{2} A^{\imath}$. Since (2.23) and (3.17) yield

$$
\left\|g_{j} \omega_{j}\right\|^{2}=\int_{0}^{\infty}\left(r^{-\alpha(\imath)} g_{\jmath}(r)\right)^{2} r d r=\int_{0}^{\infty}\left|H_{\nu}\left(r^{-\alpha(i)} g_{j}\right)(\lambda)\right|^{2} \lambda d \lambda,
$$

we have

$$
\begin{gathered}
r^{-\alpha(i)} g_{j}(r) \in L^{2}\left(\boldsymbol{R}^{+}, r d r\right), \\
\left\|g_{j} \omega_{j}\right\|=\left\|H_{\nu}\left(r^{-\alpha(\imath)} g_{\jmath}\right)\right\|_{L^{2}\left(\boldsymbol{R}^{+}, \lambda d \lambda\right)} .
\end{gathered}
$$

Hence the map $\sum_{j} g_{j} \omega_{j} \mapsto H_{\nu_{j}}\left(r^{-\alpha(i)} g_{j}\right)$ induces

$$
\mathscr{H}_{2,}: L^{2} A^{2} \rightarrow L^{2}\left(\boldsymbol{R}^{+}, \lambda d \lambda\right)
$$

Moreover we have

$$
\mathscr{H}_{\nu},\left(\sum_{j} g_{j} \omega_{j}\right)=\left\langle g_{j} \omega_{j}, \Omega_{j}^{+}\right\rangle_{C(N)} .
$$

By this formula, we can show that, for $g_{j} \omega_{j} \in \operatorname{dom} \bar{J}_{\imath}$, 


$$
\mathscr{K}_{\nu_{j}}\left(\mathcal{S}\left(g_{j} \omega_{j}\right)\right)=\lambda^{2} \mathscr{H}_{\nu_{j}}\left(g_{j} \omega_{j}\right) .
$$

In fact, since there exists a sequence $\left\{h_{k}(r) \omega_{j}\right\}_{k} \subset$ dom $J_{\imath}$ such that $h_{k}(r)=0$ for $r$ large, and $h_{k} \omega_{j} \rightarrow g_{j} \omega_{j}, d\left(h_{k} \omega_{j}\right) \rightarrow d\left(g_{j} \omega_{j}\right), \delta\left(h_{k} \omega_{j}\right) \rightarrow \delta\left(g_{j} \omega_{j}\right), \quad \Delta\left(h_{k} \omega_{j}\right) \rightarrow \Delta\left(g_{j} \omega_{j}\right)$ in the $L^{2}$-sense when $k \rightarrow \infty$, it suffices to prove (3.25) for a form $g_{j} \omega_{j} \equiv$ dom $\bar{A}_{\imath}$ with $g_{j}(r)=0$ for $r$ large. And this is an immediate consequence of the formula (3.24) and the property of $\Omega_{j}^{+}$, that is, $\Delta \Omega_{j}^{+}=\lambda^{2} \Omega_{j}^{+}$.

Further, for a form $\theta=\sum g_{j} \omega_{j} \in \operatorname{dom} \bar{\Delta}_{\imath}$, we have $g_{j} \omega_{j} \equiv \operatorname{dom} I_{\imath}$ and $\Delta \theta=$ $\sum \Delta\left(g_{j} \omega_{j}\right)$.

Thus, letting $L^{2}\left(\boldsymbol{R}^{+}, \lambda d \lambda, l^{2}\right)$ be the Hilbert space consisting of $l^{2}$-valued square-integrable functions on $\boldsymbol{R}^{+}$with measure $\lambda d \lambda$ and define

$$
\mathscr{H}_{\nu(\tilde{c} \tilde{c}(i))}\left(\sum_{j} g, \omega_{j}\right)=\left\{\mathscr{H}_{\nu}\left(g_{j} \omega_{j}\right)\right\}
$$

for $\sum_{j} g_{,} \omega_{j} \in L^{2} \Lambda^{2}$, we get

LEMMA 3.2. We have the Hilbert space isomorphism

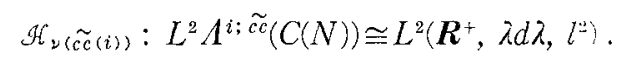

In particular, if $\theta \in \operatorname{dom} \bar{J}_{2} ; \tilde{c c}$, then

$$
\mathscr{H}_{\nu(\tilde{c} \tilde{c}(i))}(\Delta \theta)=\lambda^{2} \mathscr{K}_{\nu(\tilde{c} \tilde{c}(i))}(\theta) .
$$

In other words, (3.27) and (3.28) provide the spectral representation of $\bar{J}_{i ; \tilde{c} \text {. }}$ Hence the operation with $f\left(\bar{J}_{i, \tilde{c})}\right)$ on $\theta=\sum g_{j} \omega_{j} \in \operatorname{dom} f\left(\bar{J}_{i ;}, \tilde{c}\right)$ can be written as follows;

$$
\begin{aligned}
& f\left(\bar{J}_{1 ;} ; \tilde{c c}\right) \theta\left(r_{1}, \tilde{x}_{1}\right)=\mathscr{H}_{\nu(\tilde{c c}(2))}^{-1}\left(f\left(\lambda^{2}\right) \mathscr{M}_{\nu}(\tilde{\tilde{c}(1)}),(\theta)\right)\left(r_{1}, \tilde{x}_{1}\right) \\
& =\sum_{j} r_{1}^{\alpha(2)} \int_{0}^{\infty} f\left(\lambda^{2}\right) J_{\nu_{j}}\left(\lambda r_{1}\right)\left[\int_{0}^{\infty} r_{2}^{-\alpha(i)} g_{j}\left(r_{2}\right) J_{\nu_{j}}\left(\lambda r_{2}\right) r_{2} d r_{2}\right] \lambda d \lambda \omega_{j}\left(\tilde{x}_{1}\right) \\
& =\sum \int_{0}^{\infty}\left(r_{1} r_{2}\right)^{\alpha(\imath)}\left[\int_{0}^{\infty} f\left(\lambda^{2}\right) J_{\nu_{j}}\left(\lambda r_{1}\right) J_{\nu}\left(\lambda r_{2}\right) \lambda d \lambda\right] g_{j}\left(r_{2}\right) r_{2}^{n-\nu_{i}} d r_{2} \omega,\left(\tilde{x}_{1}\right) .
\end{aligned}
$$

Here the last expression is of the distribution sense.

Thus our purpose of this step was accomplished.

Step III. The purpose of this step is to show that the formal kernel of $f\left(\bar{J}_{i} ; d r \wedge \tilde{c}\right)$ in Case $\mathrm{A}$ is equal to $k_{f}(; d r \wedge \tilde{c}(i-1))$.

The proof is similar to Step II. Set

$$
\Omega_{\jmath}^{ \pm}=\Omega_{d r \wedge \tilde{c}(L-1)}^{ \pm}=(2.16)_{\tilde{c}(i-1), \jmath}^{ \pm} .
$$

Then, $\Omega_{j}^{+}$belongs to $L^{2} A^{2, d r \wedge \tilde{c}}$ near the singular point. Similar to $(3.23)$, the $\operatorname{map} \sum_{j} f_{j}(r) d r \wedge \omega_{\tilde{c}(i-1), j^{\mapsto}} \rightarrow H_{\nu(\tilde{c}(i-1), 2-2)}\left(r^{-r(i-1)} f_{j}\right)$ induces

$$
\mathscr{T}_{2(\tilde{c}(i-1), 2-2),}: L^{2} \lambda^{2 ; i r \wedge \tilde{c}} \rightarrow L^{2}\left(\boldsymbol{R}^{+}, \lambda d \lambda\right),
$$


and further we have

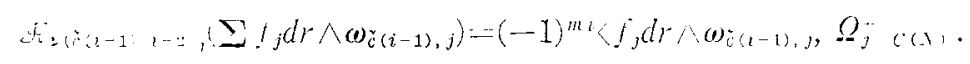

Hence, by the argument similar to Lemma 3.2 , we get

LEMMA 3.3. We have the Hibert space isomorphism

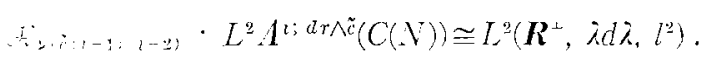

In particular, if $\theta=\mathrm{dom} J_{\ell, d r} \times$, then

$$
\therefore=(i(i-1), 2-2)(\Delta \theta)=\lambda^{2} \mathscr{G}_{i}(\tilde{c}(i-1), 1-\cdots),(\theta) .
$$

Hence our purpose is also accomplished by the argument similar to the one following Lemma 3.2 .

Finally remark that, if we insert " $b c$ " or " $a d b c$ " into the $\nu(\cdot)$ in order to indicate the boundary condition under consideration, we have

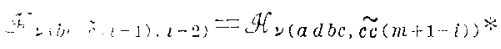

$$
\begin{aligned}
& \left.k_{f^{+1}} ; b c \text { dr } \wedge \tilde{c}(i-1)\right)=*^{-1} k_{f}(; a d b c, \tilde{c}(m--1-i)) *
\end{aligned}
$$

Step II. The purpose of this step is to show that the formal hernel of

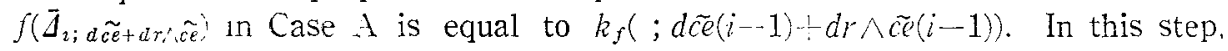

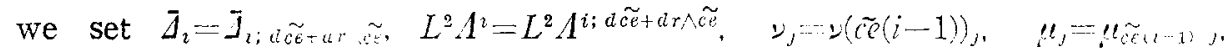

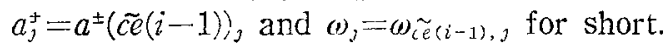

Here we cannot expect $(2.14)^{+}$and $(2.15)^{-}$to play the roles similar to (3.19)and $(3.30)^{+}$. Let's start with constructing the $i$-forms from $(2.14)^{+}$and $(2.15)^{-}$, which are suitable for our purpose.

The following formulas for Bessel functions are well known ([14] III Pages $158,159)$;

$$
\begin{aligned}
& J_{j}(\lambda r)=\frac{\lambda r}{2 \nu_{j}}\left\{J_{\nu_{j-j}}(\lambda r)+J_{i_{j-1}}(\lambda r)\right\}, \\
& J_{i j}^{\prime}(\lambda r)=\frac{1}{2}\left\{J_{\nu_{j-1}}(\lambda r)-J_{\nu_{j+1}+1}(\lambda r)\right\} .
\end{aligned}
$$

By applying $\left(3.36,(2.14)^{-}\right.$can be rewritten as follows (see $\left.\left(2.14^{\prime}\right)^{-}\right) \cdot$

$$
\begin{aligned}
& \frac{\lambda}{22}, \cdots\left({ }^{2},\left\{J_{2,-1}(\lambda r)-J_{\nu_{j}+1}(\lambda r)\right\} d \omega,\right. \\
& \frac{\lambda}{2 y_{j}} r^{\mu(u-1)}\left\{a_{j}^{+} J_{\nu_{j-1}}(\lambda r) \div a_{j} J_{\nu_{2}-1}(\lambda r)\right\} d r \wedge \omega_{j} .
\end{aligned}
$$

Similarly, $\left(2.15^{-}\right.$multiplied by $(-1)^{m(i-1)+1}$ can be rewritten as follows see $\left.\left(2.15^{\prime}\right)^{+}\right)$: 


$$
\begin{aligned}
& -\frac{\lambda}{2 \nu_{3}} r^{n(i)}\left\{a_{j}^{-} J_{\nu_{3}-1}(\lambda r)+a_{j}^{+} J_{\nu_{3} ; 3}(\lambda r)\right\} \tilde{d} \omega_{3}
\end{aligned}
$$

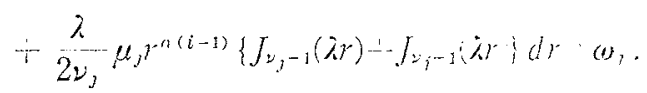

Hence, if we set

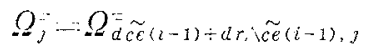

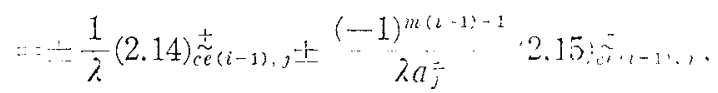

then

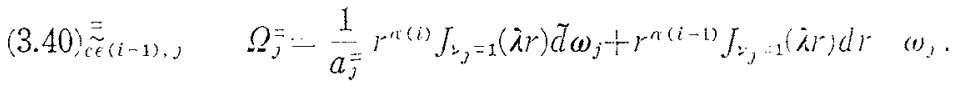

These $i$-forms play the roles similar to $(3.19)^{+}$or $(3.30)^{-}$.

First, from Fig. $2.2, \Omega \neq$ belongs to dom $\bar{J}_{2}$ near the singular pont. Moreover, from (2.23) and (3.17), if $g_{j} \tilde{d} \omega_{j} \div f_{j} d r \wedge \omega_{j} \in L^{2} A^{2}$, we have

$$
\begin{aligned}
& \left\|g_{j} \tilde{d} \omega_{3}+f_{j} d r \wedge \omega_{j}\right\|^{2}=\mu \int_{j}^{\infty}\left(r^{-r(i)} g_{3}(r)\right)^{2} r d r-\int_{0}^{\infty}\left(r^{\cdots} \cdots r, r \cdot r d r,\right.
\end{aligned}
$$

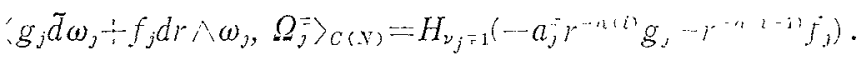

Moreover, similarly to the proof of (3.25), if $g_{J} J \omega_{3}-f_{J} d r, \omega_{j}=\operatorname{dom} I_{2}$,

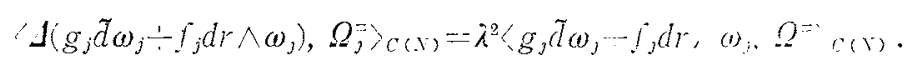

LEMMA 3.4. The nap

$$
\begin{aligned}
& \sum_{j}\left(g_{j} \tilde{d} \omega_{j} \div f_{j} d r \wedge \omega_{j}\right) \\
& \rightarrow\left\{\left(-a_{3}^{-j} r^{-a(i)} g_{j} \div r^{-\alpha(i-1)} f_{3},-a_{3}^{+} r^{-\cdots(i)}+r^{\cdots}+i^{\prime} i_{3}\right\}\right.
\end{aligned}
$$

induces the Banach space isomorphism

$$
L^{2} A^{i ; a \tilde{e}+d r, \tilde{e}}(C(N)) \cong L^{2}\left(\boldsymbol{R}^{+}, \operatorname{rdr}, l^{2} I l^{*}\right) .
$$

Proof.

the norm of the r.h.s. of $(3.44)\}^{2}$

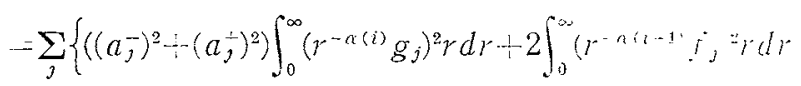

$$
\begin{aligned}
& -2 \alpha(i-1) \int_{0}^{\infty}\left(r^{-\alpha(i)} g_{j}\right)\left(r^{-a(i-1)} j_{j}\right) r d r_{j} \\
& \geq \sum\left\{\left(4 \alpha(i-1)^{2}+2 \mu_{j}\right) \int_{0}^{\infty}\left(r^{-\alpha(i)} g_{j}\right)^{2} r d r+2 \int_{0}^{\infty}\left(r+r_{j}+r d r\right.\right.
\end{aligned}
$$


404

MASAYOSHI NAGASE

$$
\begin{aligned}
& \left.\quad-\alpha(l-1)^{2} \int_{0}^{\infty}\left(r^{-\alpha(i)} g_{j}\right)^{2} r d r-\int_{0}^{\infty}\left(r^{-\alpha(i-1)} f_{j}\right)^{2} r d r\right\} \\
& =\sum_{j}\left\{\left(3 \alpha(i-1)^{2}+2 \mu_{j}\right) \int_{0}^{\infty}\left(r^{-\alpha(i)} g_{j}\right)^{2} r d r+\int_{0}^{\infty}\left(r^{-\alpha(i-1)} f_{j}\right)^{2} r d r\right\} \\
& \geqq\{\text { the norm of the l.h.s. of }(3.44)\}^{2} \text {. } \\
& \text { \{the norm of the } r . h . s . \text { of }(3.44)\}^{2} \\
& \leqq \sum_{j}\left\{\left(5 \alpha(i-1)^{2}+2 \mu_{j}\right) \int_{0}^{\infty}\left(r^{-\alpha(i)} g_{j}\right)^{2} r d r+3 \int_{0}^{\infty}\left(r^{-\alpha(i-1)} f_{j}\right)^{2} r d r\right\} \\
& \leqq K\{\text { the norm of the l.h.s. of }(3.44)\}^{2} .
\end{aligned}
$$

Finally, if we set, for $\left\{\left(F_{j}(r), G(r)\right)\right\}_{j} \in L^{2}\left(\boldsymbol{R}^{+}, r d r, l^{2} \oplus l^{2}\right)$,

$$
\begin{aligned}
& g_{j}(r)=\frac{r^{\alpha(i)}}{a_{j}^{+}-a_{j}^{-}}-\left\{F_{j}(r)-G_{j}(r)\right\}, \\
& f_{j}(r)=\frac{r^{\alpha(i-1)}}{a_{j}^{+}-a_{j}^{-}}-\left\{a_{j}^{+} F_{j}(r)-a_{j}^{-} G_{j}(r)\right\},
\end{aligned}
$$

then $\Sigma\left(g_{j} d \omega_{j} \div f_{j} d r \wedge \omega_{j}\right) \subseteq L^{2} A^{2}$ and, moreover, its image by the map (3.44) is $\left\{\left(F_{j}(r), G_{j}(r)\right)\right\}_{\jmath}$.

On the other hand, the map

$$
\left\{\left(F_{j}(r), G_{j}(r)\right)\right\}_{j} \mapsto\left\{\left(H_{\nu_{j}-1}\left(F_{j}\right), H_{\nu_{j}+1}\left(G_{j}\right)\right\}_{j}\right.
$$

induces the Hilbert space isomorphism

$$
L^{2}\left(\boldsymbol{R}^{\leftarrow}, r d r, l^{2} \oplus l^{2}\right) \cong L^{2}\left(\boldsymbol{R}^{+}, \lambda d \lambda, l^{2} \oplus l^{2}\right) .
$$

Hence, by composing the maps (3.45) and (3.48), we get

LEMMA 3.5. We have the Banach space isomorphism

$$
\mathscr{H}_{2(a \tilde{c} \tilde{c}: 1-1 ;-d r, \tilde{e}(i-1))}: L^{2} \Lambda^{i ; d \tilde{c e}+d r \wedge \tilde{e}}(C(N)) \cong L^{2}\left(\boldsymbol{R}^{+}, \lambda d \lambda, l^{2} \bigoplus l^{2}\right) .
$$

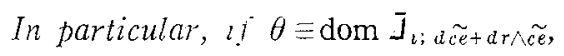

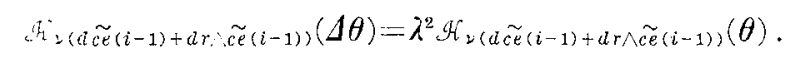

(3.50) is an immediate consequence of (3.42) and (3.43).

We can now accomplish our purpose. Let's take $\theta=\Sigma\left(g_{j} \tilde{d} \omega_{j}+f_{j} d r \wedge \omega_{j}\right) \in$ $\operatorname{dom} f\left(\bar{J}_{i ; d \tilde{e}+d r ; \tilde{c})}\right)$ and consider $f\left(\bar{J}_{i} ; d \tilde{c e}+d r \wedge \tilde{\varepsilon}\right) \theta\left(r_{1}, \tilde{x}_{1}\right)$. By $(3.46)$, the coefficient of $\tilde{d} \omega_{j}\left(\tilde{x}_{1}\right)$ is

$$
\begin{aligned}
\left(a_{j}^{--}-a_{j}^{-}\right)^{-1} \int_{0}^{\infty}\left(r_{1} r_{2}\right)^{\alpha(i)}\left[\int _ { 0 } ^ { \infty } f ( \lambda ^ { 2 } ) \left\{-a_{j}^{-} J_{\nu_{j}-1}\left(\lambda r_{1}\right) J_{\nu_{j}-1}\left(\lambda r_{2}\right)\right.\right. & \\
& \left.\left.+a_{j}^{+} J_{\nu_{j}+1}\left(\lambda r_{1}\right) J_{\nu_{j}+1}\left(\lambda r_{2}\right)\right\} \lambda d \lambda\right] g_{j}\left(r_{2}\right) r_{2}^{m-22} d r_{2}
\end{aligned}
$$




$$
\begin{aligned}
+\left(a_{j}^{+}-a_{j}^{-}\right)^{-1} \int_{0}^{\infty} r_{1}^{\alpha(i)} r_{2}^{\alpha(i-1)} & {\left[\int _ { 0 } ^ { \infty } f ( \lambda ^ { 2 } ) \left\{J_{\nu_{j-1}-1}\left(\lambda r_{1}\right) J_{\nu_{j-1}}\left(\lambda r_{2}\right)\right.\right.} \\
& \left.\left.-J_{\nu_{j}+1}\left(\lambda r_{1}\right) J_{\nu_{j}+1}\left(\lambda r_{2}\right)\right\} \lambda d \lambda\right] f_{j}\left(r_{2}\right) r_{2}^{m-2(i-1)} d r_{2}
\end{aligned}
$$

Hence, those terms of the formal kernel of $f\left(\bar{J}_{i ; d \tilde{c e}+d r \wedge \tilde{c e})}\right.$ which involve the $d \omega_{j}\left(\tilde{x}_{1}\right) \otimes d \omega_{j}\left(\tilde{x}_{2}\right)$ 's and the $d \omega_{j}\left(\tilde{x}_{1}\right) \otimes d r_{2} \wedge \omega_{j}\left(\tilde{x}_{2}\right)$ 's are obviously $k_{f}(; d \tilde{c e}(i-1)$, $d \tilde{c e}(i-1))$ and $k_{f}(; d \tilde{c}(i-1), d r \wedge \tilde{c} \tilde{e}(i-1))$, respectively. Next, similarly, by (3.46), the coefficient of $d r \wedge \omega_{j}\left(\tilde{x}_{1}\right)$ is

$$
\begin{aligned}
&\left(a_{j}^{+}-a_{j}^{-}\right)^{-1} \int_{0}^{\infty} r_{1}^{\alpha(i-1)} r_{2}^{\alpha(i)} \mu_{j}\left[\int _ { 0 } ^ { \infty } f ( \lambda ^ { 2 } ) \left\{J_{\nu^{-1}}\left(\lambda r_{1}\right) J_{\nu_{j}-1}\left(\lambda r_{2}\right)\right.\right. \\
&\left.\left.-J_{\nu_{j}+1}\left(\lambda r_{1}\right) J_{\nu_{j}+1}\left(\lambda r_{2}\right)\right\} \lambda d \lambda\right] g_{j}\left\langle r_{2}\right) r_{2}^{m-22} d r_{2} \\
&+\left(a_{j}^{+}-a_{j}^{-}\right)^{-1} \int_{0}^{\infty}\left(r_{1} r_{2}\right)^{\alpha(i-1)}\left[\int _ { 0 } ^ { \infty } f ( \lambda ^ { 2 } ) \left\{a_{j}^{+} J_{\nu_{j}-1}\left(\lambda r_{1}\right) J_{\nu_{j-1}}\left(\lambda r_{2}\right)\right.\right. \\
&- \\
&\left.\left.-a_{j}^{-} J_{\nu_{j}+1}\left(\lambda r_{1}\right) J_{\nu_{j}+1}\left(\lambda r_{2}\right)\right\} \lambda d \lambda\right] f_{j}\left(r_{2}\right) r_{2}^{m-2(i-1)} d r_{2} .
\end{aligned}
$$

Hence those terms of the formal kernel of $f\left(\bar{A}_{i ; d \tilde{c e}+d r \wedge \tilde{c})}\right)$ which involve the $d r_{1} \wedge \omega_{j}\left(\tilde{x}_{1}\right) \otimes d \omega_{j}\left(\tilde{x}_{2}\right)^{\prime}$ s and the $d r_{1} \wedge \omega_{j}\left(\tilde{x}_{1}\right) \otimes d r_{2} \wedge \omega_{j}\left(\tilde{x}_{2}\right)$ 's are obviously $k_{f}(; d r \wedge \tilde{c}(i-1), d \tilde{c}(i-1))$ and $k_{f}(; d r \wedge \tilde{c e}(i-1), d r \wedge \tilde{c}(i-1))$, respectively.

Thus the purpose of this step was accomplished.

Step $V$. The proof of Theorem $3.1(1)$ is thus accomplished.

Step VI. Next, we will treat Case $\mathrm{B}(V(k))$. Pay attention to $(3.19)^{ \pm},(3.30)^{ \pm}$ and $(3.40)^{ \pm}$. In Case $\mathrm{A},(3.19)^{+},(3.30)^{+}$and $(3.40)^{ \pm}$belong to dom $\vec{A}_{2}$ near the singular point, which played the important roles in the proof of Theorem 3.1 (1). However, in Case $\mathrm{B}(V(k))$, the situations are slightly different because of the existence of the ideal boundary condition.

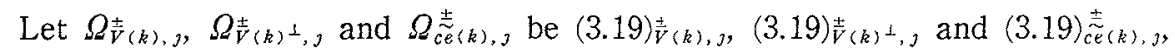
respectively. Observing Lemma 2.3 (1) and Fig. 2.2, we know

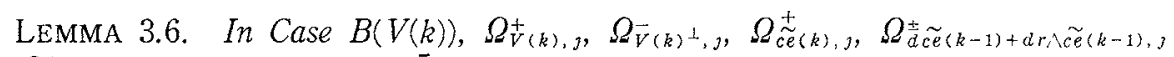
and $\Omega_{d r \wedge \tilde{c}(k-1), 0}^{+}$belong to dom $\bar{A}_{2}$ near the singular point.

Hence, for proving (3.9), Steps III, IV are still valid and Step II becomes valid by replacing $\Omega_{V(k)^{\perp}, \text { by }}^{+} \Omega_{\bar{V}(k)^{\perp}, \jmath}$.

Thus we may say that the proof of Theorem 3.1 (2) has been completed.

Step VII. Finally we will treat Case $\mathrm{C}(V(k))$. The situations are similar to Case $\mathrm{B}(V(k))$.

Let $\Omega_{d r \wedge V(k), \jmath}^{ \pm}, \Omega_{d r \wedge V(k)}^{ \pm}, j$ and $\Omega_{d r \wedge \tilde{e}(k), j}^{ \pm}$be $(3.30)_{V(k), \jmath}^{ \pm}, \quad(3.30)_{V(k)}^{ \pm}$, , and $(3.30)_{\tilde{e}(k), 3}^{ \pm}$respectively. Observing Lemma 2.3 (2) and Fig. 2.2, we get

LEMMA 3.7. In Case $\mathrm{C}(V(k)), \Omega_{\tilde{c}(k+1), j}^{+}, \Omega_{d \tilde{d} \tilde{e}(k)+\operatorname{dr} \wedge \tilde{c}(k), y}^{+}, \Omega_{\vec{d} r \wedge V(k),,}$, $\Omega_{a r \wedge V(k) \perp, j}^{+}$and $\Omega_{d r \wedge \tilde{e}(k),}^{+}$belong to dom $\bar{J}_{\imath}$ near the singular point. 
Hence, for proving (3.10), Steps II, IV are valid and Step III becomes valid by replacing $\Omega_{d r \wedge V(k), j}^{\perp}$ by $\Omega_{\bar{d} r \wedge V(k), \jmath}^{-}$.

Thus we may say that the proof of Theorem 3.1 (3) has been completed.

Thus the proof of Theorem 3.1 is complete. $f\left(\bar{J}_{2}\right)$.

Next we will make some remarks for applying Theorem 3.1 to the study of

First observe $k_{f}(; \tilde{c c}(i))$. It can be regarded as a family of functions of $\nu(\tilde{c}(i))=\sqrt{P_{\tilde{c} \tilde{c}} \tilde{A}_{i}+\alpha(i)^{2}}$ parametrized by $r_{1}, r_{2}$. More precisely, by the natural identification

$$
L^{2} A^{i: \tilde{c}}(C(N)) \cong L^{2}\left(\boldsymbol{R}^{+}, r^{m-2 v} d r, L^{2} A^{i ; \tilde{c}}(N)\right)
$$

with

$$
L^{2} \Lambda^{1, \widetilde{c}}(N)=\widetilde{\mathscr{H}}_{b c}^{i}(N) \oplus \overline{\tilde{\tilde{j}} \Lambda_{b c}^{i+1}(N)}
$$

the operator $k_{f}(; \tilde{c}(i))$ can be regarded as a linear operator

$$
L^{2}\left(\boldsymbol{R}^{+}, r^{m-\underline{2} \imath} d r, L^{2} A^{i, \tilde{c}}(N)\right) \rightarrow L^{2}\left(\boldsymbol{R}^{+}, r^{m-2 t} d r, L^{2} A^{i ; \tilde{c}}(N)\right) .
$$

From this view-point, we can apply the functional calculus for functions of $\nu(\tilde{c c}(i))$. Really this gives the crucial view-point; [6] provides the best example.

By the way, we wish to observe also (3.4)--(3.7) from the view-point similar to (3.55). However, what is corresponding to (3.53) is complicated. Hence it will be best to manage to avoid using such complicated ones.

Therefore, let's replace $\mu_{j}^{-1 / 2} r_{1} d \omega_{j}\left(\tilde{x}_{1}\right), \mu_{j}^{-1 / 2} r_{2} d \omega_{j}\left(\tilde{x}_{2}\right), d r_{1} \wedge \omega_{j}\left(\tilde{x}_{1}\right), d r_{2} \wedge \omega_{j}\left(\tilde{x}_{2}\right)$ by $\omega_{3}\left(\tilde{x}_{1}\right), \omega_{3}\left(\tilde{x}_{2}\right), \omega_{j}\left(\tilde{x}_{1}\right), \omega_{j}\left(\tilde{x}_{2}\right)$ in $(3.3)-(3.7)$. Here, remark that the subscripts " $\widetilde{c c}(i), \widetilde{c e}(i)$, etc." are omitted. Let $\tilde{k}_{f}(; \widetilde{c c}(i)), \tilde{k}_{f}(; d \tilde{c e}(i)+d r \wedge \widetilde{c e}(i)), \cdots, \tilde{k}_{f}^{ \pm}(; d r$ $\wedge V(i))$ be the operators constructed in the above way. Of course, $\tilde{k}_{f}(; \tilde{c} c(i))=$ $k_{f}(; \tilde{c c}(i))$. They can be regarded as linear operators similar to (3.55). Moreover, through the Hilbert space isomorphisms

with

$$
\begin{aligned}
& L^{2}\left(\boldsymbol{R}^{+}, r^{2 n-21} d r, d r \wedge L^{2} A^{2}(N)\right) \cong L^{2}\left(\boldsymbol{R}^{+}, r^{m-22} d r, L^{2} A^{2}(N)\right), \\
& \text { U) } U \\
& \sum f_{j} d r \wedge \omega_{3} \quad \longrightarrow \longrightarrow \sum f_{j} \omega_{j} \\
& L^{2}\left(\boldsymbol{R}^{+}, r^{m-2 \imath} d r, L^{2} A^{i ; \widetilde{c}}(N)\right) \cong L^{2}\left(\boldsymbol{R}^{+}, r^{m-2(i+1)} d r, L^{2} A^{i ; \tilde{e}}(N)\right) \text {, } \\
& \text { U) } U \\
& \sum g_{j} \omega_{\tilde{c} \tilde{e}(2), j} \longleftrightarrow \sum g_{3} \mu_{\tilde{c e}(i), j}^{-1 / 2} r \tilde{d} \omega_{\tilde{c e}(i),,}
\end{aligned}
$$

$$
\begin{aligned}
& L^{2} A^{2: \tilde{c}}(N)=\overline{\tilde{\tilde{o}} A_{b c}^{2+1}(N)}, \\
& L^{2} A^{i ; \tilde{e}}(N)=\overline{\tilde{d} A_{b c}^{2-1}(N),}
\end{aligned}
$$

they can be identified with the original ones, that is, $(3.3)-(3.7)$.

Moreover, if we set 


$$
\begin{aligned}
& \tilde{k_{f}}(; \tilde{c e}(i))=\left(r_{1} r_{2}\right)^{x(i)} \sum_{\jmath}\left\{\int_{0}^{\infty} f\left(\lambda^{2}\right) J_{\nu,-1}\left(\lambda r_{1}\right) J_{\nu,-1}\left(\lambda r_{2}\right) \lambda d \lambda\right. \\
& \left.+\int_{0}^{\infty} f\left(\lambda^{2}\right) J_{\nu_{j}+1}\left(\lambda r_{1}\right) J_{\nu_{j+1}}\left(\lambda r_{2}\right) \lambda d \lambda\right\} \omega_{j}\left(\tilde{x}_{1}\right) \otimes \omega_{j}\left(\tilde{x}_{2}\right), \\
& \tilde{k}_{f}(; \tilde{c}(i))==\left(r_{1} r_{2}\right)^{a(i)} \sum_{j} \frac{1}{2 \nu_{j}}\left\{\int_{0}^{\infty} f\left(\lambda^{2}\right) J_{\nu_{j-1}}\left(\lambda r_{1}\right) J_{\nu,-1}\left(\lambda r_{2}\right) \lambda d \lambda\right. \\
& \left.-\int_{0}^{\infty} f\left(\lambda^{2}\right) J_{\nu^{j+1}}\left(\lambda r_{1}\right) J_{\nu_{j+1}+1}\left(\lambda r_{2}\right) \lambda d \lambda\right\} \omega_{j}\left(\tilde{x}_{1}\right) \otimes \omega_{1}\left(\tilde{x}_{0}\right),
\end{aligned}
$$

with $\nu_{3}=\nu(\tilde{c} \tilde{e}(i))_{3}, \omega_{3}=\omega_{\tilde{c} \tilde{e}(i),}$, then we have

$$
\tilde{k}_{f}(; d \tilde{c e}(i), d \tilde{c}(i))=\frac{1}{2} \tilde{k}_{f}^{+}(; \tilde{c e}(i))-\alpha(i) \tilde{k_{f}}(; \tilde{c e}(i)),
$$

(3.62) $\quad \tilde{k}_{f}(; d \tilde{c e}(i), d r \wedge \tilde{c e}(i))==\tilde{k}_{f}(; d r \wedge \tilde{c e}(i), d \tilde{c e}(i))==\left(P_{c \tilde{e}} \tilde{d}_{\imath}\right)^{1 / 2} \tilde{k}_{\tilde{f}}(; \tilde{c}(i))$,

$$
\tilde{k}_{f}(; d r \wedge \tilde{c} \tilde{e}(i), d r \wedge \tilde{e}(i))=\frac{1}{2} \tilde{k}_{f}^{+}(; \tilde{e}(i))-\alpha(i) \tilde{k}_{\tilde{f}}(; \tilde{c}(i)) \text {. }
$$

Moreover, since

$$
\tilde{k}_{f}(; d r \wedge \tilde{c}(i))=\left(\frac{r_{2}}{r_{1}}\right)^{m-22} \tilde{*}^{-1} \tilde{k}_{f}(; a d b c, \tilde{c}(m-i)) \widetilde{*}
$$

and, in Cases $\mathrm{B}(V(k)), \mathrm{C}(V(k))$,

$$
\begin{aligned}
\tilde{k_{\bar{r}}}(; V(k))=\left(r_{1} r_{2}\right)^{1 / 2} \int_{0}^{\infty} f\left(\lambda^{2}\right) J_{-1 / 2}\left(\lambda r_{1}\right) J_{1,2}\left(\lambda r_{2}\right) \lambda d \lambda I d_{N} \\
: L^{2}\left(\boldsymbol{R}^{+}, d r, V(k)\right) \rightarrow L^{2}\left(\boldsymbol{R}^{+}, d r, V(k)\right),
\end{aligned}
$$

etc., we can assert that, for the study of $k_{f}(; i)$, it suffices to study the linear operators

$$
\begin{aligned}
& \tilde{k}_{f}(; \tilde{c c}(i))=k_{f}(; \tilde{c c}(i)): L^{2}\left(\boldsymbol{R}^{+}, r^{m i-2 \imath} d r, L^{2} \Lambda^{i ; \tilde{c}}(N)\right) \\
& \rightarrow L^{2}\left(\boldsymbol{R}^{+}, r^{m-22} d r, L^{2} A^{i ; \tilde{c c}}(N)\right), \\
& \tilde{k}_{\tilde{f}}^{ \pm}(; \tilde{c e}(i)): L^{2}\left(\boldsymbol{R}^{\dagger}, r^{m-2 i} d r, L^{2} \Lambda^{2: \tilde{c \tilde{e}}}(N)\right) \\
& \rightarrow L^{2}\left(\boldsymbol{R}^{+}, r^{n-2 u} d r, L^{2} A^{i ; \tilde{c}}(N)\right), \\
& \left(r_{1} r_{2}\right)^{1 / 2} \int_{0}^{\infty} f\left(\lambda^{2}\right) J_{ \pm 1 / 2}\left(\lambda r_{1}\right) J_{ \pm 1 / 2}\left(\lambda r_{2}\right) \lambda d \lambda: L^{2}\left(\boldsymbol{R}^{+}, d r\right) \rightarrow L^{2}\left(\boldsymbol{R}^{+}, d r\right) .
\end{aligned}
$$

Finally, we will prove the Rellich-type theorem which is the fundamental material for constructing the Hodge theory in the next section.

The norm on dom $\bar{J}_{2}$ is defined by

$$
\|\theta\|_{1}=\|(I+\Delta) \theta\|_{C(x)} .
$$


THEOREM 3.8. Given $0<\varepsilon<\infty$, the restriction map

$$
\operatorname{dom} \bar{J}_{i} \rightarrow L^{2} A^{2}\left(C_{0, \varepsilon}(N)\right)
$$

is compact.

Proof. The proof is similar to that of [6] Theorem 3.4.

Given $0<r \leqq \varepsilon$, we have the inequality

$$
\left|J_{\nu}(r)\right| \leqq K \frac{r^{\nu}}{\Gamma(\nu+1)}, \quad \nu>-1,
$$

where $K=K(\varepsilon)>0$ is independent of $\nu$.

Take a form $\theta \in L^{2} \Lambda^{2}(C(N))$ with $\operatorname{supp} \theta \subset[0, \varepsilon] \times N$ and expand it to the convergent sum (2.6). Then, for $\nu>-1$, we have the following inequalities.

$$
\begin{gathered}
\left|H_{\nu}\left(r^{-\alpha(i)} g_{\tilde{c} \tilde{c}(i), j}\right)\right| \leqq K \frac{\lambda^{\nu}}{\Gamma(\nu+1)} \cdot \frac{a^{1+\nu}}{\sqrt{2+2 \nu}} \| g_{\tilde{c}(i), j}\left(\omega_{\tilde{c} \tilde{c}(i), j} \|_{C(\nu)},\right. \\
\left|H_{2}\left(r^{-\alpha(i-1)} f_{\tilde{c}(i-1), j}\right)\right| \leqq K \frac{\lambda^{\nu}}{\Gamma(\nu+1)} \cdot \frac{a^{1+\nu}}{\sqrt{2+2 \nu}}\left\|f_{\tilde{c}(i-1), j} d r \wedge \omega_{\tilde{c}(i-1), j}\right\|_{C(N)} .
\end{gathered}
$$

Moreover, let $\left\{\left(G_{j}(r), F_{j}(r)\right)\right\}$, be the image of the second sum of the r.h.s. of (2.6) with respect to the map (3.45). Then, for $\nu>0$, we have the following inequalities.

$$
\left|H_{\nu-1}\left(G_{j}\right)\right| \leqq K \frac{\lambda^{\nu-1}}{\Gamma(\nu)} \cdot \frac{a^{\nu}}{\sqrt{2 \nu}} \| g_{\tilde{c} \tilde{e}(\imath-1), j} \tilde{d} \omega_{c \tilde{e}(i-1), \jmath}
$$

$$
\begin{aligned}
& +f_{\tilde{c} \tilde{e}(i-1), j} d r \wedge \omega_{\tilde{c} \tilde{e}(i-1), j} \|_{C(N)}, \\
& \left|H_{\nu+1}\left(F_{j}\right)\right| \leqq K \frac{\lambda^{\nu+1}}{\Gamma(\nu+2)} \cdot \frac{a^{2+\nu}}{\sqrt{4+2 \nu}} \| g_{\tilde{c} \tilde{\varepsilon}(i-1), j} d \omega_{\tilde{c}(i-1), ~} \\
& +f_{\tilde{c \tilde{e}(i-1), j}} d r \wedge \omega_{\tilde{c} \tilde{e}(i-1), j} \|_{C(N)} .
\end{aligned}
$$

In Case A, consider the inequalities $(3.72)_{\nu(\tilde{c}(i)\rangle_{j}},(3.73)_{\nu(\tilde{c}(i-1), 2-2)}$, and $(3.74)_{\nu}\left(\tilde{c \tilde{e}(i-1))_{j}}\right.$. In Case $\mathrm{B}(V(k))$, consider $(3.72)_{\nu(V(k))_{j}},(3.72)_{-\nu(V(k)} \perp_{j},(3.72)_{\nu(\tilde{c} \tilde{e}(k))_{j}},(3.73)_{\nu(\tilde{c}(k-1), k-2)_{j}}$

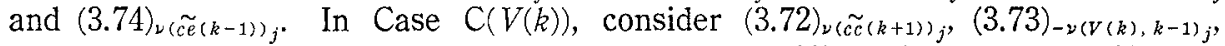
$(3.73)_{\nu\left(V(k)^{\perp}, k-1\right)_{j}},(3.73)_{\nu(\tilde{e}(k), k-1)}$, and $(3.74)_{\nu(\tilde{c} \tilde{e}(k))_{j}}$. Then, in any cases if $\mathscr{B}$ is contained in the unit ball in $L^{2} \Lambda^{2}(C(N))$ and consists of forms supported on $[0, \varepsilon] \times N$, then, for any fixed positive $a$, the map

$$
\mathscr{H}_{\nu}: L^{2} \Lambda^{2}(C(N)) \cong L^{2}\left(\boldsymbol{R}^{+}, \lambda d \lambda, l^{2} \oplus l^{2} \oplus l^{2} \oplus l^{2}\right)
$$

defined by (3.27), (3.33) and (3.49) (in Cases $\mathrm{B}(V(k))$ or $\mathrm{C}(V(k)), \Omega_{V(k) \perp,}^{+}$or $\Omega_{d r \wedge V(k), j}^{\dagger}$ should be replaced by $\Omega_{V(k) \perp, j}^{-}$or $\Omega_{d r \wedge V(k), j}$, respectively ; see Steps VI, VII) carries $\mathcal{B}$ into a family of forms whose restrictions to $(0, a)$ have range lying in a totally bounded subset of $l^{2} \oplus l^{2} \oplus l^{2} \oplus l^{2}$.

Hence the proof can be completed by the argument similar to that of [6] Theorem 3.4. 


\section{§4. Hodge theory for $X$ with $\bar{X}$ compact.} for $X$.

Let us return to our $X^{m+1}=C_{0, u}\left(N^{m}\right) \cup X_{u}$ and construct the Hodge theory

To avoid awkward repetitions of similar expressions, let's adopt the following notations and definitions.

First classify Case $A$ into

Case $A_{-1}: \operatorname{dim} N=2 k, \widetilde{\mathscr{A}}_{b c}^{k}(N) \neq\{0\}$ and $\imath=k-1$,

Case $A_{0}$ : otherwise.

Moreover, denote Case $A_{-1}$ with $V \subset \widetilde{\mathcal{H}}_{b c}^{k}(N)$ specified simply by Case $A_{-1}(V)$. Next we set

$$
\begin{gathered}
A^{2}(X)= \begin{cases}A_{b c}^{\imath}(X) ; & \text { in Case } \mathrm{A}, \\
A_{b c, V}^{\imath}(X) ; & \text { in Cases } \mathrm{B}(V), \mathrm{C}(V),\end{cases} \\
D_{\imath}=\left.d\right|_{\mathcal{A}(X),} D_{\imath}^{*}=\left.\delta\right|_{\mathcal{A}(t+1(X),}
\end{gathered}
$$

and denote the closures of $D_{\imath}, D_{\imath}^{*}$ by $\bar{D}_{\imath}, \bar{D}_{\imath}^{*}$ respectively. Then

$$
\begin{aligned}
& D_{\imath}= \begin{cases}d_{b c, \imath} ; & \text { in Case } \mathrm{A}, \\
d_{b c, V, \imath} ; & \text { in Case } \mathrm{B}(V),\end{cases} \\
& D_{\imath}^{*}= \begin{cases}\delta_{b c, \imath} ; & \text { in Cases } \mathrm{A}_{0} \text { or } \mathrm{C}(V), \\
\delta_{b c, V, \imath} ; & \text { in Case } \mathrm{B}(V),\end{cases}
\end{aligned}
$$

but the operators $D_{\imath}$ of Case $\mathrm{C}(V)$ and $D_{\imath}^{*}$ of Case $\mathrm{A}_{-1}(V)$ haven't appeared until now. However, we have

$$
\begin{aligned}
& \bar{D}_{\imath}=\bar{d}_{b c, \imath} ; \quad \text { in Case } \mathrm{C}(V), \\
& \bar{D}_{\imath}^{*}=\bar{\delta}_{b c, \imath} ; \quad \text { in Case } \mathrm{A}_{-1}(V) .
\end{aligned}
$$

In fact, (4.4) is an immediate consequence of the facts that the ideal boundary condition on $\theta \in \Lambda_{b c, V}^{k+1}(X)$ is concerned only with $\theta_{d r \wedge V}+\theta_{d r \wedge V^{\perp}}$ and moreover $d\left(\theta_{d r \wedge V}+\theta_{d r \wedge V^{\perp}}\right)=0$. (4.5) is also similarly derived. Therefore, in any cases, $\bar{D}_{\imath}^{*}$ is the Hilbert space adjoint of $\bar{D}_{\imath}$, and our Laplacian $\bar{J}_{\imath}$ can be written as follows :

$$
\bar{J}_{\imath}=\bar{D}_{\imath}^{*} \bar{D}_{i}+\bar{D}_{\imath-1} \bar{D}_{\imath-1}^{*}: L^{2} \Lambda^{\imath}(X) \rightarrow L^{2} \Lambda^{2}(X)
$$

Further, if we set

$$
\begin{gathered}
\mathscr{M}^{2}(X)=\left\{\theta \in L^{2} A^{2}(X) \mid d \theta=\delta \theta=0 \text { as distributions. }\right\}, \\
\begin{cases}\mathscr{H}_{b c}^{c}(X)=\mathscr{H}^{2}(X) \cap \operatorname{dom} \bar{d}_{b c, i} \cap \operatorname{dom} \bar{\delta}_{b c, \ell-1} & ; \text { in Case } A, \\
\mathscr{H}_{b c, V}^{k}(X)=\mathscr{H}^{k}(X) \cap \operatorname{dom} \bar{d}_{b c, V, k} \cap \operatorname{dom} \bar{\delta}_{b c, k-1} & ; \text { in Case } B(V), \\
\mathscr{H}_{b c, V}^{k+1}(X)=\mathscr{H}^{k+1}(X) \cap \operatorname{dom} \bar{d}_{b c, k+1} \cap \operatorname{dom} \bar{\delta}_{b c, V, k} ; \text { in Case } C(V),\end{cases}
\end{gathered}
$$


then $(4.8)$ equals to

$$
\mathscr{H}^{2}(X)=\mathscr{H}^{\prime}(X) \wedge \operatorname{dom} \bar{D}_{i} \cap \operatorname{dom} \bar{D}_{i-1}^{*}
$$

in respective cases.

From now on, we will use mainly the notations $\mathcal{A}^{i}(X), \bar{D}_{\iota}, \bar{D}_{\imath}^{*}, \mathscr{H}^{i}(X)$ and return to use $A_{b c}(X), \bar{d}_{b c, v}$, etc. when occasion demands.

General properties of $\bar{J}_{2}$ and objects around it can be derived by the method similar to [7]. Hence we omit explaining such general ones and we concentrate ourselves on constructing the Hodge theory when $\bar{X}$ is compact.

In the following, the metric completion $\bar{X}$ of our $X$ is assumed to be compact.

LEAINA 4.1. Given $\lambda>0$, the operator

$$
\left(\bar{J}_{i} \perp \lambda\right)^{-1} \cdot L^{2} A^{2}(X) \rightarrow L^{2} A^{2}(X)
$$

is compact.

Proof. $\left(\bar{J}_{i} \div \lambda\right)^{-1}: L^{2} A^{2}(X)$ dom $J_{2}$ is a continuous operator. Hence, by Theorem 3.8 and the well-known Rellich theorem for $X_{u},(4.10)$ is compact.

Thus, given $\lambda>0,(4.10)$ is a compact self-adjoint operator. Therefore, by the general theory of Riesz-Schauder, we get

Proposition 4.2. The spectrum of $\bar{J}_{2}$ consısts of eigenvalues of finite multzplicity.

Moreover we have

$$
\mathscr{H}^{2}(X)=\left\{\theta=\operatorname{dom} \bar{J}_{\imath} \mid \bar{J}_{i} \theta=0\right\},
$$

and, by the general theory of Fredholm, for a given $f \in L^{2} \Lambda^{2}(X)$, there exists $\theta \in \operatorname{dom} \bar{J}_{2}$ satisfying $\bar{J}_{i} \theta=f$ if and only if $f$ is orthogonal to $\mathscr{H}^{i}(X)$. Hence, if we define $H_{\imath}: L^{2} A^{2}(X) \rightarrow \mathscr{Y}^{i}(X)$ to be the orthogonal projection, then, for a given $\theta \in L^{2} \Lambda^{2}(X)$, there exists a unique element $\theta_{1} \in \operatorname{dom} \bar{D}_{2}$ which satisfies $\bar{\Delta}_{i} \theta_{1}=$ $\theta-H_{2} \theta$ and $H_{i} \theta_{1}=0$, by which the Green's operator $G_{i}$ is defined to be $G_{i} \theta=\theta_{1}$.

THEOREM 4.3 (Hodge decomposition). There exists the unique pair of continuous operators

$$
H_{1}, G_{1} \cdot L^{2} A^{2}(X) \rightarrow \operatorname{dom} J_{3}
$$

such that $J_{1} H_{b}=H_{i} G_{2}=0$ and, for any $\theta \in L^{2} A^{2}(X)$,

$$
\theta=H_{i} \theta \div \bar{D}_{\imath}^{*} \bar{D}_{i} G_{i} \theta \div \bar{D}_{\imath-1} \bar{D}_{\imath-1}^{*} G_{\imath} \theta
$$

The uniqueness can be easily verified.

Next we will study the harmonic space $\mathscr{H}^{i}(X)$. Then $z$-th $L^{2}$-cohomology group of $X$ is defined by 


$$
H_{(2)}^{i}(X)=\operatorname{Ker} \bar{D}_{\imath} / \text { Range } \bar{D}_{\iota-1} .
$$

If necessary to indicate the boundary condition or the ideal one, we will use the notations $H_{(2), b c}^{i}(X), H_{(2), b c, t}^{i}(X)$ instead of (4.14).

LEMMA 4.4. The natural map $\omega \mapsto[\omega]$ induces the isomorphs:

$$
\iota_{\mathscr{H}}: \mathscr{H}^{2}(X) \cong H_{(, 2)}^{i}(X) \text {. }
$$

Proof. We have the Hodge decomposition

$$
L^{2} A^{i}(X)=H^{i}(X) \subseteq \text { Range } \bar{D}_{t}^{*}=\text { Range } \bar{D}_{1-1} .
$$

And we have

$$
\operatorname{Ker} \bar{D}_{\imath} \cdots \mathscr{H}^{2}(X) \cup \text { Range } \bar{D}_{t-1} \text {. }
$$

Thus the proof is complete.

THeOREM 4.5. Set $\hat{\partial X}=\hat{\partial X} \frown \hat{\partial} X_{u}, \quad Y_{u} \cdots \overline{C_{u, u}(\backslash)} \frown \partial X_{u}$.

$$
\begin{aligned}
& H_{(2), a b s}^{i}(X) \cong \begin{cases}H^{l}\left(X_{u}\right) & ; \quad l<\left[\begin{array}{c}
m-1 \\
2
\end{array}\right], \\
H^{\prime}\left(X_{u}, X_{u}\right) & ; \quad i>\left[\begin{array}{c}
m \div 1 \\
2
\end{array}\right] \div 1,\end{cases} \\
& H_{(u), \operatorname{rel}}^{2}(X) \cong \begin{cases}H^{i}\left(X_{u}, \hat{\partial X_{u}}\right), & \iota<\left[\begin{array}{c}
m+1 \\
2
\end{array}\right], \\
H^{i}\left(X_{n}, \partial X_{u}\right) & ; \quad i>\left[\begin{array}{c}
m+1 \\
2
\end{array}\right]-1 .\end{cases}
\end{aligned}
$$

(2) In Case where $m=2 k+1$,

$$
\begin{aligned}
& H_{(2), a b s}^{i}(X) \cong \begin{cases}i^{*}\left(H^{k}\left(X_{u}, V_{u}\right)\right) \subset H^{k}\left(X_{u}\right) ; & i=k=\left[\begin{array}{c}
m-1 \\
2
\end{array}\right] \\
H^{k+1}\left(X_{u}, V_{u}\right) & ; \quad l=k-\div, 1,\end{cases} \\
& H_{(2), r u t}^{i}(X) \cong \begin{cases}i^{*}\left(H^{k}\left(X_{u}, X_{u}\right)\right) \subset H^{k}\left(X_{u}, \hat{o X_{u}}\right) ; & l=k, \\
H^{k+1}\left(X_{u}, \hat{\partial} X_{u}\right) & ; \quad l=k-1 .\end{cases}
\end{aligned}
$$

(3) In Case where $m=2 k$, if $\tilde{\mathscr{H}}_{b c}^{b}(N)=\{0\}$, then

$$
H_{(\xi), b c}^{i}(X)=H_{(2), b r, 10\}}^{i}(X) ; \quad l \cdots k, k \cdots 1,
$$

and, in general, given $V \subset \widetilde{\mathcal{H}}_{b c}^{k}(N)$, we have

$$
H_{(2), a b s, V}^{2}(X) \cong \begin{cases}\left(j^{*}\right)^{-1}\left(V^{\perp}\right) \subset H^{k}\left(X_{u}\right) & ; \quad \imath=k=\left[\begin{array}{c}
m \div 1 \\
2
\end{array}\right], \\
H^{k+1}\left(X_{u}, N_{u}\right) / \delta^{*}\left(V^{\perp}\right) ; & \imath=k+1 .\end{cases}
$$




$$
H_{(2), r \ell l, V}^{i}(X) \cong \begin{cases}\left(j^{*}\right)^{-1}\left(V^{\perp}\right) \subset H^{k}\left(X_{u}, \hat{\partial X_{u}}\right) ; i=k, \\ H^{k+1}\left(X_{u}, \partial X_{u}\right) / \delta^{*}\left(V^{\perp}\right) ; i=k+1 .\end{cases}
$$

In each case, the isomorphism is given as follows :

Given $[\omega]$, that is, an element of the right hand side of each expression, let's extend $\left.\omega\right|_{N_{u}}$ to $\tilde{\omega}$, a form on $C_{0, u}(N)$, and denote the form thus defined on $X$ by $\Omega$, that is, $\Omega=\tilde{\omega}$ on $C_{0, u}(N)$ and $\Omega=\omega$ on $X_{u}$. Then $[\Omega]$ is defined to be the image of $[\omega]$ with respect to our isomorphism.

Besides, each right hand side of $(4.20)$ and (4.23) is defined by the long exact sequence

$$
\cdots \longrightarrow H^{k}\left(X_{u}, N_{u}\right) \stackrel{i^{*}}{\longrightarrow} H^{k}\left(X_{u}\right) \stackrel{j^{*}}{\rightarrow} H^{k}\left(N_{u}\right) \stackrel{\delta^{*}}{\longrightarrow} H^{k+1}\left(X_{u}, N_{u}\right) \longrightarrow \cdots
$$

and the orthogonal decomposition $H^{k}\left(N_{u}\right)=V \oplus V^{\perp}$. Moreover, each right hand side of (4.21) and (4.24) is defined by the long exact sequence

$$
\begin{aligned}
\cdots \longrightarrow H^{k}\left(X_{u}, \partial X_{u}\right) \stackrel{i^{*}}{\longrightarrow} H^{k}\left(X_{u}, \hat{\partial X_{u}}\right) \stackrel{j^{*}}{\longrightarrow} H^{k}\left(N_{u}, \partial N_{u}\right) & \\
& \stackrel{\delta^{*}}{\longrightarrow} H^{k+1}\left(X_{u}, \partial X_{u}\right) \longrightarrow \cdots
\end{aligned}
$$

and the orthogonal decomposition $H^{k}\left(N_{u}, \partial N_{u}\right)=V \oplus V^{\perp}$.

Proof of Theorem 4.5. Referring to [4], [16], etc., let's gather some properties of the $L^{2}$-cohomology groups. Set $C=C_{0, u}(N)$. Let's define the $t$-th relative $L^{2}$-cohomology group $H_{(2)}^{2}(X, C)$ to be the $\imath$-th cohomology group of the cochain complex

$$
\left(\left\{\theta \in \operatorname{dom} \bar{D}_{*} \mid \theta_{1 C}=0\right\}, \bar{D}_{*,(X, C)}=\bar{D}_{*}\right) .
$$

Then, we have the $L^{2}$-version of long exact sequence

$$
\begin{aligned}
\cdots \longrightarrow H_{(2)}^{i-1}(C) \longrightarrow H_{(2)}^{i}(X, C) \longrightarrow H_{(2)}^{i}(X) & \longrightarrow H_{(2)}^{i}(C) \\
& \longrightarrow H_{(2)}^{i+1}(X, C) \longrightarrow \cdots
\end{aligned}
$$

We remark that, when we define $H_{(2)}^{*}(C)$, we leave the boundary condition on $N_{u}$ out of consideration.

Moreover, we have

$$
\begin{aligned}
& H_{(2), a b s}^{i}(X, C) \cong H^{i}\left(X_{u}, N_{u}\right) \\
& H_{(2), r e l}^{i}(X, C) \cong H^{i}\left(X_{u}, \partial X_{u}\right) \\
& H_{(2), a b s, V}^{i}(X, C) \cong H^{i}\left(X_{u}, N_{u}\right) \quad \text { in Case } A, \\
& H_{(2), r e t, V}^{i}(X, C) \cong H^{i}\left(X_{u}, \partial X_{u}\right) \quad \text { in Cases } B(V), C(V) .
\end{aligned}
$$

Since the ideal boundary condition has no effect on our relative $L^{2}$-cohomology groups, in view of $(0.9)$ and (1.3), it suffices to prove 
Ker $\bar{d}_{\imath,(X, C)} /$ Range $\tilde{d}_{\imath-1,(X, C)} \cong H^{i}\left(X_{u}, N_{u}\right)$,

$$
\text { Ker } \bar{d}_{c, \imath,(X, C)} / \text { Range } \bar{d}_{c, \imath-1,(X, C)} \cong H^{i}\left(X_{u}, \partial X_{u}\right) \text {. }
$$

(4.30) can be shown similarly to [16] Lemma 3.14. As for (4.31), if we take a manifold $\hat{X}_{u}$ with $\hat{X}_{u} \supset$ Int $\hat{X}_{u} \supset X_{u}$, then the left hand side of (4.31) is naturally isomorphic to

$$
\operatorname{Ker} \bar{d}_{\imath,\left(\hat{x}_{u}, \hat{x}_{u}-x_{u}\right)} / \text { Range } \bar{d}_{\imath-1,\left(\hat{x}_{u}, \hat{x}_{u}-x_{u}\right)} \text {. }
$$

And this is naturally isomorphic to the right hand side of (4.31); see [16] Lemma 3.14. Thus (4.28) and (4.29) have been proved.

Next, consider $H_{(2)}^{*}(C)$. Precisely writing, $H_{(2)}^{i}(C)=H_{(2), b e}^{i}(C)$ in Case $A$ and $H_{(2)}^{i}(C)=H_{(2), b c, V}^{i}(C)$ in Cases $B(V), C(V)$. We have

$$
H_{(2), b c}^{i}(C) \cong \begin{cases}H_{(2), b c}^{i}(C) ; & i<\frac{m+1}{2} \\ \{0\} & ; \quad i \geqq \frac{m+1}{2} .\end{cases}
$$

We remark that we are taking account of Cases $B(\{0\}), C(\{0\})$, too, by the identification

$$
H_{(2), b c, 10\}}^{i}(C)=H_{(2), b c}^{i}(C) \quad \text { in Cases } B(\{0\}), C(\{0\}) .
$$

(4.32) can be shown similarly to [4] Lemma 3.4. Moreover we have

$$
\begin{aligned}
& H_{(2), a b s}^{i}\left(N_{u}\right) \cong H^{i}\left(N_{u}\right), \\
& H_{(2), r e l}^{i}\left(N_{u}\right) \cong H^{2}\left(N_{u}, \partial N_{u}\right) .
\end{aligned}
$$

These can be shown by the arguments similar to the proofs of (4.30) and (4.31). Moreover, in Case $B(\{0\})$, by (4.32)-(4.34), we have gotten the isomorphism

$$
\begin{array}{cc}
\widetilde{\mathscr{T}}_{b c}^{k}(N) \cong H_{(2), b c, 0\}}^{k}(C), \\
\Psi & \Psi \\
\omega & \longmapsto
\end{array}
$$

where $\tilde{\omega}(r, \tilde{x})=\omega(\tilde{x})$. Now, in Case $B(V)$, through (4.35), we get the isomorphism

$$
\begin{gathered}
V^{\perp} \cong H_{(2), b c, V}^{k}(C)=\operatorname{Ker} \bar{d}_{b c, V, k} / \text { Range } \bar{d}_{b c, k-1} \\
\cap{ }_{\mathscr{T}_{b c}^{k}}(N) \cong H_{(2), b c, 0\}}^{k}(C)=\operatorname{Ker} \bar{d}_{b c, k} / \text { Range } \bar{d}_{b c, k-1} .
\end{gathered}
$$

Besides, in Case $C(\{0\})$, by (4.32) and (4.33), we know

$$
\text { Ker } \bar{d}_{b c, k+1} / \text { Range } \bar{d}_{b c, 101, k} \cong\{0\} \text {. }
$$

In Case $C(V)$, we can show

$$
\text { Ker } \bar{d}_{b c, k+1} / \text { Range } \bar{d}_{b c, V, k} \cong\{0\} \text {, that is, }
$$




$$
H_{(2), b c, V}^{k+1}(C) \cong\{0\} .
$$

In fact, if we decompose $\alpha \in \operatorname{Ker} \bar{d}_{b c, k+1}$ into

$$
\alpha=\phi+d r \wedge \omega,
$$

then $\alpha=d \int_{0}^{r} \omega$ with $\int_{0}^{r} \omega \subseteq \operatorname{dom} \bar{d}_{b c, k}$; see (3.12) and Lemma 3.2 of [4]. Moreover, obviously, $\int_{0}^{r} \omega \in \operatorname{dom} \vec{d}_{b c, \mathscr{H}_{b c}^{k}(w), k}$. Thus (4.38) was proved.

We can now prove the theorem by applying the five lemma repeatedly to the diagrams which are made by arranging (4.25), (4.26) and (4.27) suitably. Here we will give only the diagram corresponding to " $b c$ "- "abs".

The subscripts " $a b s$ " and " $V$ " are removed in the following.

In the case where $\imath<\left[\begin{array}{c}m+1 \\ 2\end{array}\right]$, by $(4.28),(4.32)$ and $(4.34)$, we have the following commutative diagram.

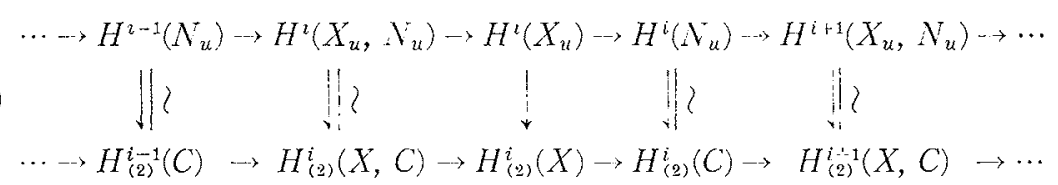

In the case where $i>\left[\begin{array}{c}m+1 \\ 2\end{array}\right]-1$, or in the case where $m=2 k-1$ and $\imath=k+1$, by (4.28), (4.32) and (4.38), we have the following commutative diagram.

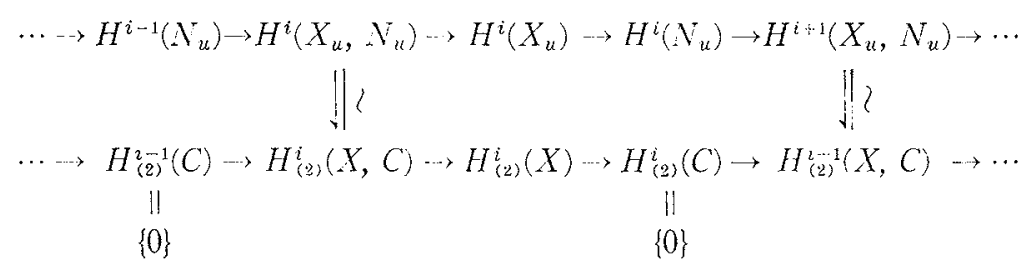

In the case where $m=2 k-1$, by $(4.28),(4.32)$ and (4.34), we have the following commutative diagram.

$$
\cdots \rightarrow H^{k-1}\left(N_{u}\right) \rightarrow H^{k}\left(X_{u}, N_{u}\right) \rightarrow H^{k}\left(X_{u}\right) \rightarrow H^{k}\left(N_{u}\right) \rightarrow H^{k+1}\left(X_{u}, N_{u}\right) \rightarrow \cdots
$$

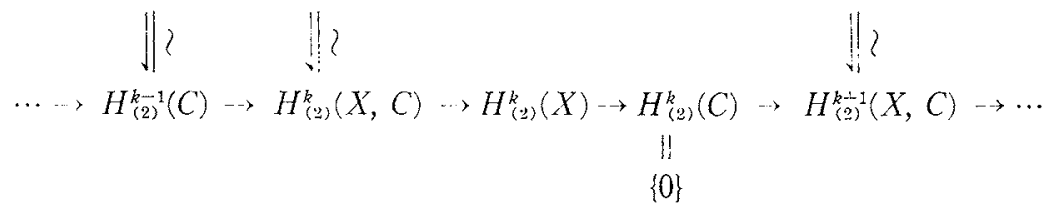

Finally, in the case where $m-2 k$, by (4.29), (4.36) and (4.38), we have the following commutative diagram. 


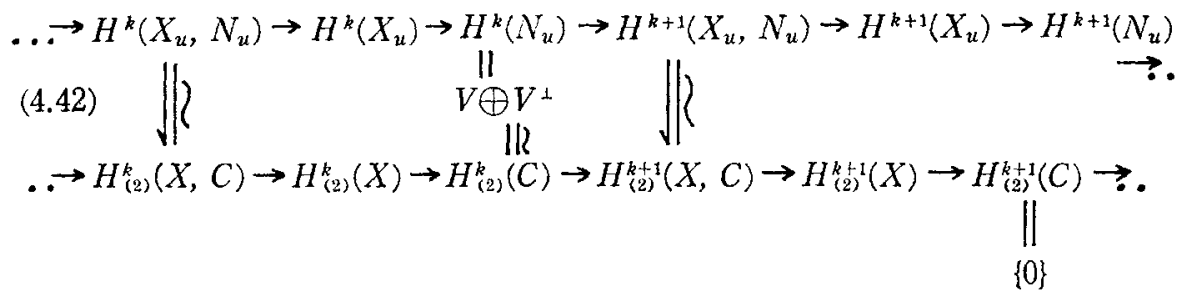

Finishing our study of the harmonic space, we next aim to study the heat kernel and the objects around it in the following sections.

In the following, our $X$ is always assumed to have the compact metric completion $\bar{X}$. Moreover, let's denote the sequence of eigenvalues of $\bar{D}_{2}$ by

$$
\left(0 \leqq \lambda_{\imath, 0} \leqq \lambda_{\imath, 1} \leqq \lambda_{\imath, 2} \leqq \cdots \uparrow \infty\right.
$$

and denote the orthonormal basis of $L^{2} A^{2}(X)$ consisting of eigenforms corresponding to (4.43) by

$$
\varphi_{i, 0}, \varphi_{i, 1}, \varphi_{i, 2}, \cdots
$$

If necessary, the subscripts " $b c$ ", "V" are added, for examples, $\left\{\vec{J}_{b c, \iota}, \lambda_{b c, \downarrow, \nu}\right.$, $\left.\varphi_{b c, \imath, j}\right\}$ in Case $A$, or $\left\{\bar{d}_{b c, V, \imath}, \lambda_{b c, V, \imath, 2}, \varphi_{b c, V, \imath, j}\right\}$ in Cases $B(V), C(V)$.

\section{§. Heat kernel.}

The main purpose of this section is to investigate the asymptotic behavior of the trace of the heat kernel on $X^{m+1}=C_{0, u}(N) \cup X_{u}$;

$$
e^{-t \bar{d}_{i}}=\sum_{j} e^{-t \lambda_{\imath, j}} \varphi_{i, j}\left(x_{1}\right) \otimes \varphi_{i, j}\left(x_{2}\right) .
$$

To do so, it is best to reconstruct the heat kernel on $X$ according to the method of E.E. Levi from the one on $C(N)$ and the one on a complement of a neighborhood of the singular point; the last two heat kernels are accessible to us.

We need the detailed description of the reconstruction as follows.

Let $E_{C}\left(t, x_{1}, x_{2} ; i\right)$ be the heat kernel for $i$-forms on $C(N)$, which has the formal expression $k_{e}-\lambda t\left(x_{1}, x_{2} ; i\right)$ according to $\S 3$; here we impose on $E_{C}(; i)$ the boundary condition and (in certain cases) the ideal boundary condition which are consistent with those of (5.1). Next, let $\varepsilon^{\prime}>0$ be sufficiently small and $\left(X_{\varepsilon^{\prime}}^{*}, N_{\varepsilon^{\prime}}^{*}\right)$ be the copy of $\left(X_{\varepsilon^{\prime}}, N_{\varepsilon^{\prime}}\right)$, where $N_{\varepsilon^{\prime}}=\overline{C_{0, \varepsilon^{\prime}}(N)} \cap X_{s^{\prime}}$. Then $M$ is defined to be the attaching space $X_{\varepsilon^{\prime}} \cup_{I} X_{\varepsilon^{\prime}}^{*}$, where $I$ is the natural identification map $N_{c^{\prime}} \cong N_{\varepsilon^{\prime}}^{*}$; $M$ is endowed with the Riemannian metric which coincides with the given metric on $X_{\varepsilon^{\prime}}$. Let $E_{M}\left(t, x_{1}, x_{2} ; i\right)$ be the heat kernel for $i$-forms on $M$ which is thus a compact Riemannian manifold possibly with smooth boundary ; here we impose on $E_{M}(; i)$ the boundary condition which is consistent with that of $(5.1)$.

Fix $\varepsilon>0$ such that $0<\varepsilon^{\prime}<\varepsilon<6 \varepsilon<u$. 
Let $\left\{\Phi_{C}(x), \Phi_{M}(x)\right\}$ be a partition of unity on $X$ such that both elements depend only on $r$ when $x=(r, \tilde{x}) \in C_{0, u}(N)$, supp $\Phi_{C} \subset C_{0,4 \varepsilon}(N)$, supp $\Phi_{M} \subset X_{3 \varepsilon}, \Phi_{C}=1$ on $C_{0,3 \varepsilon}(N)$ and $\Phi_{M}=1$ on $X_{4 \varepsilon}$. Moreover, let $\Psi_{C}(x), \Psi_{M}(x)$ be smooth functions on $X$ such that both functions depend only on $r$ when $x=(r, \tilde{x}) \in C_{0, u}(N)$, supp $\Psi_{C}$ $\subset C_{0,6 \varepsilon}(N)$, supp $\Psi_{M} \subset X_{\varepsilon}, \Psi_{C}=1$ on $C_{0,5 \varepsilon}(N)$ and $\Psi_{M}=1$ on $X_{2 \varepsilon}$.

Now set

$$
\begin{aligned}
\tilde{E}\left(t, x_{1}, x_{2} ; i\right)= & \Psi_{C}\left(x_{1}\right) E_{C}\left(t, x_{1}, x_{2} ; i\right) \Phi_{C}\left(x_{2}\right) \\
& +\Psi_{M}\left(x_{1}\right) E_{M}\left(t, x_{1}, x_{2} ; i\right) \Phi_{M}\left(x_{2}\right) .
\end{aligned}
$$

As usual we look for a double form $F\left(t, x_{1}, x_{2} ; i\right)$ such that the double form

$$
\begin{aligned}
E\left(t, x_{1}, x_{2} ; i\right)= & \tilde{E}\left(t, x_{1}, x_{2} ; i\right) \\
& -\int_{0}^{t} d t_{1} \int_{X} \tilde{E}\left(t-t_{1}, x_{1}, x_{3} ; i\right) \wedge *_{x_{3}} F\left(t_{1}, x_{3}, x_{2} ; i\right)
\end{aligned}
$$

satisfies

$$
\begin{gathered}
E\left(+0, x_{1}, x_{2} ; i\right)=I, \\
\left(\frac{\partial}{\partial t}+\Delta_{x_{1}}\right) E\left(t, x_{1}, x_{2} ; i\right)=0 .
\end{gathered}
$$

We will search for $F\left(t, x_{1}, x_{2} ; i\right)$ with the following two properties (I), (II).

( I ) $F\left(t, x_{1}, x_{2} ; i\right)$ is a smooth double- $i$-form on $[0, \infty) \times X \times X$ which has support on $[0, \infty) \times\left(C_{\varepsilon, 2 \varepsilon}(N) \cup C_{5 \varepsilon, 6 \varepsilon}(N)\right) \times X$ and, for fixed $t$ and $x_{2}$, belongs to $\operatorname{dom} \bar{A}_{\imath}$ with respect to $x_{1}$.

(II) Let $\varepsilon^{\prime \prime}<1$ be sufficiently near 1 and $\varphi(x)$ be a smooth function on $X$ such that $\varphi(x)>0, \varphi(r, \tilde{x})=r^{-\left(m+\varepsilon^{n}\right\rangle / 2}$ on $C_{0, \varepsilon / 2}(N)$ and $\varphi(x)=1$ on $X_{\varepsilon}$; see (5.43). Then there exist $K>0$ and $\delta>0$ such that, for $n=0$ or 1 ,

$$
\left|\Delta^{n} x_{1} F\left(t, x_{1}, x_{2} ; i\right)\right| \leqq K \varphi\left(x_{2}\right) e^{-\delta / t} .
$$

Here, $|\cdot|$ is the pointwise norm at $\left(x_{1}, x_{2}\right)$ defined by $(0.3)$.

First of all, we will show that, if $F$ has the properties (I), (II), then $E\left(t, x_{1}, x_{2} ; i\right)$ satisfies (5.4) and, for any $\theta \in L^{2} \Lambda^{2}(X)$ and $t>0, E(t) \theta$ belongs to $\operatorname{dom} \bar{J}_{1}$.

Since, from (5.6), we have

$$
\begin{aligned}
\left|\int_{X} F\left(t_{1}, x_{3}, x_{2} ; i\right) \wedge *_{x_{2}} \theta\left(x_{2}\right)\right| & \leqq K_{1} \int_{X}\left|F\left(t_{1}, x_{i}, x_{2} ; i\right)\right|^{2} *_{x_{2}} 1\|\theta\|_{X}, \\
\int_{C_{0, \varepsilon / 2}(N)}\left|F\left(t_{1}, x_{3}, x_{2} ; i\right)\right|^{2} *_{x_{2}} 1 & \leqq K \cdot \operatorname{vol}(N) e^{-\delta / t} \int_{0}^{\xi / 2} r^{-\varepsilon^{\prime \prime}} d r \\
& =K \cdot \operatorname{vol}(N) \frac{(\varepsilon / 2)^{1-\varepsilon^{*}}}{1-\varepsilon^{\prime \prime}} e^{-\delta / t}
\end{aligned}
$$


the form $\int_{X} F\left(t_{1}, x_{3}, x_{2} ; i\right) \wedge *_{x_{2}} \theta\left(x_{2}\right)$ exists. Moreover, by estimating $\Delta_{x_{3}} \int_{X} F\left(t_{1}, x_{3}, x_{2} ; i\right) \wedge *_{x_{2}} \theta\left(x_{2}\right)$ similarly, we know that the above form belongs to $C^{0}\left([0, \infty)\right.$, dom $\left.\vec{J}_{\imath}\right)$. On the other hand, $E_{C}$ and $E_{M}$ define

$$
\begin{aligned}
& E_{C}: \operatorname{dom} \bar{J}_{i} \rightarrow C^{0}\left([0, \infty), \operatorname{dom} \bar{J}_{\imath}\right) \text { on } C(N), \\
& E_{M}: \operatorname{dom} \bar{J}_{i \rightarrow C^{0}}\left([0, \infty), \operatorname{dom} \bar{J}_{\imath}\right) \text { on } M \text {. }
\end{aligned}
$$

We examine only (5.7). By using the operator (3.75), we have

$$
\left(E_{C} \theta\right)(t,(r, \tilde{x}))=\mathscr{H}_{\nu}^{-1}\left(e^{-t \lambda^{2}} \mathscr{H}_{\nu}(\theta)\right)(r, \tilde{x}) .
$$

Hence, if $\theta \in \operatorname{dom} \bar{J}_{2}$ on $C(N)$, we have, for arbitrarily fixed $a>0$,

$$
\begin{aligned}
& \left\|(I+\Delta)\left(E_{C}(t) \theta-E_{C}(s) \theta\right)\right\|_{C(N)} \\
& \leqq K_{1}\left\|\left(1+\lambda^{2}\right)\left(e^{-t \lambda^{2}}-e^{-8 \lambda^{2}}\right) \mathscr{H}_{\nu}(\theta)\right\|_{L^{2}\left(R^{+}, \lambda d \lambda, l 2 \oplus l 2 \oplus l 2 \oplus l 2\right)} \\
& \leqq|t-s| K_{2}\left\|\left(1+\lambda^{2}\right) \mathscr{H}_{\nu}(\theta)\right\|_{L^{2}\left((0, a], \lambda d \lambda, 22 \oplus l 2 \oplus l 2 \oplus l^{2}\right)} \\
& +2 K_{1}\left\|\left(1+\lambda^{2}\right) \mathscr{H}_{\nu}(\theta)\right\|_{L^{2}\left([\alpha, \infty), \lambda d \lambda, l 2 \oplus l^{2} \oplus l 2 \oplus l 2\right)} .
\end{aligned}
$$

This implies (5.7). Since (5.7) and (5.8) induce

$$
\tilde{E}: \operatorname{dom} \bar{J}_{i \rightarrow} \rightarrow C^{0}\left([0, \infty) \text {, dom } \bar{J}_{3}\right) \quad \text { on } X
$$

we know that, for any $\theta \in L^{2} \Lambda^{2}(X), \int_{X} \tilde{E}\left(t, x_{1}, x_{3} ; i\right) \wedge *_{x_{3}} \int_{X} F\left(t_{1}, x_{3}, x_{2} ; i\right) \wedge *_{x_{2}} \theta\left(x_{2}\right)$ is a dom $\bar{\Delta}_{i}$-valued continuous function of $\left(t, t_{1}\right) \in[0, \infty) \times[0, \infty)$, and, moreover, $\int_{0}^{t} d t_{1} \int_{X} \tilde{E}\left(t-t_{1}, x_{1}, x_{3} ; i\right) \wedge *_{x_{3}} \int_{X} F\left(t_{1}, x_{3}, x_{2} ; i\right) \wedge *_{x_{2}} \theta\left(x_{2}\right)$ belongs to dom $\bar{J}_{2}$ and converges to 0 when $t \downarrow 0$. Moreover, by the argument similar to the proof of (5.10), we have

$$
\begin{gathered}
\tilde{E}: L^{2} A^{2}(X) \rightarrow C^{0}\left(\boldsymbol{R}^{+}, \operatorname{dom} J_{2}\right) \cap C^{0}\left([0, \infty), L^{2} A^{2}(X)\right), \\
\tilde{E}\left(+0, x_{1}, x_{2} ; i\right)=I .
\end{gathered}
$$

Then the desired results are immediate consequences of (5.11), (5.12) and the facts mentioned above.

Now, for constructing such a form $F$, we need some preparations. $E_{M}$ is well known ( $[17] \S 5)$, but all we know about $E_{C}$ now is that it can be written as a formal sum according to $\S 3$. Hence we need to investigate $E_{C}$ more closely.

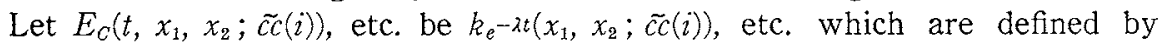
(3.3)-(3.7). Moreover, define $Z\left(t, x_{1}, x_{2} ; \tilde{c c}(i)\right)$, etc. by the following formulas.

$$
E_{c}(; \tilde{c c}(i))=\left(r_{1} r_{2}\right)^{\alpha(i-1)} e^{-\left(r_{1}-r_{2}\right)^{2} / 4 t} Z(; \tilde{c c}(i))
$$




$$
\begin{aligned}
& \left.E_{i:} ; d \overrightarrow{c e}(i)+d r \wedge \widetilde{c e}(i)\right) \\
& =\left(r_{1} r_{2}\right)^{\alpha(i)} e^{-\left(r_{1}-r_{2}\right)^{2} / t t} Z(; d \tilde{c e}(i)+d r \wedge \tilde{c e}(i)) \\
& =\left(r_{1} r_{2}\right)^{n(i)} e^{-\left(r_{1}-r_{2}\right)^{2 / 4 t}}\{Z(; d \tilde{c} \tilde{e}(i), d \tilde{c} \tilde{e}(i)) \\
& --Z(; d \tilde{e}(i), d r \wedge \tilde{c}(i))+Z(; d r \wedge \tilde{c}(i), d \tilde{e}(i)) \\
& -Z(; d r \wedge \tilde{c e}(i), d r \wedge \tilde{c e}(i))\} \\
& \left.E_{c i}, d r \wedge \tilde{c}(i)\right)=\left(r_{1} r_{2}\right)^{a(i)} e^{-\left(r_{1}-r_{2}\right)^{2} / t t} Z(; d r \wedge \tilde{c}(i)) \\
& \left.E \ddot{\bar{c}}^{(i} ; T(i)\right)=\left(r_{1} r_{2}\right)^{\alpha(i-1)} e^{-\left(r_{1}-r_{2}\right)^{2} / 4 t} Z=(; V(i)) \\
& \left.E_{i^{i}}^{-i}, d r \wedge V(i)\right)=\left(r_{1} r_{2}\right)^{\alpha(i)} e^{-\left(r_{1}-r_{2}\right)^{2} / t t} Z=\left(; d r \wedge V^{\prime}(i)\right) \\
& E_{c}(, i) \cdots\left(r_{1} r_{2}\right)^{a(i-1)} e^{-\left(r_{1}-r_{2}\right)^{2 / 1 t}} Z(; i) \text {. }
\end{aligned}
$$

Besides, $Z(; \pi)$, $(i)$, etc. are defined in the same way.

Set $\tau=t /\left(r_{1} r_{2}\right)$. Then, by Weber's second exponential integral ([14] III Page 200), we get, for $2>-1$.

$$
\begin{aligned}
\int_{0} e^{-t \lambda^{2}} J_{2}\left(\lambda r_{1}\right) J_{2}\left(\lambda r_{2}\right) \lambda d \lambda & =\frac{1}{2 t} e^{-\left(r_{1}^{9}+r_{2}^{2}\right) / 4 t} I_{\nu}\left(\frac{r_{1} r_{2}}{2 t}\right) \\
& =\left(r_{1} r_{2}\right)^{-1} e^{-\left(r_{1}-r_{2}\right) 2 / 4 t} \frac{1}{2 \tau} e^{-1 / 2=} I_{2}\left(\frac{1}{2 \tau}\right) .
\end{aligned}
$$

Here, $I_{\nu}$ is the modified Bessel function of order $\nu$.

Hence we have

$$
Z\left(; \tilde{c}(i)=\frac{1}{2 \tau} e^{-1 ; \nu} \sum_{j} I_{\nu(\tilde{c}(i)),}\left(\frac{1}{2 \tau}\right) \omega_{\tilde{c}(i), j}\left(\tilde{x}_{1}\right) \otimes \omega_{\tilde{c}(l), j}\left(\tilde{x}_{2}\right),\right.
$$

$$
\begin{aligned}
& Z(; d \tilde{c}(i), d \widetilde{c e}(i))=-\frac{1}{2 \tau} \cdot e^{-1 / 2=} \sum_{3} \frac{1}{2 \nu(\tilde{c}(i))} \\
& \left.\frac{1}{a-\tilde{c u}(i))_{j}} I_{2(\tilde{c} \tilde{\varepsilon}(i))_{j}-1}\left(\frac{1}{2 \tau}\right)-\frac{1}{a-(\tilde{c e}(i))_{j}} I_{2(\tilde{c} \tilde{e}(i))_{j}+1}\left(\frac{1}{2 \tau}\right)\right\} \\
& \cdot d \omega_{\tilde{c} \tilde{e}(i), j}\left(\tilde{x}_{1}\right) \otimes \tilde{d} \omega_{\tilde{c} \tilde{e}(i), j}\left(\tilde{r}_{2}\right) .
\end{aligned}
$$

(5.22) $\quad Z(; \operatorname{de}(i), d r \wedge \bar{e}(i))=\frac{1}{2 \tau} e^{-1 / 2=} \sum_{j} \frac{1}{2 \nu(\tilde{c e}(i))_{j}}$

$$
\begin{aligned}
& \cdot\left\{I_{\nu(\tilde{\varepsilon}(i))_{j}-1}\left(\frac{1}{2 \tau}\right)-I_{\nu(\tilde{c} e(i)\rangle_{j}+1}\left(\frac{1}{2 \tau}\right)\right\} \\
& \cdot \tilde{d} \omega_{\tilde{c} \tilde{e}(i), j}\left(\tilde{x}_{1}\right) \otimes \frac{d r_{2}}{r_{2}} \wedge \omega_{c \tilde{c}(i), j}\left(\tilde{x}_{2}\right),
\end{aligned}
$$

$$
Z(; d r, v \tilde{e}(i), d \tilde{c}(i))=\frac{1}{2 \tau} \cdot e^{-1 / 2 \tau} \sum_{3} \frac{1}{2 \nu(\tilde{c} e(i))_{3}}
$$


THE FUNDAMENTAL SOLUTIONS OF THE HEAT EQLATIONS

$$
\begin{aligned}
& \cdot\left\{I_{2(\tilde{c \tilde{e}}(u))_{j-1}}\left(-\frac{1}{2 \tau}\right)-I_{\nu(\tilde{c} \tilde{e}(i))_{j+1}}\left(\frac{1}{2 \tau}\right)\right\} \\
& \text { - } \left.\frac{d r_{1}}{r_{1}} \wedge \omega_{c \varepsilon(2), j}\left(\tilde{x}_{1}\right) \subseteq \tilde{d} \omega_{\sim}, t, \tilde{r}_{2}\right) \text {, }
\end{aligned}
$$

$$
\begin{aligned}
& Z(; \operatorname{dr} \wedge \tilde{c}(i), d r \wedge \tilde{c e}(i))=\frac{1}{2 \tau} e^{-1 / 2 \tau} \sum_{j} \frac{1}{2 \nu(\tilde{c e}(i)),} \\
& \left.\left\{a+(\tilde{c e}(i))_{j} I_{\nu(\tilde{c}(i)),-1}\left(\frac{1}{2 \tau}\right)-a^{-}(\tilde{c e}(i)), I, \ldots,\right)_{,-1}\left(\begin{array}{c}
1 \\
2 \tau
\end{array}\right)\right\} \\
& \left.\cdot \frac{d r_{1}}{r_{1}} \wedge \omega_{\tilde{c} \tilde{e}(i), j}\left(\tilde{x}_{1}\right) \otimes \frac{d r_{2}}{r_{2}}, \omega_{1,2} \ldots, \tilde{r}_{2}\right),
\end{aligned}
$$

$$
\begin{aligned}
& Z(; \operatorname{dr} \wedge \tilde{c}(i))=\frac{1}{2 \tau} e^{-1 / 2 \tau} \sum_{j} I_{\nu(\tilde{c}(i), i-1)}\left(\begin{array}{c}
1 \\
2 \tau
\end{array}\right)
\end{aligned}
$$

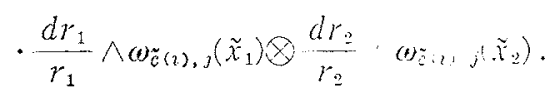

Moreover, in Cases $B(V(k)), C(V(k))$, we have

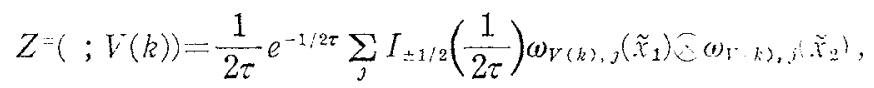

$$
\begin{aligned}
& Z=(; d r \wedge V(k))=\frac{1}{2 \tau} e^{-1 / 2 \tau} \sum_{J} I_{ \pm 1 / 2}\left(\frac{1}{2 \tau}\right) \\
& \text {. } \frac{d r_{1}}{r_{1}} \wedge \omega_{V(k), j}\left(\tilde{x}_{1}\right) \circlearrowright \frac{d r_{2}}{r_{2}}\left(\omega_{1}, k, j, \dot{x}_{2}\right),
\end{aligned}
$$

etc. Besides, by [14] III Page 172,

$$
I_{ \pm 1 / 2}\left(\frac{1}{2 \tau}\right)=\sqrt{\frac{\tau}{\pi}}\left(e^{1 / 2 \pi}+e^{-1 / 2 \tau}\right)
$$

By using these formulas, we aim to estimate the pointwise norm of $E_{C}(t ; i)$

For a while we assume $\nu>0$.

Observing the integral representation of the modified Bessel function ([14] III Page 186), we know

$$
\begin{aligned}
& \frac{1}{2 \tau} e^{-1 / 2 \pi} I_{\nu}\left(\frac{1}{2 \tau}\right) \\
& =\frac{1}{\mid 2 \pi \tau}\left\{\int_{0}^{\pi} e^{-(1-\cos s) / 2 \tau} \cos \nu s d s-\sin \nu \pi \int_{0}^{\infty} e^{-\left(1 \cdot \cos s \cdot 2 e^{-2 *} d s\right\} .}\right.
\end{aligned}
$$

Moreover we know, for any $n \in N$, 


$$
\begin{aligned}
& \int_{0}^{\pi} e^{-(1-\cos s) / 2 \tau} \cos \nu s d s-\sin \nu \pi \int_{0}^{\infty} e^{-(1+\cosh s) / 2 z} e^{-\nu s} d s \\
& =(-1)^{n} \int_{0}^{\pi}\left(\frac{\partial}{\partial s}\right)^{2 n-1}\left(e^{-(1-\cos s) / 2 \tau}\right) \frac{\sin \nu s}{\nu^{2 n-1}} d s \\
& -\sin \nu \pi \int_{0}^{\infty}\left(\frac{\partial}{\partial s}\right)^{2 n-1}\left(e^{-(1+\cosh s) / 2 \tau}\right) \frac{e^{-\nu s}}{\nu^{2 n-1}} d s \\
& =(-1)^{n} \int_{0}^{\pi}\left(\frac{\partial}{\partial S}\right)^{2 n}\left(e^{-(1-\cos s) / 2 \tau}\right) \frac{\cos \nu S}{\nu^{2 n}} d s \\
& -\sin \nu \pi \int_{0}^{\infty}\left(\frac{\partial}{\partial s}\right)^{2 n}\left(e^{-(1+\cosh s) / 2 \tau}\right) \frac{e^{-\nu s}}{\nu^{2 n}} d s .
\end{aligned}
$$

Actually, since [14. I, Page 9] yields

$$
\begin{gathered}
\left(\frac{\partial}{\partial s}\right)^{n}\left(e^{-(1-\cos s) / 2 z)=}=\sum_{k=0}^{n} \frac{\left(\frac{1}{2 \tau}\right)^{k} e^{-(1-\cos s) / 2 z}}{k !} \sum_{l=0}^{k}\left(\begin{array}{c}
k \\
l
\end{array}\right)\right. \\
\cdot(-\cos s)^{k-l}\left(\frac{\partial}{\partial s}\right)^{n}\left(\cos ^{l} s\right), \\
\left(\frac{\partial}{\partial s}\right)^{n}\left(e^{-(1+\cosh s): 2 \tau)}=\sum_{k=0}^{n} \frac{\left(\frac{1}{2 \tau}\right)^{k} e^{-(1+\cosh s) / 2=}}{k !} \sum_{l=0}^{k}\left(\begin{array}{c}
k \\
l
\end{array}\right)\right. \\
\cdot(\cosh s)^{k-l}\left(\frac{\partial}{\partial s}\right)^{n}(-\cosh s)^{l},
\end{gathered}
$$

we have

$$
\begin{aligned}
&\left.\left(\frac{\hat{\partial}}{\partial S}\right)^{2 n-1}\left(e^{-(1-\cos s) / 2 \tau}\right)\right|_{s=0}=\left.\left(\frac{\partial}{\partial s}\right)^{2 n-1}\left(e^{-(1-\cos s) / 2 \pi}\right)\right|_{s=0} \\
&=\left.\left(\frac{\partial}{\partial s}\right)^{2 n-1}\left(e^{-(1+\cosh s) / 2 \pi}\right)\right|_{s=0}=0 \\
&\left.(-1)^{n}\left(\frac{\partial}{\partial S}\right)^{2 n}\left(e^{-(1-\cos s) / 2 \tau}\right)\right|_{s=\pi}=\left.\left(\frac{\partial}{\partial S}\right)^{2 n}\left(e^{-(1+\cosh s) / 2=}\right)\right|_{s=0},
\end{aligned}
$$

and (5.30) can be verified by induction.

Now, by the formula (5.30) and the inequality ([14] III Page 186),

$$
\left|I_{2}\left(\frac{1}{2 \tau}\right)\right| \leqq \sqrt{\pi} e^{1 / 2 \cdot} \frac{(1 / 4 \tau)^{\nu}}{\Gamma(\nu+1 / 2)}
$$

we get the following lemma.

Set

$$
\begin{aligned}
\{\hat{\nu}\}_{j}= & \left\{\nu(\tilde{c}(i))_{j}\right\},\left\{\nu(\tilde{c e}(i-1))_{j}-1\right\},\left\{\nu(\tilde{c e}(z-1))_{j}+1\right\}, \\
& \left\{\nu(\tilde{c}(i-1), \imath-2)_{j}\right\},\left\{\nu(V(k))_{3},-\nu\left(V(k)^{\perp}\right)_{j}, \nu(\tilde{c}(k))_{j}\right\},
\end{aligned}
$$




$$
\text { or, }\left\{-\nu(V(k), k-1)_{j}, \nu\left(V(k)^{\llcorner}, k-1\right)_{j}, \nu(\tilde{c e}(k), k-1)_{j}\right\} \text {; }
$$

the last two sets are taken into account when the cases under considerations are Cases $B(V(k)), C(V(k))$.

LEMMA 5.1. For any $p \in \boldsymbol{N}$, there exist $K>0$ and $n \in N$ such that, for given $\tilde{\nu}_{\jmath_{0}}$,

$$
\sum_{\tilde{\nu}_{j} \geq \tilde{\Sigma}_{j}}-\frac{1}{2 \tau}-e^{-1 / 2 \tau} I_{\tilde{\nu}_{j}}\left(\frac{1}{2 \tau}\right)\left(1+\tilde{\nu}_{j}\right)^{p} \leqq K\left(\tau^{-\tilde{\nu}_{j_{0}}-1}+\tau^{-n}\right)
$$

for $t \in \boldsymbol{R}^{+}$.

Proof. First we show (5.35) when $\tilde{\nu}_{\jmath_{0}}>0$. We estimate the left hand side of (5.35) in the cases where $\tau \leqq 1$ and $\tau \geqq 1$, respectively.

First, consider the case $\tau \leqq 1$. Take $K_{1}>0$ and $n \in N$ with $\sum_{\tilde{\nu}_{j}>0} \tilde{\nu}_{j}^{-2 n}\left(1+\tilde{\nu}_{j}\right)^{p}<K_{1}$. Then, from (5.30), the left hand side of (5.35) is dominated by

$$
\begin{aligned}
& K_{1}\left\{\int_{0}^{\pi}\left|\left(\frac{\partial}{\partial s}\right)^{2 n}\left(e^{-(1-\cos s) / 2 \tau}\right)\right| d s+\int_{0}^{\infty}\left|\left(\frac{\partial}{\partial s}\right)^{2 n}\left(e^{-(1+\cosh s) / 2 \tau}\right)\right| d s\right\} \\
& \leqq K_{1} K_{2} \tau^{-2 n} \int_{0}^{\pi} e^{-(1-\cos s) / 2 \tau} d s+K_{1} e^{-1 / 2 \tau} \int_{0}^{\infty}\left|\left(\frac{\partial}{\partial s}\right)^{2 n}\left(e^{-(\cosh s) / 2 \tau}\right)\right| d s \\
& \leqq K_{1} K_{2} K_{3} \tau^{-2 n}+K_{1} K_{4} e^{-1 / 4 \tau}
\end{aligned}
$$

Second, consider the case $\tau \geqq 1$. From (5.33), the left hand side of (5.35) is dominated by

$$
K_{5} \tau^{-1} \sum_{\tilde{\nu}_{j} \geq \tilde{\nu}_{j}} \frac{(1 / 4 \tau)^{\tilde{\nu} j}}{\left(\tilde{\nu}_{j}+1 / 2\right)}\left(1+\tilde{\nu}_{j}\right)^{p} \leqq K_{5} \tau^{-\tilde{\nu}_{j_{0}}}-\sum_{\tilde{\nu}_{j}>0} \frac{\left(1+\tilde{\nu}_{j}\right)^{p}}{\Gamma\left(\tilde{\nu}_{j}+1 / 2\right)} .
$$

By applying the formula

$$
\Gamma(2 z)=\frac{2^{2 z}}{2 \sqrt{\pi}} \Gamma(z) \Gamma(z+1 / 2)
$$

as many times as we need, we know that the above is dominated by

$$
K_{5} K_{6} \tau^{-\tilde{\nu}_{j_{0}}-1} \sum_{\tilde{\nu}_{j}>0} e^{-\tilde{\nu}} \leqq K_{5} K_{6} K_{7}\left(\sum_{\tilde{\nu}_{j}>0} \tilde{\nu}_{j}^{-n}\right) \tau^{-\tilde{\nu}_{0}}{ }^{-1}
$$

Here $n \in N$ is so large that $\sum_{i j>0} \tilde{\Sigma}_{j}^{-n}<K_{8}$. Thus, as a result, the left hand side of (5.35) is dominated by

$$
K_{5} K_{6} K_{7} K_{8} \tau^{-\tilde{\nu}_{y_{0}}{ }^{-1}}
$$

Thus, by (5.36) and (5.37), the estimate (5.35) has been verified when $\tilde{\nu}_{1_{0}}>0$. Next, consider the case $(-1<) \tilde{\nu}_{\jmath_{0}} \leqq 0$. It suffices to estimate the finite sum

$$
\sum_{\tilde{\nu}_{j} \leq \tilde{\nu}_{j} \leq 0} \frac{1}{2 \tau} e^{-1 / 2 \tau} I_{\tilde{\nu}_{j}}\left(\frac{1}{2 \tau}\right)\left(1+\tilde{\nu}_{j}\right)^{p} \text {. }
$$


By the recurrence relation ([14] III Page 173), we have

$$
I_{\tilde{\nu}_{j}}\left(\frac{1}{2 \tau}\right)=4\left(\tilde{\nu}_{j}+1\right) \tau I_{\tilde{\nu}_{j}+1}\left(\frac{1}{2 \tau}\right)+I_{\tilde{\nu}_{j}+2}\left(\frac{1}{2 \tau}\right) \text {. }
$$

Hence, by applying the estimate (5.33) to the right hand side of (5.39), (5.38) is dominated by $K_{9}\left(\tau^{-\tilde{\nu}_{j}}{ }^{-1}+\tau^{-3}\right)$.

Thus the proof is complete.

Take $-1<\tilde{\nu}_{0}<0$ with $\tilde{\nu}_{j}>\tilde{\nu}_{0}$ for any $\tilde{\nu}_{j}$; see (5.34).

LEMMA 5.2. There exist $K>0$ and $n \in \boldsymbol{N}$ such that

$$
\left|E_{C}\left(t, x_{1}, x_{2} ; i\right)\right| \leqq K\left(r_{1} r_{2}\right)^{(1-m) / 2+\tilde{\nu}_{0}}\left(t^{-\tilde{\nu}_{0}-1}+t^{-n}\right) e^{-\left(r_{1}-r_{2}\right)^{2 / 4 t}}
$$

for $\left(t, x_{1}, x_{2}\right) \in \boldsymbol{R}^{+} \times C(N) \times C(N)$ with $r_{1} r_{2} \leqq 1$.

Proof. Observing (0.3), (2.18)-(2.24), and using the Sobolev lemma, there exist $K_{1}>0$ and $p \in N$ such that

$$
\begin{aligned}
& \left|\omega_{\tilde{c}(i), j}\left(\tilde{x}_{1}\right) \otimes \omega_{\tilde{c} \tilde{c}(i), j}\left(\tilde{x}_{2}\right)\right|_{\left(\left(r_{1}, \tilde{x}_{1}\right),\left(r_{2}, \tilde{x}_{2}\right)\right)} \\
& =\left(r_{1} r_{2}\right)^{-2}\left|\omega_{\tilde{c}(i), j}\left(\tilde{x}_{1}\right) \otimes \omega_{\tilde{c}(i), j}\left(\tilde{x}_{2}\right)\right|_{\left(\left(1, \tilde{x}_{1}\right),\left(1, \tilde{x}_{2}\right)\right)} \\
& =\left(r_{1} r_{2}\right)^{-2}\left|\omega_{\tilde{c} \tilde{c}(i), j}\left(\tilde{x}_{1}\right) \otimes \omega_{\tilde{c} \tilde{c}(i), j}\left(\tilde{x}_{2}\right)\right|_{\left(\tilde{x}_{1}, \tilde{x}_{2}\right)} \\
& \leqq K_{1}\left(r_{1} r_{2}\right)^{-i}\left(1+\nu(\tilde{c c}(i))_{j}\right)^{p} \\
& \left|d \omega_{c \tilde{e}(2-1), j}\left(\tilde{x}_{1}\right) \otimes \frac{d r_{2}}{r_{2}} \wedge \omega_{c \tilde{e}(i-1), j}\left(\tilde{x}_{2}\right)\right|_{\left(\left(r_{1}, \tilde{x}_{1}\right),\left(r_{2}, \tilde{x}_{2}\right)\right)} \\
& =\left(r_{1} r_{2}\right)^{-2}\left|\tilde{d} \omega_{\tilde{c} \tilde{e}(i-1), 3}\left(\tilde{x}_{1}\right) \otimes d r_{2} \wedge \omega_{\tilde{c}(i-1), j}\left(\tilde{x}_{2}\right)\right|_{\left(\left(1, \tilde{x}_{1}\right),\left(1, \tilde{x}_{2}\right)\right)} \\
& =\left(r_{1} r_{2}\right)^{-i}\left|\tilde{d} \omega_{\tilde{c} \tilde{e}(i-1), j}\left(\tilde{x}_{1}\right) \otimes \omega_{c \tilde{e}(i-1), j}\left(\tilde{x}_{2}\right)\right|_{\left(\tilde{x}_{1}, \tilde{x}_{2}\right)} \\
& \leqq K_{1}\left(r_{1} r_{2}\right)^{-2}\left(1+\nu(\tilde{c} \tilde{e}(i-1))_{j}\right)^{p},
\end{aligned}
$$

etc. Hence, by referring (5.13)-(5.27), the pointwise norm $\left|E_{C}\left(t, x_{1}, x_{2} ; i\right)\right|$ can be regarded as dominated by a finite sum of functions of the following type;

$$
K_{2}\left(r_{1} r_{2}\right)^{-(m+1) / 2} e^{-\left\langle r_{1}-r_{2}\right)^{2} / 4 t} \sum_{j} \frac{1}{2 \tau} e^{-1 / 2 \tau} I_{\tilde{\nu}_{j}}\left(\frac{1}{2 \tau}\right)\left(1+\tilde{\nu}_{j}\right)^{p} .
$$

Here, we are setting $\tau=t /\left(r_{1} r_{2}\right)$. The $\left\{\tilde{\nu}_{j}\right\}$ 's are (5.34). Moreover, by Lemma 5.1 , for some $K_{2}>0$ and $n \in N,(5.41)$ is dominated by

$$
\begin{aligned}
& K_{1} K_{2}\left(r_{1} r_{2}\right)^{-(m+1) / 2} e^{-\left(r_{1}-r_{2}\right)^{2} / 4 t}\left(\frac{\left(r_{1} r_{2}\right)^{\tilde{\nu}_{0}+1}}{t^{\tilde{\nu}_{0}+1}}+\frac{\left(r_{1} r_{2}\right)^{n}}{t^{n}}\right) \\
\leqq & 2 K_{1} K_{2}\left(r_{1} r_{2}\right)^{(1-m) / 2+\tilde{\nu}_{0}} 0\left(t^{-\tilde{\nu}_{0}-1}+t^{-n}\right) e^{-\left(r_{1}-r_{2}\right)^{2} / 4 t} .
\end{aligned}
$$

Thus the proof is complete.

Moreover, by the argument similar to the proof of Lemma 5.1, we know that, for any $l \in N$, there exist $K>0$ and $n \in N$ such that 


$$
\left|\left(\frac{\partial}{\partial t}\right)^{l} E_{C}\left(t, x_{1}, x_{2} ; i\right)\right| \leqq K\left(r_{1} r_{2}\right)^{(1-m) ; 2+\Sigma_{0}\left(t^{-z_{0}-l-1}+t^{-n}\right)} e^{-\left(r_{1}-r_{2}\right)^{2 / 4 t}}
$$

for $\left(t, x_{1}, x_{2}\right) \in R^{+} \times C(N) \times C(N)$ with $r_{1} r_{2} \leqq 1$.

Besides, let us take $\varepsilon^{\prime \prime}>0$ such that

$$
-1-2 \widetilde{\Sigma}_{0}<\varepsilon^{\prime \prime}<1 \text {. }
$$

(This number $\varepsilon^{\prime \prime}$ is just the number $\varepsilon^{\prime \prime}$ which was referred in the property (II).) Moreover, let $\varphi(r)$ be a positive smooth function with $\varphi(r)=r^{-\left(m+\varepsilon^{*}\right) / 2}$ for $r$, small. Then, for any $\varepsilon_{0}>0$ and any $P=P\left(\frac{\partial}{\partial r_{1}}, d_{x_{1}}, \delta_{x_{1}}\right)$, that is, any polynomial of $\frac{\partial}{\partial r_{1}}, d_{x_{1}}$ and $\delta_{x_{1}}$, there exist $K>0$ and $n \in N$ such that

$$
\left|P E_{C}\left(t, x_{1}, x_{2} ; i\right)\right| \leqq K \varphi\left(r_{2}\right)\left(1+t^{-n}\right) e^{-\left(r_{1}-r_{2}\right) 2^{2} / t}
$$

for $\left(t, x_{1}, x_{2}\right) \in \boldsymbol{R}^{+} \times C_{\varepsilon_{0}, 1}(N) \times C(N)$ with $r_{1} r_{2} \leqq 1$. Since our $P$ does not involve $d_{x_{2}}, \delta_{x_{2}}$ and we have cut off the neighborhood of the singular point with respect to $x_{1},(5.44)$ can be verified by the argument similar to the proof of $(5.40)$.

Further, by the similar argument, we can easily show that, for any $0<\varepsilon_{0}<\varepsilon_{1}$ and any $P=P\left(\frac{\partial}{\partial r_{1}}, \frac{\partial}{\partial r_{2}}, d_{x_{j}}, \delta_{x_{j}}, \frac{\partial}{\partial t}\right)$ of order $\leqq l$, there exist $K=K\left(\varepsilon_{0}, \varepsilon_{1}, l\right)>0$ and $n=n\left(\varepsilon_{0}, \varepsilon_{1}, l\right) \in \boldsymbol{N}$ such that

$$
\left|P E_{C}\left(t, x_{1}, x_{2} ; i\right)\right| \leqq K\left(1+t^{-u}\right) e^{-\left(r_{1}-r_{2}\right) 2 / 4 t}
$$

for $\left(t, x_{1}, x_{2}\right) \subseteq \boldsymbol{R}^{+} \times C_{s_{0}, \varepsilon_{1}}(N) \times C_{\varepsilon_{0}, \varepsilon_{1}}(N)$.

Hence $E_{C}\left(t, x_{1}, x_{2} ; i\right)$ is smooth on $\boldsymbol{R}^{+} \times C(N) \times C(N)$. Moreover, as is easily observed, it satisfies the boundary condition with respect to both $x_{1}$ and $x_{2}$. We may remark that it has already been shown that, in the case where the ideal boundary condition is concerned, $E_{C}$ satisfies also the ideal one.

We will now construct $F\left(t, x_{1}, x_{2} ; i\right)$ which has the properties (I) and (II), and verify the remained condition (5.5). we get

Applying $\frac{\partial}{\partial t}+1_{x_{1}}$ to both sides of $(5.3)$ and assuming that $(5.5)$ is valid,

$$
\begin{aligned}
F\left(t, x_{1}, x_{2} ; i\right)=G\left(t, x_{1}, x_{2} ; i\right) \\
-\int_{0}^{t} d t_{1} \int_{X} G\left(t-t_{1}, x_{1}, x_{3} ; i\right) \wedge *_{x_{3}} F\left(t_{1}, x_{3}, x_{2} ; i\right),
\end{aligned}
$$

where we set

$$
G\left(t, x_{1}, x_{2} ; i\right)=\left(-\frac{\partial}{\partial t}+\Delta_{x_{1}}\right) \tilde{E}\left(t, x_{1}, x_{2} ; i\right) .
$$

Hence, formally, $F\left(t, x_{1}, x_{2} ; i\right)$ is the solution of the integral equation (5.46), and, as is well known, it can be written as the following sum, namely, the Levi's 
sum.

$$
F\left(t, x_{1}, x_{2} ; i\right)=\sum_{j} F_{j}\left(t, x_{1}, x_{2} ; i\right)
$$

$F_{0}\left(t, x_{1}, x_{2} ; i\right)=G\left(t, x_{1}, x_{2} ; i\right)$

$$
\begin{aligned}
& F_{1}\left(t, x_{1}, x_{2} ; i\right)=-\int_{0}^{t} d t_{1} \int_{X} G\left(t-t_{1}, x_{1}, x_{3} ; i\right) \wedge *_{x_{3}} F_{0}\left(t_{1}, x_{3}, x_{2} ; i\right) \\
& \quad \vdots \\
& F_{j+1}\left(t, x_{1}, x_{2} ; i\right)=-\int_{0}^{t} d t_{1} \int_{X} G\left(t-t_{1}, x_{1}, x_{3} ; i\right) \wedge *_{x_{3}} F_{j}\left(t_{1}, x_{3}, x_{2} ; i\right)
\end{aligned}
$$

Therefore, for our purposes (involving the purpose of the verification of (5.5)), it suffices to show that the Levi's sum (5.48) has the properties (I) and (II).

Now let us apply $\frac{\partial}{\partial t}+\Delta_{x_{1}}$ to the first and the second terms of the right hand side of (5.2) and let $G_{C}\left(t, x_{1}, x_{2} ; i\right)$ and $G_{M}\left(t, x_{1}, x_{2} ; i\right)$ be the two terms thus obtained. Moreover, according to the decomposition of $E_{C}(; i)$, let us decompose $G_{C}(; i)$ into $G_{C}(; \widetilde{c}(i))+G_{C}(; d \tilde{c e}(i-1)+d r \wedge \tilde{c e}(i-1))+G_{C}(; d r \wedge \tilde{c}(i))$ (in Case $A$ ), etc. Then, by (2.7), we have

$$
\begin{aligned}
& G_{C}(; \tilde{c}(i))=-\left(\Psi_{C}^{\prime \prime}\left(r_{1}\right)+2 \Psi_{C}^{\prime}\left(r_{1}\right) \frac{\partial}{\partial r_{1}}+(m-2 i) r_{1}^{-1} \Psi_{C}^{\prime}\left(r_{1}\right)\right) E_{C}(; \tilde{c c}(i)) \Phi_{C}\left(x_{2}\right), \\
& G_{C}(; d \tilde{c e}(i-1)+d r \wedge \tilde{c e}(i-1)) \\
&=-\left(\Psi_{C}^{\prime \prime}\left(r_{1}\right)+2 \Psi_{C}^{\prime}\left(r_{1}\right) \frac{\partial}{\partial r_{1}}+(m-2 i) r_{1}^{-1} \Psi_{C}^{\prime}\left(r_{1}\right)\right) \\
& \cdot E_{C}(; d \tilde{c e}(i-1), d \tilde{c}(i-1)+d r \wedge \tilde{c e}(i-1)) \Phi_{C}\left(x_{2}\right) \\
&-\left(\Psi_{C}^{\prime \prime}\left(r_{1}\right)+2 \Psi_{C}^{\prime}\left(r_{1}\right) \frac{\partial}{\partial r_{1}}+(m-2(i-1)) r_{1}^{-1} \Psi_{C}^{\prime}\left(r_{1}\right)\right) \\
& \cdot E_{C}(; d r \wedge \tilde{c e}(i-1), d \tilde{c e}(i-1)+d r \wedge \tilde{c e}(i-1)) \Phi_{C}\left(x_{2}\right),
\end{aligned}
$$

etc. Hence $G_{C}\left(t, x_{1}, x_{2} ; i\right)$ has support on $\boldsymbol{R}^{+} \times C_{5 \varepsilon, 6 \varepsilon}(N) \times C_{0,4 \varepsilon}(N)$. Similarly, $G_{M}\left(t, x_{1}, x_{2} ; i\right)$ has support on $\boldsymbol{R}^{+} \times C_{\varepsilon, 2 \varepsilon}(N) \times X_{3 s}$.

Paying attention to the fact that the supports of $G_{C}$ and $G_{M}$ are thus apart from the diagonal set with respect to $x_{1}$ and $x_{2}$, we can easily show by (5.45) and the similar estimate for $E_{M}$ that, for any $\varepsilon_{0}>0$ and any $P=P\left(\frac{\partial}{\partial r_{1}}, \frac{\partial}{\partial r_{2}}\right.$, $\left.d_{x_{j}}, \delta_{x_{j}}, \frac{\partial}{\partial t}\right)$ of order $\leqq l$, there exist $K=K\left(\varepsilon_{0}, l\right)>0$ and $\delta=\delta\left(\varepsilon_{0}, l\right)>0$ such that

$$
\left|P G\left(t, x_{1}, x_{2} ; i\right)\right| \leqq K e^{-\tilde{\delta} / t}
$$

for $\left(t, x_{1}, x_{2}\right) \in \boldsymbol{R}^{+} \times X_{\varepsilon_{0}} \times X_{\varepsilon_{0}}$. Hence, we have the following inequalities on $\boldsymbol{R}^{+} \times X_{\varepsilon_{0}} \times X_{s_{0}}$.

$$
\left|P F_{0}\left(t, x_{1}, x_{2} ; i\right)\right| \leqq K e^{-\delta / t},
$$




$$
\begin{aligned}
\left|P F_{1}\left(t, x_{1}, x_{2} ; i\right)\right| & \leqq K K_{1} \operatorname{vol}(N) \int_{0}^{t} d t_{1} \int_{\varepsilon}^{6 \varepsilon} r_{3}^{m} e^{-\delta\left(1 /\left(t-t_{1}\right)+1 / t_{1}\right)} d r_{3} \\
& \leqq K \tilde{K} \int_{0}^{t} e^{-\delta / t} d t \leqq K \tilde{K} t e^{-\delta / t}
\end{aligned}
$$

Here, we set

$$
\tilde{K}=K_{1} \operatorname{vol}(N) \frac{1}{m+1}\left\{\left((6 \varepsilon)^{m+1}-\varepsilon^{m+1}\right) .\right.
$$

We may take $\varepsilon>0$ so small that $\tilde{K}<1$. In general, we have the inequality

$$
\left|P F_{j}\left(t, x_{1}, x_{2} ; i\right)\right| \leqq K \tilde{K}^{j} \frac{t^{j}}{\jmath !} e^{-\delta / t}
$$

for $\left(t, x_{1}, x_{2}\right) \in \boldsymbol{R}^{+} \times X_{\varepsilon_{0}} \times X_{\varepsilon_{0}}$. Hence, any termwise derivatives of the infinite series (5.48) converge absolutely on $\boldsymbol{R}^{+} \times X_{\varepsilon_{0}} \times X_{\varepsilon_{0}}$ and, moreover,

$$
\left|P F\left(t, x_{1}, x_{2} ; i\right)\right| \leqq K e^{-\delta / t},
$$

for $t$, small.

By the above fact and the fact that $F$ has a compact support with respect to $x_{1}$, we know that $F$ has really the property (I). Moreover, by (5.44) and the similar estimate for $E_{M}$, we know that $F$ has the property (II).

Thus, our purpose of reconstructing the heat kernel has just been accomplished.

Now, by the uniqueness of the heat kernel ([10] Lemma 1), we have

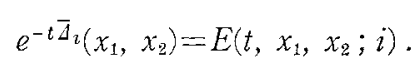

Moreover, we have

$$
\begin{aligned}
& e^{-t \bar{d}_{i}}\left(x_{1}, x_{2}\right)=E_{C}\left(t, x_{1}, x_{2} ; i\right) \div O\left(e^{-\delta / t}\right) \quad \text { on } C_{2 \varepsilon, g_{\xi}}(N) \times C_{2 \varepsilon, 3 s}(N) \text {, } \\
& e^{-t \bar{d}_{i}}\left(x_{1}, x_{2}\right)=E_{M}\left(t, x_{1}, x_{2} ; i\right)+O\left(e^{-\delta / t}\right) \quad \text { on } C_{4 \varepsilon, \bar{s} \varepsilon}(N) \times C_{t \varepsilon, \bar{s} \varepsilon}(N) \text {. }
\end{aligned}
$$

Here, $O\left(e^{-\delta / t}\right), \delta>0$, is a term any derivatives of which decrease exponentially when $t \downarrow 0$. For showing (5.55) and (5.56), it suffices to prove

$$
\int_{0}^{t} d t_{1} \int_{X} \tilde{E}\left(t-t_{1}, x_{1}, x_{3} ; i\right) \wedge *_{x_{3}} F\left(t_{1}, x_{3}, x_{2} ; i\right)=O\left(e^{-\grave{o} / t}\right)
$$

on respective subspaces. We can assume that $F\left(t_{1}, x_{3}, x_{2} ; i\right)$ has a support on $\boldsymbol{R}^{+} \times\left\langle C_{\varepsilon, 2 \varepsilon-\varepsilon_{0}}(N) \cup C_{5 s+\varepsilon_{0}, \varepsilon_{s}}(N)\right) \times X$ with $\varepsilon_{0}>0$, small. Therefore, about (5.57), the integral over $X$ is practically the integral over $C_{\varepsilon, 2 \varepsilon-\varepsilon_{0}}(N) \cup C_{5 \varepsilon+\varepsilon_{0}, 6 \varepsilon}(N)$. Hence (5.57) can be verified by observing (5.53), (5.44) and the similar estimate for $E_{M}$.

By (5.55), (5.56) and, again, by the uniqueness of the heat kernel, we can get the following valuable lemma.

Before stating our lemma, let us make some remarks. To avoid complicating the explanation, we may assume first that our $X$ can be described as 
$X=C_{u, u+s_{1}}(N) \cup X_{u+s_{1}}$ with $\varepsilon_{1}>0$, sufficiently small. (In other words, we may formally substitute $u+\varepsilon_{1}$ for $u$.) Second, we have took a sequence $\varepsilon^{\prime}<\varepsilon<2 \varepsilon<$ $\cdots<6 \varepsilon<u+\varepsilon_{1}$ and constructed the heat kernel $E$, but there is no necessity to start our construction with specifying a sequence so definitely. More freely, we can construct $E$ from a sequence

$$
\varepsilon^{\prime}<r_{1}<r_{2}<\cdots<r_{j 6}<u+\varepsilon_{1}
$$

with $r_{i}-r_{1}$, sufficiently small.

Now take $\varepsilon^{\prime}<\varepsilon_{0}<u$. Then we have

LEMMA 5.3. Keeping the distance between the two pornts $x_{1}$ and $x_{2}$ of $C_{s_{0}, u}(N)$ suificiently short, we have

$$
\begin{aligned}
e^{\left.-t \bar{J}_{i\left(x_{1}\right.}, x_{2}\right)} & =E_{C}\left(t, x_{1}, x_{2} ; i\right)+O\left(e^{-\delta / t}\right) \\
& -E_{M}\left(t, x_{1}, x_{2} ; i\right)+O\left(e^{-\tilde{\delta} / t}\right) .
\end{aligned}
$$

Proof. If we construct the heat kernel $E$ from the sequence (5.58), then (5.55) and (5.56) can be rewritten as follows;

$$
e^{-l \hat{J}_{i}}\left(x_{1}, x_{2}\right)= \begin{cases}E_{C}\left(t, x_{1}, x_{2} ; i\right)+O\left(e^{-\delta / t}\right) & \text { on } C_{\eta_{2}, \eta_{3}}(N) \times C_{\eta_{2}, \eta_{3}}(N) \\ E_{. Y}\left(t, x_{1}, x_{2} ; i\right)+O\left(e^{-\hat{o} / t}\right) & \text { on } C_{\eta_{1}, \eta_{j}}(N) \times C_{\eta_{1}, \eta_{5}}(N) .\end{cases}
$$

Therefore, by changing (5.58) gradually, (5.59) can be verified.

We can now investigate the trace of $(5.1)$.

First we have

$$
\begin{aligned}
& \int_{C_{0, z}(x)} \operatorname{Tr} \int_{0}^{t} d t_{1} \int_{X} \tilde{E}\left(t-t_{1}, x, x_{3} ; i\right) \wedge *_{x_{3}} F\left(t_{1}, x_{3}, x ; i\right)=O\left(e^{-\delta / t}\right), \\
& \int_{X_{0 z}} \operatorname{Tr} \int_{0}^{t} d t_{1} \int_{X} \tilde{E}\left(t-t_{1}, x, x_{3} ; i\right) \wedge *_{x_{3}} F\left(t_{1}, x_{3}, x ; i\right)=O\left(e^{-\hat{o} / t}\right) .
\end{aligned}
$$

Here $O\left(e^{-\delta / t}\right), \delta>0$, is a function of $t$ any derivatives of which decrease exponentially when $t ! 0$. The left hand sides of (5.61) and (5.62) are the integrals of the pointwise traces over $C_{0, s}(N)$ and $X_{6 \varepsilon}$, respectively. These are easily verified by (5.53) and (5.42) and the similar estimate for $E_{Y}$, and by the fact that the integral over $X$ is practically an integral over $C_{\varepsilon+\varepsilon_{0}, 2 \varepsilon}(N) \cup C_{5 \varepsilon, 6 \varepsilon-\varepsilon_{0}}(N)$ with $\varepsilon_{0}>0$, small. We may remark that (5.6) and (5.40) guarantee the integrability of the left hand side of (5.61).

LEMMA 5.4. Given $\varepsilon^{\prime}<\varepsilon_{0} \leqq \varepsilon_{1}<u$,

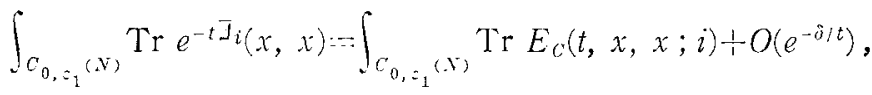

$$
\begin{aligned}
& \int_{X_{\Sigma_{0}}} \operatorname{Tr} e^{-t \tilde{J}}(x, x)=\int_{X_{\Sigma_{0}}} \operatorname{Tr} E_{M}(t, x, x ; i)+O\left(e^{-\tilde{j} / t}\right) .
\end{aligned}
$$


Proof. The integrabilities of both sides of (5.63) are guaranteed by (5.40) and (5.54). The lemma can be verified similarly to the proof of Lemma 5.3 by using (5.61) and (5.62).

Hence, first, for investigating the asymptotic behavior of the left hand side of (5.64) when $t ! 0$, it suffices to investigate $\int_{x_{\varepsilon_{0}}} \operatorname{Tr} E_{M}(t, x, x ; i)$. And this is well known $([11],[17] \S 5)$. That is, there exist the smooth double forms on $M$, $a_{j / 2}(x)=a_{j / 2}(x ; i), j=0,1,2, \cdots$ with $a_{y / 2}(x)=0$ if $\jmath$ is odd, and smooth forms on $\partial M, b_{j / 2}(x)=b_{j / 2}(x ; i), j=0,1,2, \cdots$, such that, if we set

$$
{ }_{i} a_{j / 2}^{\langle\xi\rangle}=\int_{X_{\tau}} \operatorname{Tr} a_{j / 2}(x)+\int_{\widehat{o} \hat{X}_{z}} \operatorname{Tr} b_{j / 2}(x), \quad y=0,1,2, \cdots,
$$

then we have

$$
\int_{x_{i 0}} \operatorname{Tr} e^{-i J_{2}}(x, x) \sim \sum_{j=0}^{\infty} i a_{j, 2}^{\left\langle\delta_{0} t^{\prime}\right.} t^{-(m+1) \cdot z-j ! y}, \quad t_{>} 0
$$

Besides, if we set, for $r$ with $\varepsilon_{0} \leqq r \leqq u$,

$$
{ }_{2}^{r} a_{j / 2}=r_{i} \tilde{a}_{3 / 2} d r=\int_{(r, v)} \operatorname{Tr} a_{j / 2}(r, \tilde{x}) \div \int_{\partial\langle(r, v)} \operatorname{Tr} b_{j, 2}(r, \tilde{x}),
$$

we have

$$
\begin{aligned}
& { }_{i}^{r} \tilde{a}_{j i,}=\left(\frac{r}{u}\right)^{m-3}{ }_{i}^{u} \tilde{a}_{j i,},
\end{aligned}
$$

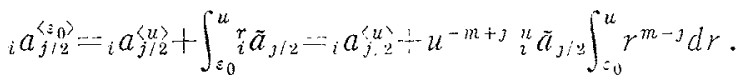

In the following, we will omit the subscript " $i$ ", $i$.e. we use the notations $a_{j,}$, ${ }^{r} \tilde{a}_{j / 2}$, etc. instead of ${ }_{i} a_{j / 2}^{\langle\delta\rangle},{ }_{i}^{r} \tilde{a}_{j / 2}$, etc.

Now, we know from $(5.65)-(5.69)$ that

$$
a_{j / 2}^{\left\langle\varepsilon_{0}\right\rangle=} \begin{cases}a_{j / 2}^{\{\zeta\rangle}+{ }^{u} \tilde{a}_{j / 2}\left(\frac{u}{m+1-1}, \frac{u^{-n+\jmath} \varepsilon_{0}^{m+1-3}}{m+1-j}\right) ; & \jmath \div n \div 1, \\ a_{(m+1) / 2}^{\{u\rangle}+{ }^{u} \tilde{a}_{j / 2} u \log \frac{u}{\varepsilon_{0}} & , \quad \jmath=n \div 1 .\end{cases}
$$

Let us explain (5.68) roughly. Let us define

$$
\text { ss : } C(N) \rightarrow C(N)
$$

to be the map $(r, \tilde{x}) \rightarrow(s r, \tilde{x})$. Then, by $(2.1)$ and $(2.4)$, we have the formulas;

$$
\begin{aligned}
& \iota_{s}^{*} A=s^{-2} t_{s}^{*}, \\
& \iota_{s}^{*}=s^{m+1-2 z_{*}^{*}} \quad \text { on } i \text {-forms. }
\end{aligned}
$$

Therefore, by the uniqueness of the (pointwise) asymptotic expansion of the heat 
kernel, we have

$$
{ }^{r} \tilde{a}_{j / 2}=s^{-m+j s r} \tilde{a}_{j / 2},
$$

if $\varepsilon_{0} \leqq r \leqq u$ and $\varepsilon_{0} \leqq s r \leqq u$. Hence, by setting $s r=u$, we get (5.68).

By the way, let $\operatorname{Tr}_{(r, r)} E_{M}(t ; i)$ be the trace of $E_{M}(t,(r, \tilde{x}),(r, \tilde{x}) ; i)$ with respect to the inner product $(2.18)$ with $s=r$. Then we have

$$
\operatorname{Tr}_{(r, r)} E_{M}(t ; i) \sim \sum_{j=0}^{r} \tilde{a}_{3 / 2} t^{-(m+1) / 2+j / 2}, \quad t \downarrow 0 .
$$

This expansion plays an important role in studying the asymptotic expansion of (5.63).

Now, we will expand (5.63). To do so, it is necessary to collect the properties of $Z(; i)$. Let us begin with it.

Let $\operatorname{Tr}_{(r, r)} Z(t ; i)$ be the trace of $Z(t,(r, \tilde{x}),(r, \tilde{x}) ; i)$ with respect to the inner product (2.18) with $s=r$. In particular, set $\operatorname{Tr}_{N} Z(t ; i)=\operatorname{Tr}_{(1,1)} Z(t ; i)$. Then, by Theorem 3.1 and $(5.13)-(5.28)$, we have

\section{LEMMA 5.5.}

(1) In general,

$$
\operatorname{Tr}_{(r, r)} Z(t ; i)=r^{m-22} \operatorname{Tr}_{N} Z\left(\frac{t}{r^{2}} ; \imath\right) .
$$

(2) In Case A,

$$
\begin{aligned}
& \operatorname{Tr}_{N} Z(t ; i)=\frac{1}{2 t} e^{-1 / 2 t}\left\{\sum_{j} I_{\nu(\tilde{c} \tilde{\sigma}(2))_{j}}\left(\frac{1}{2 t}\right)+2 \sum_{j} I_{\nu(\tilde{\varepsilon}(i-1))_{j}}^{\prime}\left(\frac{1}{2 t}\right)\right. \\
& \left.+\sum_{j} I_{\nu(\tilde{c}(i-1), 2-2)}\left(\frac{1}{2 t}\right)\right\} \text {. }
\end{aligned}
$$

(3) In Case $B(V(k))$,

$$
\begin{aligned}
\operatorname{Tr}_{N} Z(t ; i)=\frac{1}{2} t e^{-1 / 2 t}\{ & \operatorname{dim} V(k) I_{1 / 2}\left(\frac{1}{2 t}\right)+\operatorname{dim} V(k)^{\perp} I_{-1 / 2}\left(\frac{1}{2 t}\right) \\
& +\sum_{\jmath} I_{\nu(\tilde{C} \tilde{\theta}(k))_{j}}\left(-\frac{1}{2 t}\right)+2 \sum_{\jmath} I_{\nu(\tilde{c}(k-1))_{j}}^{\prime}\left(\frac{1}{2 t}\right) \\
& \left.+\sum_{J} I_{\nu(\hat{c}(k-1), k-2)}\left(\frac{1}{2 t}\right)\right\} .
\end{aligned}
$$

(4) In Case $C(V(k))$,

(5 77)

$$
\begin{aligned}
\operatorname{Tr}_{N} Z(t ; i)=\frac{1}{2 t} e^{-1 / 2 t}\left\{\sum_{\jmath} I_{\nu\langle\tilde{c} \tilde{c}(k+1)\rangle_{j}}\left(\frac{1}{2 t}\right)+2 \sum_{j} I_{\nu(\tilde{e}(k))_{j}}\left(\frac{1}{2 t}\right)\right. \\
+\operatorname{dim} V(k) I_{-1 / 2}\left(\frac{1}{2 t}\right)+\operatorname{dim} V(k)^{1} I_{1 / 2}\left(\frac{1}{2 t}\right) \\
\left.+\sum_{i} I_{\nu(\tilde{e}(k), k-1)_{j}}\left(\frac{1}{2 t}\right)\right\} .
\end{aligned}
$$


By (5.18), (5.59), (5.73) and (5.74), we have

$$
\begin{aligned}
\operatorname{Tr}_{N} Z\left(\frac{t}{r^{2}} ; \imath\right)= & r \operatorname{Tr}_{(r, r)} E_{M}(t ; i)+O\left(e^{-\delta / t}\right) \\
& \sim \sum_{j=0}^{\infty} r^{r} \tilde{a}_{j / 2} t^{-(m+1) / 2+j / 2}, \quad t \downarrow 0 .
\end{aligned}
$$

Therefore we have

$$
\operatorname{Tr}_{N} Z(t ; i) \sim \sum_{j=0}^{\infty} \alpha_{j / 2} t^{-(m+1) / 2+j / 2}, \quad t \downarrow 0
$$

with

$$
\alpha_{j / 2}={ }_{\imath} \alpha_{j / 2}=\frac{{ }^{r} \tilde{a}_{j / 2}}{r^{m-\jmath}} .
$$

We may remark that the $\alpha_{j / 2}$ 's are independent of $r$. On the other hand, by applying the method similar to the proof of (5.35) when $\tau \geqq 1$ to $(5.75)-(5.77)$, we can show that

$$
\operatorname{Tr}_{N} Z(t ; i) \sim K t^{\nu_{0}}, \quad t \uparrow \infty,
$$

where $\nu_{0}<0$. The asymptotic orders of the derivatives of $\operatorname{Tr}_{N} Z(t ; i)$ when $t \uparrow \infty$ is, of course, lower than (5.81).

Now, we will return to the study of (5.63). By (5.18), we have

$$
\begin{aligned}
\int_{C_{0, \varepsilon_{1}}(N)} & \operatorname{Tr} E_{C}(t, x, x ; i)=\int_{C_{0, \varepsilon_{1}}(N)} r^{2 \tau-(m+1)} \operatorname{Tr} Z(t,(r, \tilde{x}),(r, \tilde{x}) ; i) \\
= & \int_{0}^{\varepsilon_{1}} r^{2 \imath-(m+1)} \operatorname{Tr}_{(r, r)} Z(t ; i) d r=\int_{0}^{\varepsilon_{1}} r^{-1} \operatorname{Tr}_{N} Z\left(\frac{t}{r^{2}} ; \imath\right) d r \\
= & \frac{1}{2} \int_{t / \varepsilon_{1}^{2}}^{\infty} \tau^{-1} \operatorname{Tr}_{N} Z(\tau ; i) d \tau .
\end{aligned}
$$

Hence, if we set

$$
\tilde{Z}(t ; i)= \begin{cases}\operatorname{Tr}_{N} Z(t ; i)--\sum_{j=0}^{m-1} \alpha_{j / 2} t^{-(m+1) / 2+\jmath / 2} ; & t \leqq 1 \\ \operatorname{Tr}_{N} Z(t ; i)-\sum_{j=0}^{m} \alpha_{j / 2} t^{-(m+1) / 2+\jmath / 2} ; & t>1,\end{cases}
$$

then (5.82) equals to

$$
\begin{aligned}
& \frac{1}{2} \sum_{j=0}^{m} \alpha_{j / 2} \int_{t / \varepsilon_{1}^{2}}^{\infty} \tau^{-1-(m+1) / 2+j / 2} d \tau+\frac{1}{2} \alpha_{(m+1) / 2} \int_{t / \varepsilon_{1}^{2}}^{1} \tau^{-1} d \tau \\
& \quad+\frac{1}{2} \int_{0}^{\infty} \tau^{-1} \tilde{Z}(\tau ; i) d \tau-\frac{1}{2} \int_{0}^{t / \varepsilon_{1}^{2}} \tau^{-1} \tilde{Z}(\tau ; i) d \tau \\
& =\sum_{j=0}^{m} \frac{\varepsilon_{1}^{m+1-\jmath}}{m+1-j} \alpha_{j / 2} t^{-(m+1) / 2+j / 2}-\frac{1}{2} \alpha_{(m+1) / 2} \log t
\end{aligned}
$$




$$
+\left\{\alpha_{(m+1) ; 2} \log \varepsilon_{1}-\frac{1}{2} \int_{0}^{\infty} \tau^{-1} \tilde{Z}(\tau ; i) d \tau\right\}-\frac{1}{2} \int_{0}^{l / s_{1}^{2}} \tau^{-1} \tilde{Z}(\tau ; i) d \tau
$$

We note that $\tau^{-1} \tilde{Z}(\tau ; i)$ is integrable over $\boldsymbol{R}^{+}$because of (5.79), (5.81) and (5.83). Moreover, observing (5.79), we have

$$
-\frac{1}{2} \int_{0}^{t / \varepsilon_{1}^{2}} \tau^{-1} \widetilde{Z}(\tau ; i) d \tau \sim \sum_{\jmath>m+1} \frac{\varepsilon_{1}^{m+1-\jmath}}{m+1-\jmath} \alpha_{\jmath, 2} t^{-(m+1) / 2+j / 2}
$$

when $t \downarrow 0$. Thus we get the asymptotic expansion of (5.63).

Now we observe (5.66), (5.82), (5.84) and (5.85). In particular, by setting $\varepsilon_{1}=\varepsilon_{1}$, we can get the asymptotic expansion of (5.1). That is, if we set

$$
\begin{aligned}
{ }_{i} A_{j / 2}=\left\{\begin{array}{cl}
{ }_{i} a_{j / 2}^{\langle u\rangle} m+\frac{u}{m+1},{ }_{l}^{u} \tilde{a}_{j / 2} \\
{ }_{i} a_{(m+1) / 2}^{\langle u\rangle}+{ }_{\imath}^{u} \tilde{a}_{(m+1) / 2} u \log u+\frac{1}{2} \int_{0}^{\infty} t^{-1} \tilde{Z}(t ; i) d t ; & j=m+1,
\end{array}\right. \\
{ }_{2} A=-\frac{1}{2} u_{\imath}^{u} \tilde{a}_{(m+1) / 2}=-\frac{1}{2}{ }_{\imath} \alpha_{(m+1) / 2},
\end{aligned}
$$

which are, of course, independent of $u$, then we have

THEOREM 5.6.

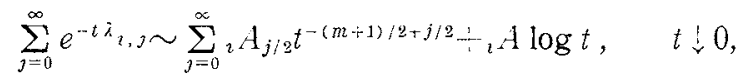

$$
\begin{aligned}
& { }_{0} A_{0}=\frac{\operatorname{vol}(X)}{(4 \pi)^{(m+1) / 2}} .
\end{aligned}
$$

Besides, if we set

$$
\begin{gathered}
\zeta_{i}(s)=\sum_{\lambda, j>0} \lambda_{i, j}^{-s}, \\
N_{i}(\lambda)=\#\left\{\lambda_{i, j}\left\{\lambda_{2, j}<\lambda\right\},\right.
\end{gathered}
$$

then, by an usual argument, we get

COROLLARY 5.7.

(1) The zeta function $\zeta_{i}(s)$ can be extended to a meromorphac function with stmple poles at points $(n+1) / 2-j / 2$ which are not negative integers. The residue of the pole at the point $(m+-1) / 2-j / 2 \neq 0$, is equal to $\Gamma((m+1) / 2-j / 2)^{-1}{ }_{2} A_{j / 2}$. The residue of the pole at the point $(m+1) / 2-j / 2=0$, that $2 s$, the origin, is equal to $-A=\frac{1}{2}{ }_{i} \alpha_{(m+1) / 2}$.

(2)

$$
N_{\imath}(\lambda) \sim \frac{{ }_{l} A_{0} \lambda^{(m+1) / 2}}{\Gamma((m+1) / 2+1)}, \quad \lambda \hat{i} \infty
$$


In particular,

$$
N_{0}(\lambda) \sim \frac{\operatorname{vol}(X) \lambda^{(m+1) / 2}}{(4 \pi)^{(m+1) / 2} \Gamma((m+1) / 2+1)}, \quad \lambda \uparrow \infty .
$$

Proof. Set $b_{i}=\operatorname{dim} \mathscr{H}^{i}(X)$ and

$$
e^{t \underline{\Delta}_{i}}=e^{-t \bar{d}_{i}}-\sum_{\lambda, j=0} e^{-t \lambda} i, \lambda \varphi_{i, j} \otimes \varphi_{i, j} .
$$

Take $N \ni n>\frac{m+1}{2}$. Then, if $\operatorname{Re}(s)>\frac{m+1}{2}$,

$$
\begin{aligned}
& \zeta_{i}(s)=\frac{1}{\Gamma(s)} \int_{0}^{\infty} t^{s-1} \operatorname{Tr} e^{-t} \underline{d}_{\imath} d t \\
& =\frac{1}{\Gamma(s)}\left\{\int_{s}^{\infty} t^{s-1} \operatorname{Tr} e^{-t \underline{\underline{J}}} \imath d t+\int_{0}^{s} t^{s-1} \mu_{n}(t) d t\right. \\
& \left.\frac{b_{i}}{s} \varepsilon^{s}+\sum_{j=0}^{2 n} \frac{{ }_{i} A_{j / 2} \varepsilon^{s-(m+1) / 2+j / 2}}{s-(m+1) / 2+j / 2}+{ }_{1} A\left(\frac{\varepsilon^{s} \log \varepsilon}{s}-\frac{\varepsilon^{s}}{s^{2}}\right)\right\},
\end{aligned}
$$

where $\mu_{n}(t)=O\left(t^{-(m+1) / 2+n+1 / 2}\right), t \downarrow 0$. Here, $\frac{1}{\Gamma(s)} \int_{0}^{\infty} t^{s-1} \operatorname{Tr} e^{-t} \underline{I}_{2} d t$ can be extended to a holomorphic function on $C$ and $\frac{1}{\Gamma(s)} \int_{0}^{\varepsilon} t^{s-1} \mu_{n}(t) d t$ can be extended to a holomorphic function on $\left\{s \in C \mid \operatorname{Re}(s)>\frac{m+1}{2}-n-\frac{1}{2}\right\}$. Hence, by the usual argument, we get (1). (2) is due to the Tauberian theorem.

Our purpose was thus accomplished in such a way, but the meanings of the coefficients of the right hand side of (5.88) still remain obscure. Of course, we cannot expect them to be made perfectly clear. However, the constants $(5.80)$ can be made clearer through the independent study of $\operatorname{Tr}_{N} Z(t ; i)$, without using the formula (5.78). (In the former arguments, we have got the asymptotic expansion (5.79) not by using the concrete expressions (5.75) - (5.77) but by establishing the relation between $E_{C}$ and $E_{M}$.) If it is possible, conversely through the relation (5.80), we can get new informations on ${ }_{i}^{r} \tilde{a}_{j / 2},{ }_{2} A_{j / 2},{ }_{2} A$, etc. Therefore, it will be worth while to explore the possibility of its independent study.

The next section is devoted to the exploration of such a possibility.

\section{§ 6. Asymptotic behavior of $Z(; i)$.}

From Lemma 5.5, we know that

$$
\begin{aligned}
& \operatorname{Tr}_{N} Z(t ; d \tilde{c e}(i-1)+d r \wedge \tilde{c e}(i-1)) \\
& \quad=-2(2 t+1) \operatorname{Tr}_{N} Z(t ; \tilde{c e}(i-1))-4 t^{2} \frac{d}{d t} \operatorname{Tr}_{.} Z(t ; \tilde{c e}(i-1)),
\end{aligned}
$$




$$
\operatorname{Tr}_{N} Z(t ; d r \wedge \tilde{e}(i-1))=\operatorname{Tr}_{N} Z(t ; a d b c, \tilde{c e}(m+1-i)) .
$$

Now we make it the high aim to calculate the coefficients of the asymptotic expansion of $\operatorname{Tr}_{N} Z(t ; i)$, or thus equivalently, of $\operatorname{Tr}_{N} Z(t ; \tilde{c c}(i))$, as spectral invariants of $N$. However, our aim in this section is lower. Actually we restrict our attention only to the case where $i=0$.

Before stating our aim definitely, it may be better to make some preparations, first.

We denote points of $N$ simply by $x, y$, etc., i. e. delete " $~ "$.

Referring to Lemma 5.5, we can easily understand that

$$
\begin{aligned}
& \operatorname{Tr}_{N} Z(t ; 0)=\operatorname{Tr}_{N} Z(t ; \widetilde{c c}(0))=\int_{N} \operatorname{tr} Z(t, x) \widetilde{*} 1_{x}, \\
& \operatorname{tr} Z(t, x)=\frac{1}{2 t} e^{-1 / 2 t} \sum \sum_{j} I_{\nu_{j}}\left(\frac{1}{2 t}\right) \omega_{j}(x) \omega_{j}(x),
\end{aligned}
$$

where $\nu_{\jmath}=\sqrt{\mu_{j}+\alpha^{2}}, \quad \alpha=\alpha(0)=\frac{1-m}{2}, \quad \mu_{j}=\mu_{\tilde{c}(0), \jmath}$ and $\omega_{j}=\omega_{\tilde{c}(0), \jmath}$ Obviously, $\operatorname{tr} Z(t, x)$ is just the pointwise trace of

$$
\begin{aligned}
Z(t)=Z(t, x, y) & =\frac{1}{2 t} e^{-1 / 2 t} \sum_{\jmath} I_{\nu j}\left(\frac{1}{2 t}\right) \omega_{j}(x) \otimes \omega_{\jmath}(y) \\
& =\frac{1}{2 t} e^{-1 / 2 t} I_{\nu}\left(\frac{1}{2 t}\right),
\end{aligned}
$$

namely, that function of the operator $\nu=\sqrt{\tilde{\Delta}+\alpha^{2}}$ on $N$ which is parametrized by $t$.

By the way, it is relevant for us to rewrite (6.5) as follows; see (5.29).

$$
\begin{aligned}
Z(t)= & \frac{1}{2 \pi t} \sum_{j}\left\{\int_{0}^{\pi} e^{-(1-\cos s) / 2 t} \cos \nu_{j} s d s\right. \\
& \left.\quad-\sin \nu_{j} \pi \int_{0}^{\infty} e^{-(1+\cosh s) / 2 t} e^{-\nu_{j} s} d s\right\} \omega_{j}(x) \otimes \omega_{j}(y) \\
= & \frac{1}{2 \pi t}\left\{\int_{0}^{\pi} e^{-(1-\cos s) / 2 t} \cos \nu s d s-\sin \nu \pi \int_{0}^{\infty} e^{-(1+\cosh s) / 2 t} e^{-\nu s} d s\right\} .
\end{aligned}
$$

Let $\varphi(s)$ be a smooth function on $\boldsymbol{R}$ satisfying that $\varphi(s)=1$ near $s=0$ and $\varphi(s)=0$ for $|s|$, large, and let us decompose (6.6) as follows.

$$
\begin{aligned}
Z(t)= & \frac{1}{2 \pi t} \int_{0}^{\pi} \varphi(s) e^{-(1-\cos s) / 2 t} \cos \nu s d s \\
+ & \frac{1}{2 \pi t}\left\{\int_{0}^{\pi}(1-\varphi(s)) e^{-(1-\cos s) / 2 t} \cos \nu s d s\right. \\
& \left.-\sin \nu \pi \int_{0}^{\infty} e^{-(1+\cosh s) / 2 t} e^{-\nu s} d s\right\} .
\end{aligned}
$$


Then, since we have, for $\nu_{j}>0$,

$$
\begin{gathered}
\int_{0}^{\pi}(1-\varphi(s)) e^{-(1-\cos s) / 2 t} \cos \nu_{j} s d s-\sin \nu_{j} \pi \int_{0}^{\infty} e^{-(1+\cosh s) / 2 t} e^{-\nu_{j} s} d s \\
=(-1)^{n} \int_{0}^{\pi}\left(\frac{\partial}{\partial s}\right)^{2 n-1}\left((1-\varphi(s)) e^{-(1-\cos s) / 2 t}\right) \frac{\sin \nu_{j} s}{\nu_{j}^{2 n-1}} d s \\
\quad-\sin \nu_{j} \pi \int_{0}^{\infty}\left(\frac{\partial}{\partial s}\right)^{2 n-1}\left(e^{-(1+\cosh s) / 2 t)} \frac{e^{-\nu_{j} s}}{\nu_{j}^{2 n-1}} d s\right. \\
=(-1)^{n} \int_{0}^{\pi}\left(\frac{\partial}{\partial s}\right)^{2 n}\left((1-\varphi(s)) e^{-(1-\cos s) / 2 t)} \frac{\cos \nu_{j} s}{\nu_{j}^{2 n}} d s\right. \\
\quad-\sin \nu_{j} \pi \int_{0}^{\infty}\left(\frac{\partial}{\partial s}\right)^{2 n}\left(e^{-(1+\cosh s) / 2 t)} \frac{e^{-\nu_{j} s}}{\nu_{j}^{2 n}} d s,\right.
\end{gathered}
$$

the second term of the right hand side of (6.7) can be regarded as a smooth function on $N$ and, moreover, decreases exponentially when $t \downarrow 0$ because of the existence of the cutting-off function $\varphi$. Hence, for investigating the asymptotic expansion of (6.3) or (6.4), it suffices to set, in disregard of the second term of (6.7),

$$
\begin{aligned}
Z(t) & =\frac{1}{2 \pi t} \int_{0}^{\pi} \varphi(s) e^{-(1-\cos s) / 2 t} \cos \nu s d s \\
& =\frac{1}{4 \pi t} \int_{-\infty}^{\infty} \varphi(s) e^{-(1-\cos s) / 2 t} e^{-\imath s \nu} d s, \quad \imath=\sqrt{ }-1,
\end{aligned}
$$

and study that asymptotic expansion of its pointwise trace at $x$ which is equal to the asymptotic expansion of (6.4).

We can now state that our aim in this section is to explain the general algorithm which describes how to compute the coefficients of its pointwise asymptotic expansion at $x \in \operatorname{Int} N$ in terms of the informations which are derived from the behavior of the wave kernel $\cos \nu s$, or equivalently, the wave-like kernel $e^{-\imath s \nu}$, near the time $s=0$. More explicitly, since we have already known from (5.79) that, if $x \in \operatorname{Int} N$, then the pointwise trace $\operatorname{tr} Z(t, x)$ can be expanded into

$$
\operatorname{tr} Z(t, x) \sim \sum_{j=0}^{\infty} \tilde{\alpha}_{j}(x) t^{-(m+1) / 2+\jmath}, \quad t \downarrow 0,
$$

our aim is to explain how to compute the $\tilde{\alpha}_{j}$ 's in terms of the informations about the phase function and the amplitude of $e^{-2 s \nu}$.

Many of the results of this section can extend to the case where the objects are forms. But the expressions will become more complicated. This is the main reason why we restrict ourselves to the case where the objects are functions. However, the reason for setting $x \in \operatorname{Int} N$ is that, if $x \in \partial N$, then there seems to exist an essential difficulty: we will need more elaborate arguments in order to surmount such a difficulty. 
The remainder of this section is divided into three subsections as follows:

6.1. Review of the wave-like kernel on $N$

6.2. Key lemma

6.3. Computation of $\tilde{\alpha}_{\jmath}$

Please pay attention that we will use $D_{s}, \lambda_{-\jmath}, a_{-\jmath}, a_{k, l}^{\langle 2 n\rangle}, A_{-\jmath}$, etc. which resemble what were used in the former sections, in the senses which will be specified in the following.

\subsection{Review of the wave-like kernel on $N$.}

To study (6.9), we need to acquire the informations about the local behavior of the wave-like kernel $e^{-\imath s 2}\left(y_{1}, y_{2}\right)$ at $\left(s, y_{1}, y_{2}\right)=(0, x, x)$. In this subsection, on the basis of the Fourier integral operator theory, we will derive its property when the time $s$ is sufficiently close to 0 and $y_{1}, y_{2}$ belong to a sufficiently small neighborhood of $x \in \operatorname{Int} N$.

Let $\left(\boldsymbol{R}^{m},\left(x_{1}, \cdots, x_{m}\right)\right)$ be a local coordinate at $x \in \ln N$. Our Laplace (-Beltrami) operator has the following local expression on it.

$$
\begin{aligned}
\tilde{J} & =\frac{1}{\sqrt{\tilde{g}}(x)} \sum_{h, j} D_{x_{h}}\left(\tilde{g}^{h \jmath}(x) \sqrt{\tilde{g}(x)} D_{x_{j}}\right) \\
& =\sum_{h, j} \tilde{g}^{h j}(x) D_{x_{h}} D_{x_{j}}+\sqrt{-1} \sum_{h, j, k} \tilde{\Gamma}_{h j}^{k}(x) D_{x_{k}},
\end{aligned}
$$

where $\tilde{g}(x)=\operatorname{det}\left(\hat{g}_{h, j}(x)\right), D_{x_{h}}=\frac{1}{\sqrt{ }-1} \cdot \frac{\partial}{\partial x_{n}}$ and the $\tilde{\Gamma}_{h j}^{k}(x)$ 's are the Christoffel's symbols. Hence the symbol of the differential operator $\tilde{d}+\alpha^{2}$ is as follows.

$$
\sigma\left(\tilde{j}-\alpha^{2}\right)(x ; \xi)=\sum_{h, 3} \tilde{g}^{h}(x) \xi_{h} \xi,+\sqrt{-1} \sum_{h, j, k} \tilde{\Gamma}_{h j}^{k}(x) \xi_{k}+\alpha^{2} .
$$

The symbol of the positive square root $\nu=\sqrt{\tilde{\Delta}+\alpha^{2}}$, that is, a pseudo-differential operator, also admits an asymptotic expansion

$$
\sigma(\nu)(x ; \xi) \sim \sum_{j \geq-1} \lambda_{-j}(x ; \xi),
$$

with $\lambda_{-}$, homogeneous of degree $--\jmath$ with respect to $\xi$. By an easy computation, we have

$$
\begin{aligned}
& \lambda_{1}(x ; \xi)=p(x ; \xi)=\sqrt{ } \sum_{h, j} \tilde{g}^{h j}(x) \hat{\xi}_{h} \xi, \\
& \lambda_{u}(x ; \xi)=\sqrt{ }-1\left(2 \lambda_{1}(x ; \xi)\right)^{-1} \sum_{h, j, k} \tilde{I}_{h j}^{k}(x) \hat{\xi}_{k} .
\end{aligned}
$$

In general, according to [18] Page 298, we obtain

$$
R_{-\jmath}=\left(\lambda_{1}(x ; D)+\lambda_{0}(x ; D)+\cdots+\lambda_{-j}(x ; D)\right)-\left(\tilde{\boldsymbol{J}}+\alpha^{2}\right) \in O S P^{-\jmath}
$$

with $\lambda_{-j}(x ; \xi)=-\left(2 \lambda_{1}(x ; \xi)\right)^{-1} \sigma_{P}\left(R_{1-j}\right)$. Here $\sigma_{P}\left(R_{1-j}\right)$ is the principal symbol of $R_{1-\jmath}$. Hence we have 


$$
\sigma_{P}\left(R_{-j}\right)=\lambda_{0} \lambda_{-j}+\lambda_{-1} \lambda_{-j+1}+\cdots+\lambda_{-j} \lambda_{0},
$$

which combined with (6.14) implies

LEMMA 6.1. $\lambda_{-}(x ; \xi)$ is real, resp., imaginary if $\jmath$ is elen, resp., odd.

Now, setting $\lambda_{-j}(y ; \eta)=0$ for $|x-y|$, large, we will regard $\nu$ as a pseudodifferential operator on $\boldsymbol{R}^{m}$ and investigate the structure of the fundamental solution $e^{-\imath s \nu}$ of the initial-value problem of the first order hyperbolic equation;

$$
\left(D_{s}+\nu\right) u=0
$$

$$
u_{\mathrm{i} s=0}=u_{0} \text {. }
$$

According to Hörmander [13] or Duistermaat and Guillemin [8] (our notations imitate mainly those of $[8]), e^{-\imath s \nu}$ is a Fourier integral operator of class $I^{-1 / 4}$ with wave front set on the Lagrangian submanifold

$$
\begin{aligned}
A=\left\{((s ; \sigma),(y ; \eta),(x ; \xi)) \in T^{*}\left(\boldsymbol{R} \times N \times N^{*}\right) \backslash\{0\} ;\right. \\
\left.\sigma+p(y ; \eta)=0,(y ; \eta)=\Phi^{s}(x ;-\xi)\right\},
\end{aligned}
$$

where $\Phi^{s}$ denotes the $H_{p}$-flow in $T^{*} N \backslash\{0\}$, namely, the Hamiltonian flow defined by the principal symbol $p(x ; \xi)$ of $\nu$. We can find a phase function for $A$ near the set $\{(0, x,(x ; \xi))\}$ defined by

$$
\tilde{\phi}(s, y,(x ; \xi))=\left\langle\xi, \exp _{x}^{-1}(y)\right\rangle-s p(x ; \xi),
$$

where $\exp _{x}: T_{x} N \rightarrow N$ is the exponential map associated with the Riemannian metric ([12] Page 375). More generally, if we set, included a degree of freedom,

$$
\phi(y,(x ; \xi))=\left\langle\xi, \exp _{x}^{-1}(y)\right\rangle+O\left(\mid \exp _{x}^{-1}(y) !^{2}\right)
$$

which is homogeneous of degree 1 in $\xi$, then

$$
\phi(s, y,(x ; \xi))=\phi(y,(x ; \xi))-s p(x ; \xi)
$$

also defines a local phase function for $A$.

Now $e^{-\imath s \nu}$ can be written in the form, when $|s|$ is small,

$$
\begin{aligned}
& e^{-2 s \nu} \equiv \frac{1}{(2 \pi)^{m}} \int e^{\imath \dot{\rho}(s, y,(x ; \xi))} a(s, y,(x ; \xi)) d \xi \\
&= \frac{1}{(2 \pi)^{m}} \int_{s_{x}^{*} N} \int_{0}^{\infty} e^{\imath r(\dot{\xi}(y,(x ;()))-s)} \chi_{j}(r) \\
& \cdot a_{-j}(s, y,(x ; \omega)) r^{m-1-1} d r d \omega,
\end{aligned}
$$

where $r=p(x ; \xi), \quad a=\sum_{j \leq 0} \chi_{j} a_{-j} \in S^{0}\left(\boldsymbol{R} \times N \times\left(T^{*} N \backslash\{0\}\right)\right)$ with $a_{-j}$ homogeneous of degree $-j$ in $\xi$. Here " $\equiv$ " means "equivalent to each other modulo $C^{\infty}$-operators" and the $\chi$,'s are defined as follows. Let $\chi(r)$ be a smooth even function on $R$ 
which satisfies $\chi(r)=0$ for $r \in[0,1]$ and $\chi(r)=1$ for $r \in[2, \infty)$. Let $\left\{M_{j}\right\}$ be a sequence which goes to infinity very fast when $\jmath \uparrow \infty$. Then we set

$$
\chi_{j}(r)=\chi\left(\frac{r}{M_{j}}\right)
$$

Since our object, the $\tilde{\alpha}_{j}$ 's, are influenced by $C^{\infty}$-operators, a subtle argument will be needed for managing their influences. The above explanation how to construct the $\chi_{j}$ 's is a preparation for the argument.

We will collect the informations about the $a_{-}$'s from the eikonal equation and the transport equations. By applying $D_{s}+\nu_{y}$ formally to the right hand side of $(6.22)$, we get

$$
\frac{1}{(2 \pi)^{m}} \int e^{x \xi(s, y,(x ; \xi))} c(s, y,(x ; \xi)) d \xi,
$$

where

$$
\begin{gathered}
c(s, y,(x ; \xi))=-p(x ; \xi) a+\frac{1}{\sqrt{-1}} \cdot \frac{\partial a}{\partial s}+b, \\
b=e^{-\imath \sigma^{\prime}}\left(y ; D_{y}\right)\left(a e^{2 \phi}\right) \\
\sim \sum_{a} \frac{1}{\alpha !}\left(\partial_{\eta}^{(\alpha)} \lambda\right)\left(y ; \nabla_{y} \phi\right)\left[D_{y^{\prime}}^{(\alpha)}\left(a\left(s, y^{\prime},(x ; \xi)\right) e^{i h\left(y, y^{\prime},(x ; \xi)\right)}\right]_{\left(y^{\prime}=y\right.},\right. \\
h\left(y, v^{\prime},(x ; \xi)\right)=\phi\left(y^{\prime},(x ; \xi)\right)-\phi(y,(x ; \xi))-\left(y-y^{\prime}\right) \cdot \nabla_{x} \phi(y,(x ; \xi)) .
\end{gathered}
$$

Here $\partial_{\eta_{j}}=\frac{\partial}{\partial \eta_{j}}$ and $\boldsymbol{V}_{y} \phi=\left(\frac{\partial \phi}{\partial y_{1}}, \cdots, \frac{\partial \phi}{\partial y_{n}}\right)$. (6.25) admits an asymptotic expansion

$$
c(s, y,(x ; \xi)) \sim \sum_{j \geq-1} c_{-j}(s, y,(x ; \xi))
$$

with $c_{-\jmath}$ homogeneous of degree $-\jmath$ in $\xi$. By setting $c_{1}=0$, we get the eikonal equation

$$
p\left(y ; \nabla_{y} \phi\right)=p(x ; \xi),
$$

which determines $\phi$. Moreover, by setting $c_{0}=0$, we get the first transport equation

$$
\left(\frac{\partial}{\partial s}+Y\right) a_{0}(s, y,(x ; \xi))=0,
$$

where

$$
\begin{aligned}
Y=\sum_{\partial} \frac{\partial \lambda_{1}}{\partial \eta_{j}}\left(y ; \nabla_{y} \phi\right) \frac{\partial}{\partial y_{j}}+\sqrt{-1} \lambda_{0}\left(y ; \nabla_{y} \phi\right) \\
+\frac{1}{2} \sum_{j, k} \frac{\partial^{2} \lambda_{1}}{\partial \eta_{j} \partial \eta_{k}}\left(y ; \nabla_{y} \phi\right) \frac{\partial^{2} \phi}{\partial y_{j} \partial y_{k}} .
\end{aligned}
$$

In general, by setting $c_{-\jmath}=0, j=1,2, \cdots$, we get the transport equations 


$$
\left(\frac{\partial}{\partial s}+Y\right) a_{-j}+d_{-j}=0, \quad j=1,2, \cdots,
$$

where $d_{-}$, is expressible in terms of $\phi, a_{0}, a_{-1}, \cdots, a_{1-\jmath}$. The initial condition concerning $a_{0}$ is determined by

$$
a_{0}(0, x,(x ; \omega))=1 .
$$

In general, we set

$$
a_{-\jmath}(0, y,(x ; \omega))=0, \quad \jmath=1,2, \cdots .
$$

A function $f$ on $S_{x}^{*} N$ will be called an even, resp., odd function if $f(x ;-\omega)$ $=f(x ; \omega)$, resp., $f(x ;-\omega)=-f(x ; \omega)$. Then, referring to the proof of [8] Proposition 2.1 , we know

LEMMA 6.2. $\left(\frac{\partial}{\partial s}\right)^{k} a_{-j}(0, x,(x ; \omega))$ is an even, resp., odd function on $S^{*} N$ if $k-j$ is even, resp., odd.

By considering Lemma 6.1, we have

LEMMA 6.3. $a_{-\jmath}$ is real, resp., pure imaginary if $\mathrm{J}$ is even, resp., odd.

Proof. Since the initial condition of $a_{0}$ is real and the operator $Y$ is a real operator because of Lemma $6.1, a_{0}$ is real. Now, assume that the lemma is valid if $j<n$. If we can show that $d_{-n}$ is real, resp. pure imaginary if $n$ is even, resp. odd, then the lemma for $a_{-n}$ which is the solution of $(6.32)_{n}$ with the initial condition $(6.34)_{n}$ is obviously valid. Therefore, it suffices to prove that the above assertion for $d_{-n}$ is valid. Observing (6.26) and (6.27), we know that $d_{-n}$ is the sum of the following terms multiplied by real numbers:

$$
\frac{1}{i^{|\alpha|}} \partial_{\eta}^{\langle\alpha\rangle} \lambda_{-l} \partial_{y}^{\langle\beta\rangle} a_{-j}\left[\partial_{y^{\prime}}^{(\gamma)} e^{i h\left\langle y, y^{\prime},\langle x ; \xi)\right.}\right]_{i y^{\prime}=y}^{\langle n\rangle},
$$

where $j<n, \alpha=\beta+\gamma,-n+l+j+|\alpha| \geqq 0$ and $\left[\partial_{y^{\prime}}^{(\gamma)} e^{i n}\right]_{i y^{\prime}=y}^{\langle n\rangle}$ is the sum of those terms of $\left[\partial_{y^{\prime}}^{(\gamma)} e^{i h}\right]_{\mid y^{\prime}=y}$ whose degrees in $\xi$ equal to $-n+l+j+|\alpha|$. The degree of $\partial_{\eta}^{(\alpha)} \lambda_{-l} \partial_{y}^{(\beta)} a_{-}$, equals to $-l-j-|\alpha|$. Therefore, the assumption of the induction and Lemma 6.1 imply that $(6.35)$ is real, resp. pure imaginary if

$$
i^{-|\alpha|+(l+1)+(j+1)+\{-n+l+j+|\alpha|)}= \pm i^{-n}
$$

is real, resp. pure imaginary. Here we note that (6.36) is obtained from (6.35) by replacing $\partial_{\eta}^{(\alpha)} \lambda_{-l}, \partial_{y}^{(\beta)} a_{-\jmath}$, and $\left[\partial_{y}^{(\gamma)} e^{i n}\right]_{\mid y^{\prime}=y}^{\langle n\rangle}$ by $i^{l+1}, i^{j+1}$ and $i^{-n+l+\jmath+|\alpha|}$, respectively. This completes the proof.

Moreover, let $\phi^{+}(s, y,(x ; \xi))$ and $a^{+}(s, y,(x ; \xi)) \sim \sum_{j} a_{-j}^{+}$be the phase function and the amplitude of $e^{2 s \nu}$. Then, by the argument parallel to that of $e^{-2 s \nu}$, we get

$$
\phi^{+}(s, y,(x ; \xi))=\phi(y,(x ; \xi))+s p(x ; \xi)
$$




$$
a_{-j}^{+}(s)=a_{-j}(-s),
$$

where $\phi$ and $a_{-,}$are those of $e^{-2 \varepsilon \nu}$. Therefore,

$$
\begin{aligned}
2 \cos s \nu(y, x) & =e^{2 s \nu}+e^{-\imath s \nu} \\
\equiv & \frac{1}{(2 \pi)^{m}} \sum \iint e^{2 r \phi} \chi_{j}(r)\left\{\cos r s\left(a_{-j}(s)+a_{-j}(-s)\right)\right. \\
& \left.\quad+i \sin r s\left(a_{-j}(s)-a_{-j}(-s)\right)\right\} r^{m-1-\jmath} d r d \omega .
\end{aligned}
$$

Thus (6.39) is a real operator. Hence, by setting $y=x$, we know that

$$
\begin{aligned}
& \cos r s\left(a_{-j}(s, x,(x ; \omega))+a_{-j}(-s, x,(x ; \omega))\right) \\
& \quad+i \sin r s\left(a_{-j}(s, x,(x ; \omega))-a_{-j}(-s, x,(x ; \omega))\right)
\end{aligned}
$$

is a real function. Therefore, observing Lemma 6.3 , we get, for $|s|$, sufficiently small,

LEMMA 6.4. $a_{-\jmath}(s, x,(x ; \omega))$ is an even, resp., odd function of $s$ if $j$ is even, resp., odd.

\subsection{Key lemma.}

Let $u, v$ be arbitrarily fixed non-negative integers and $h(s)$ be a function on $R$ of class $C^{k+1}$ with compact support near $s=0$. Then our purpose of this subsection is to show that

$$
\int_{0}^{\pi} e^{-(1-\cos s) / 2 t}\left(\frac{\sin s}{2 t}\right)^{u}\left(\frac{\cos s}{2 t}\right)^{v} h(s) d s
$$

admits an asymptotic expansion when $t \downarrow 0$.

If we decompose (6.40) into $\int_{0}^{\pi / 2}+\int_{\pi / 2}^{\pi}$, then the term $\int_{\pi / 2}^{\pi}$ obviously decreases exponentially when $t \downarrow 0$. Hence, it suffices to study the asymptotic expansion of the term $\int_{0}^{\pi / 2}$. By the change of variables, $z=\frac{1-\cos s}{2 t}$, the term $\int_{0}^{\pi / 2}$ equals to

$$
\begin{aligned}
\frac{1}{2^{v}} t^{1 / 2-(u / 2+v)} \int_{0}^{1 / 2 t} e^{-z} z^{(u-1) / 2}(1-t z)^{(u-1) / 2} & \\
& \cdot(1-2 t z)^{v} h(\arccos (1-2 t z)) d z .
\end{aligned}
$$

Let us set

$$
f(w)=\left(1-w^{2}\right)^{(u-1) / 2}\left(1-2 w^{2}\right)^{v} h\left(\arccos \left(1-2 w^{2}\right)\right)
$$

and try to expand it when $w \downarrow 0$.

The term $\arccos \left(1-2 w^{2}\right)=2 \arccos |w|$ is an analytic function on $[0, \varepsilon)$ and admits the Taylor expansion as follows.

$$
\arccos \left(1-2 w^{2}\right)=\sum_{n=0}^{\infty} \frac{2((2 n-1) ! !)^{2}}{(2 n+1) !} w^{2 n+1}, \quad w \geqq 0,
$$


where $(2 n-1) ! !=(2 n-1)(2 n-3) \cdots 3 \cdot 1$ and $(-1) ! !=1$. On the other hand, we have the asymptotic expansion

$$
h(s) \sim \sum_{n=0}^{k} \frac{h^{(n)}(0)}{n !} s^{n}+O\left(s^{k+1}\right), \quad s \downarrow 0 .
$$

Since the first term of (6.43) is $2 w$, that is, not a constant term, we can get the following asymptotic expansion by substituting (6.43) for $s$ of (6.44).

$$
h\left(\arccos \left(1-2 w^{2}\right)\right) \sim \sum_{n=0}^{k} H_{k} w^{n}+O\left(w^{k+1}\right), \quad w \downarrow 0,
$$

where

$$
H_{0}=h(0), \quad H_{n}=\frac{2^{n}}{n !} h^{(n)}(0)+\sum_{l \geq 1} \widetilde{H}_{l} h^{(n-2 l)}(0)
$$

with $\tilde{H}_{l}$, a constant number. By the way, we have

$$
\begin{gathered}
\left(1-w^{2}\right)^{\langle u-1\rangle / 2}\left(1-2 w^{2}\right)^{v}=\sum_{n=0}^{\infty} B_{n}^{\langle u, v\rangle} w^{2 n}, \\
B_{n}^{\langle u, v\rangle=} \sum_{p+q=n} \frac{2^{q}}{p ! q !}\left(\frac{1-u}{2}\right)_{p}(-v)_{q}, \quad B_{n}^{\langle 0,0\rangle}=\frac{1}{2^{n}} \cdot \frac{(2 n-1) ! !}{n !},
\end{gathered}
$$

where $(a)_{n}=a(a+1) \cdots(a+n-1)$ and $(a)_{0}=1$. Hence, $f(w)$ admits the following asymptotic expansion.

$$
\begin{gathered}
f(w) \sim \sum_{n=0}^{k} F_{n}^{\langle u, v\rangle} w^{n}+O\left(w^{k+1}\right), \quad w \downarrow 0, \\
F_{n}^{\langle u, v\rangle}=\sum_{p+2 q=n} H_{p} B_{q}^{\langle u, v\rangle}, \quad F_{0}^{\langle u, v\rangle}=H_{0}=h(0) .
\end{gathered}
$$

Thus we get

$$
f\left((t z)^{1 / 2}\right) \sim \sum_{n=0}^{k} F_{n l}^{\langle u \cdot r\rangle} z^{n / 2} t^{n / 2}+O\left((t z)^{(k+1) / 2}\right), \quad t \downarrow 0 .
$$

Therefore, observing that

$$
\int_{0}^{1 / 2 t} e^{-z} z^{(u-1) / 2} z^{n / 2} d z=\int_{0}^{1 / 2 t} e^{-2} z^{(u+n+1) / 2-1} d z \rightarrow \Gamma\left(\frac{u+n+1}{2}\right), \quad t \downarrow 0,
$$

we can prove the following lemma by an easy induction.

KeY LemMa 6.5.

$$
\begin{aligned}
& \int_{0}^{\pi} e^{-(1-\cos s) / 2 t}\left(\frac{\sin s}{2 t}\right)^{u}\left(\frac{\cos s}{2 t}\right)^{v} h(s) d s \\
& \sim \sum_{n=0}^{k} 2^{-v} F_{n}^{\langle u, v\rangle} \Gamma\left(\frac{u+n+1}{2}\right) t^{(n+1) / 2-(u / 2+v)}+O\left(t^{(k+2) / 2-(u / 2+v)}\right.
\end{aligned}
$$


In many cases, we use (6.51) by assuming $k=\infty, u=v=0$ and $h(0)=h^{(1)}(0)$ $=\cdots=h^{\left(n_{0}-1\right)}(0)=0$ for some $n_{0}$. On the above assumption, (6.51) can be expanded as follows:

$$
\sum_{n=n_{0}}^{\infty} F_{n}^{\langle 0,0\rangle} \Gamma\left(\frac{n+1}{2}\right) t^{(n+1\rangle / 2}, \quad F_{n}^{\langle 0,0\rangle}=\frac{2^{n}}{n !} h^{(n)}(0) .
$$

Here (6.52) consists of the terms which involve the half-power of $t$, resp. the terms which consist of the integer-powers of $t$ if $h(s)$ is an even, resp. odd function.

\subsection{Computation of $\tilde{\alpha}_{j}$.}

Observing (6.4), (6.9) and (6.22), we get

$$
\begin{aligned}
\operatorname{tr} Z(t, x) \equiv \frac{1}{(2 \pi)^{m+1} 2 t} \iint_{-\infty}^{\infty} e^{2 \psi(s, x,(x ; \xi))} \\
=\frac{1}{(2 \pi)^{m+1} 2 t} \sum_{j \geqq 0} \int_{S_{x}^{*} N} \int_{0}^{\infty} \int_{-\infty}^{\infty} e^{-\imath s r} e^{-(1-\cos s) / 2 t} a(s, x,(x ; \xi)) d s d \xi \\
\cdot \chi_{j}(r) a_{-j}(s, x,(x ; \omega)) r^{m-1-\jmath} d s d r d \omega,
\end{aligned}
$$

where $a_{-j}(s)$ is an either even or odd function and $a_{-j}(s)=0$ for $|s|$, large.

The purpose of this subsection is to calculate the $\tilde{\alpha}_{j}$ 's with the help of the formula (6.53). However, to my regret, all I can show on the basis of the informations mentioned in Subsection 6.1 is the existence of the following asymptotic expansion (6.55) with (6.56) and the way of the computations of its coefficients. In this paper we will not give the full descriptions of the formulas of the coefficients because of their complexities: if necessary, sum up those terms of the various asymptotic expansions described in due course whose orders in $t$ equal to the one fixed arbitrarily.

$$
\operatorname{tr} Z(t, x) \sim \begin{cases}\sum_{j=0}^{\infty} \tilde{\alpha}_{j}(x) t^{-(m+1) / 2+\jmath}+\sum_{\substack{m \leq j<\infty \\ \jmath: \text { odd }}} \tilde{\beta}_{j / 2}(x) t^{-(m+1) / 2+j / 2} ; & m: \text { odd } \\ \sum_{j=0}^{\infty} \tilde{\alpha}_{j}(x) t^{-(m+1) / 2+\jmath}+\sum_{\jmath=m / 2+1} \tilde{\gamma}_{j}(x) t^{-(m+1) / 2+\jmath} \log t ; m: \text { even } & \\ \tilde{\alpha}_{0}(x)=(4 \pi)^{-(m+1) / 2} & \end{cases}
$$

As a direct consequence of $(6.10)$, we have

$$
\tilde{\beta}_{j / 2}(x)=\tilde{\gamma}_{j}(x)=0 \text {. }
$$

From our standpoint, this ought to be verified by the investigation of the wavelike kernel, independently of (6.10). However, to do so, closer investigations than those mentioned in Subsection 6.1 will be needed. Hence, we have omitted the approach from such a standpoint. 
When we use the formula (6.54) for the study, we ought to pay attention to the following fact.

If $K(s)$ is a smooth function on $\boldsymbol{R}$, then, from (6.52), we know that

$$
\begin{aligned}
\int_{-\infty}^{\infty} e^{-(1-\cos s) / 2 t} \varphi(s) K(s) d s & =\int_{0}^{\infty} e^{-(1-\cos s) / 2 t} \varphi(s)(K(s)+K(-s)) d s \\
& \sim \sum_{n=0} F_{n}^{\langle 0,0\rangle} \Gamma\left(\frac{n+1}{2}\right) t^{(n+1) / 2}, \quad t \downarrow 0,
\end{aligned}
$$

where the $F_{n}^{\langle 0,0\rangle}$ s are constant numbers, in particular, $F_{0}^{\langle 0,0\rangle}=2 K(0)$. Hence, much care is necessary in replacing an operator for the one which is equivalent to the former one modulo $C^{\infty}$-operators.

We will first investigate those terms of (6.54) with $j \leqq m-1$.

$$
\begin{aligned}
& \int_{0}^{\infty} \int_{-\infty}^{\infty} e^{-\imath s r} e^{-(1-\cos s) / 2 t} \chi_{j}(r) a_{-j}(s) r^{m-1-\jmath} d s d r \\
= & \int_{0}^{\infty} \chi_{j}(r) r^{m-1-\jmath}\left\{\int_{-\infty}^{\infty} e^{-\imath s r} e^{-(1-\cos s) / 2 t} a_{-\jmath}(s) d s\right\} d r \\
\equiv & \int_{0}^{\infty} r^{m-1-\jmath}\left\{\int_{-\infty}^{\infty} e^{-\imath s r} e^{-(1-\cos s) / 2 t} a_{-j}(s) d s\right\} d r \\
= & \int_{0}^{\infty}\left\{\int_{-\infty}^{\infty} e^{-\imath s \tau}\left(\frac{1}{i} \frac{\partial}{\partial s}\right)^{m-1-\jmath}\left(e^{-(1-\cos s) / 2 t} a_{-j}(s)\right) d s\right\} d r \\
= & \int_{-\infty}^{\infty} \mathscr{T}(Y)(s)\left(\frac{1}{2} \frac{\partial}{\partial s}\right)^{m-1-\jmath}\left(e^{-(1-\cos s) / 2 t} a_{-j}(s)\right) d s .
\end{aligned}
$$

Here $Y(s)$ is the Heaviside function and $\mathcal{F}(Y)$ is its Fourier transform (in the distribution sense), that is,

$$
\mathscr{I}(Y)(s)=\hat{Y}(s)=\int_{-\infty}^{\infty} e^{-\imath s r} Y(r) d r .
$$

Hence we have

$$
\mathscr{F}(Y)(s)=\pi \delta(s)+\frac{1}{\imath} v \cdot p \cdot \frac{1}{s}
$$

where $\delta(s)$ is the Dirac operator and $v \cdot p \cdot \frac{1}{s}$ is a distribution defined by the principal value of $\frac{1}{s}$ at $s=0$ ([14] II Page 274). Therefore, $(6.60)$ equals to

$$
\begin{aligned}
& \left(\frac{1}{i} \frac{\partial}{\partial s}\right)^{m-1-\jmath}\left[e^{-(1-\cos s) / 2 t} a_{-j}(s)\right]_{\mid s=0} \\
& \quad+\frac{1}{\imath} v \cdot p \cdot \int_{-\infty}^{\infty} \frac{1}{s}\left(\frac{1}{\imath} \frac{\partial}{\partial s}\right)^{m-1-\jmath}\left[e^{-(1-\cos s) / 2 t} a_{-j}(s)\right] d s .
\end{aligned}
$$

For studying the asymptotic expansion of (6.63), it suffices to differentiate 
$e^{-(1-\cos s) / 2 t} a_{-j}(s)$ with the help of the formula (5.31) and to apply Key lemma 6.5 to it. However, (5.31) is not a handy kind of formula. The following will be a little more convenient than (5.31) for our purpose.

LEMMA 6.6. Given $n \in \boldsymbol{N}$,

$$
\begin{aligned}
& \left(\frac{\partial}{\partial S}\right)^{2 n}\left(e^{-(1-\cos s) / 2 t}\right)=e^{-(1-\cos s\rangle / 2 t} \sum_{0 \leq k \leq l \leq n} a_{k, l}^{\langle 2 n\rangle}\left(\frac{\sin s}{2 t}\right)^{2 n-2 l}\left(\frac{\cos s}{2 t}\right)^{k}, \\
& \left(\frac{\partial}{\partial s}\right)^{2 n+1}\left(e^{-(1-\cos s) / 2 l}\right)=e^{-(1-\cos s) ; 2 t} \sum_{0 \leq k \leq l \leq n} b_{k, l}^{\langle 2 n+1)}\left(\frac{\sin s}{2 t}\right)^{2 n+1-2 l}\left(\frac{\cos s}{2 t}\right)^{k},
\end{aligned}
$$

where the coefficients have the follouing relations;

$$
a_{0,0}^{\langle 0\rangle}=1 \text {, }
$$

$$
\begin{aligned}
& a_{k, l}^{\langle 2 n\rangle}= \begin{cases}-b_{k, l}^{\langle 2 n+1\rangle}+(2 n+1-2 l) b_{k-1, l-1}^{\langle 2 n-1\rangle}-(k+1) b_{k+1, l}^{\langle 2 n-1\rangle} ; & 0 \leqq l \leqq n-1, \\
b_{k-1, n-1}^{\langle 2 n-1\rangle} & ; l=n,\end{cases} \\
& b_{k, l}^{\langle 2 n+1\rangle}=-a_{k, l}^{\langle 2 n\rangle}+(2 n+2-2 l) b_{k-1, l-1}^{\langle 2 n\rangle}-(k+1) a_{k+1, l}^{\langle 2 n\rangle} .
\end{aligned}
$$

Proof. The lemma can be verified by an easy induction.

LEMMA 6.7.

$$
a_{l, l}^{2 n\rangle}=(-1)^{l}\left(\begin{array}{l}
n \\
l
\end{array}\right) \frac{(2 n-1) ! !}{(2 n-2 l-1) ! !} .
$$

Proof. According to (6.66), the lemma can be verified easily by induction. Here, we will compute $a_{n, n}^{\langle 2 n\rangle}$, which provides the starting point of our inductive proof. From (6.63), we know

$$
\left(\frac{\partial}{\partial s}\right)^{2 n}\left(e^{-(1-\cos s) / 2 t}\right)_{i s=0}=a_{n, n}^{\langle 2 n\rangle}\left(\frac{1}{2 t}\right)^{n} .
$$

Hence, observing (5.31), $a_{n, n}^{\langle 2 n\rangle}$ equals to

$$
\sum_{k=0}^{n}-\frac{(-1)^{n-k}}{n !}\left(\begin{array}{l}
n \\
k
\end{array}\right)\left(\frac{\partial}{\partial s}\right)^{2 n}\left(\cos ^{k} s\right)_{s=0}==\sum_{k=0}^{n} \frac{(-1)^{k}}{n !}\left(\begin{array}{l}
n \\
k
\end{array}\right)_{\substack{p_{1}+\cdots+p_{k}=2 n \\
p_{j}: \text { even } \geq 0}} \frac{(2 n) !}{p_{1} ! \cdots p_{k} !} .
$$

Set $A_{l}=\sum_{\substack{p_{1}+\ldots+p_{l}=2 n \\ p_{j}: \text { even }>0}} \frac{1}{p_{1} ! \cdots p_{l} !}$ and rearrange the above so as to $\sum_{i=1}^{n} a_{l} A_{l}$. Then,

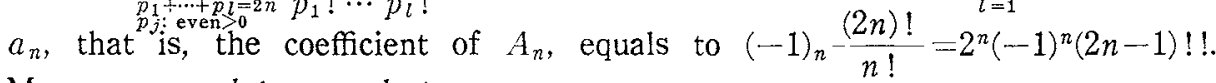
Moreover, $a_{l}, l<n$, equals to

$$
\frac{(2 n) !}{n !}(-1)^{n} \sum_{j=0}^{n-l}(-1)^{j}\left(\begin{array}{c}
n \\
n-j
\end{array}\right)\left(\begin{array}{c}
n-j \\
n-l-j
\end{array}\right)=(-1)^{n} \frac{(2 n) !}{n !}\left(\begin{array}{c}
n \\
l
\end{array}\right) \sum_{j=0}^{n-l}(-1)^{j}\left(\begin{array}{c}
n-l \\
j
\end{array}\right)=0 .
$$

Thus the proof of $a_{n, n}^{\langle 2 n\rangle}=(-1)^{n}(2 n-1) !$ ! is complete.

By using Lemma 6.6, we can show that the first and the second terms of 
(6.63), respectively, equal to

$$
\begin{aligned}
& \pi\left(\frac{1}{i}\right)^{m-1-\jmath} \sum_{\substack{2 p+q=m-1-j \\
0 \leq m \leq p}}\left(\begin{array}{c}
m-1-j \\
2 p
\end{array}\right) a_{k, i}^{\langle 2 p ;} a_{-j}(q)(0)(2 t)^{-k}, \\
& \left(\frac{1}{i}\right)^{m-3} \sum_{\substack{2 p+q=m-1-j \\
0 \leq k \leq l \leq p}}\left(\begin{array}{c}
m-1-\jmath \\
2 p
\end{array}\right) a_{k, i}^{\langle 2,\rangle}
\end{aligned}
$$

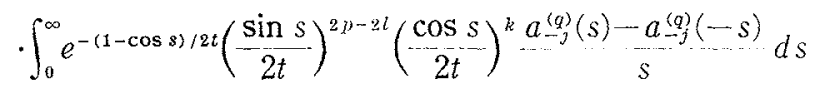

$$
\begin{aligned}
& +\left(\frac{1}{i}\right)^{m-j} \sum_{\substack{2 p+1+q=m-1-j \\
0 \leq k \leq l \leq p}}\left(\begin{array}{c}
m-1-j \\
2 p+1
\end{array}\right) \frac{b_{k, 2}^{2 p+1\rangle}}{2 t} \\
& \cdot \int_{0}^{\infty} e^{-(1-\cos s) / 2 t}\left(\frac{\sin s}{2 t}\right)^{2 p-2 l}\left(\frac{\cos s}{2 t}\right)^{k} \sin s\left(a_{--j}^{(q)}(s)+a_{-\jmath}^{(q)}(--s)\right) d s,
\end{aligned}
$$

where $a_{-\jmath}^{(q)}(s)=\left(\frac{\partial}{\partial s}\right)^{q} a_{-\jmath}(s)$.

(6.68) is a polynomial of $t^{-1}$. Hence, it suffices to show that (6.69) admits an asymptotic expansion. Set

$$
\begin{aligned}
& A_{-j}^{\langle q\rangle}(s)=\frac{a_{-j}^{(q)}(s)-a_{-j}^{(q)}(-s)}{s} \\
& B_{-j}^{\langle q\rangle}(s)=\frac{\sin s}{s}\left(a_{-j}^{(q)}(s)+a_{-j}^{(q)}(-s)\right) .
\end{aligned}
$$

These are smooth functions and satisfy

$$
\begin{aligned}
& \left(\frac{\partial}{\partial s}\right)^{n} A_{-j}^{\langle Q\rangle}(0)= \begin{cases}\left.\frac{2}{n+2} a_{-j}^{\langle q ;} n+1\right)(0) & \text { if } n \text { is even, } \\
0 & \text { if } n \text { is odd, }\end{cases} \\
& \left(\frac{\partial}{\partial s}\right)^{n} B_{-j}^{\langle Q\rangle}(0)= \begin{cases}\sum_{k=0}^{n / 2} \frac{2(-1)^{n / 2-k}}{n+1-2 k}\left(\begin{array}{c}
n \\
2 k
\end{array}\right) a_{-(q+3 k)}^{(0)} & \text { if } n \text { is even, } \\
0 & \text { if } n \text { is odd. }\end{cases}
\end{aligned}
$$

Referring to Key lemma 6.5, we set

$$
\begin{aligned}
& F\left(A_{-j}^{\langle q\rangle}\right)_{n}^{\langle u, v\rangle}=" F_{n}^{\langle u, v\rangle} \text { with } h(s)=A_{-j}^{\langle q\rangle}(s) ", \\
& F\left(B_{-j}^{\langle q\rangle}\right)_{n}^{\langle u, v\rangle}=" F_{n}^{\langle u, v\rangle} \text { with } h(s)=B_{--j}^{\langle q\rangle}(s) " .
\end{aligned}
$$

Then, since $F\left(A_{-j}^{\langle q\rangle}\right)_{n}^{\langle u, v\rangle}=F\left(B_{-j}^{\langle q}\right)_{n}^{\langle u, v\rangle}=0$ if $n$ is odd, (6.69) admits the following asymptotic expansion; see (6.45) and (6.49).

$$
\left(\frac{1}{\imath}\right)^{m-\jmath} \sum_{\substack{0 \leq n<\infty \\
n: \text { even }}} \sum_{\substack{p+q=m-1-j \\
0 \leqq k \leqq l \leqq p}}\left(\begin{array}{c}
m-1-j \\
2 p
\end{array}\right) a_{k, l}^{\{2 p>} \frac{\Gamma\left(\frac{n+1}{2}+p-l\right)}{2^{k}}
$$

$$
\cdot F\left(A_{-j}^{\langle q\rangle}\right)_{n}^{\langle 2 p-2 l, k\rangle} t^{(n+1) / 2-(p-z+k)}
$$




$$
\begin{aligned}
& +\left(\frac{1}{\imath}\right)^{m-\jmath} \sum_{\substack{0 \leq n<\infty \\
n: \text { even }}} \sum_{\substack{2 p+1+q=m-1-j \\
0 \leq k \leq l \leq p}}\left(\begin{array}{c}
m-1-\jmath \\
2 p+1
\end{array}\right) b_{k, l}^{\langle 2 p+1\rangle} \frac{\Gamma\left(\frac{n+1}{2}+p-l\right)}{2^{k+1}} \\
& \text { - } F\left(B_{-j}^{\langle q\rangle}\right)_{n}^{\langle 2 p-2 l, k\rangle} t^{(n-1) / 2-(p-l+k)} \text {. }
\end{aligned}
$$

In order to complete our task of investigating the terms with $\jmath \leqq m-1$, we have only to integrate (6.68) and (6.73) over $S_{x}^{*} N$. Though it seems that the resulting asymptotic expansions have imaginary parts, they are, of course, real. Actually, as for (6.68), if $m$ is odd, then $\left(\frac{1}{2}\right)^{m-1-3} a_{-j}^{(q)}(0)$ is real because of Lemma 6.3, and, if $m$ is even, then $\int_{S_{x}^{*} N} a^{(q)}(0) d \omega=0$ for any $j$ because of Lemma 6.2. Moreover, as for (6.73), the first, resp. the second sums of (6.73) are the power series of $t$ with the coefficients which equal to certain finite sums of the $\left(\frac{1}{\imath}\right)^{m-\jmath} a_{-\jmath}^{(q+\text { odd) }}(0)$ 's, resp. the $\left(\frac{1}{2}\right)^{m-\jmath} a_{-\jmath}^{(q+\text { even) }}(0)^{\prime}$ 's multiplied by real numbers; if $m$ is odd, then their integrations, that is, $\int_{S_{x}^{*} N}\left(\frac{1}{\imath}\right)^{m-j} a_{-j}^{(q+o d d)}(0) d \omega$ and $\int_{S_{x}^{*} N}\left(\frac{1}{\imath}\right)^{m-\jmath} a_{j}^{(q+\text { even })}(0) d \omega$, equal to 0 because of Lemma 6.2 , if $m$ is even, then they are real because of Lemma 6.3. Besides, from the other view-point, these argument verifies that, if $m$ is odd, then our asymptotic expansion is put under the control of (6.68) which consists of those terms whose orders in t are of integerpowers, and, if $m$ is even, then it is put under the control of (6.73) which consists of those terms whose orders in t are of half-integer-powers.

Thus we get the following results.

The expression

$$
\frac{1}{(2 \pi)^{m+1} 2 t} \sum_{m-1 \geq j} \int_{S_{x}^{*} N} \int_{0}^{\infty} \int_{-\infty}^{\infty} e^{-\imath s r} e^{-\langle 1-\cos s) / 2 t} a_{-j}(s) r^{m-1-\jmath} d s d r d \omega
$$

admits the following asymptotic expansion:

$$
\begin{array}{ll}
\sum_{j=0}^{(m-1) / 2} \tilde{\alpha}_{1, j}(x) t^{-(m+1) / 2+\jmath} & \text { if } m \text { is odd, } \\
\sum_{j=0}^{\infty} \tilde{\alpha}_{1, \jmath}(x) t^{-(m+1) / 2+\jmath} & \text { if } m \text { is even. }
\end{array}
$$

Besides, we get, by an easy computation,

$$
\tilde{\alpha}_{1,0}(x)=(4 \pi)^{-(m+1) / 2} \text {. }
$$

Actually, if $m$ is odd, then, in view of (6.68), (6.33) and (6.67), we have

$$
\begin{aligned}
\tilde{\alpha}_{1,0}(x) & =(2 \pi)^{-(m+1)} 2^{-1} \pi a_{(m-1) / 2,(m-1) / 2}^{\langle m-1\rangle}(-2)^{-(m-1) / 2} \operatorname{vol}\left(S_{x}^{*} N\right) \\
& =(4 \pi)^{-(m+1) / 2},
\end{aligned}
$$

and, if $m$ is even, then, in view of $(6.73),(6.33),(6.66)$ and $(6.67)$ we have, 


$$
\begin{aligned}
\tilde{a}_{1,0}(x) & =(2 \pi)^{-(m+1)} 2^{-1}(-1)^{m / 2} \sum_{0 \leqq l \leqq m / 2-1} b_{l, l}^{\langle m-1\rangle} \frac{\Gamma\left(\frac{m-1}{2}-l\right)}{2^{l}} \operatorname{vol}\left(S_{x}^{*} N\right) \\
& =\frac{(-1)^{m / 2}}{2^{(m-2) / 2}\left(\frac{m-2}{2}\right) !(4 \pi)^{(m+1) / 2}} \sum_{0 \leq l \leq m / 2-1}(m-3-2 l) ! ! b_{l, l}^{\langle m-1\rangle} \\
& =\frac{(-1)^{m / 2}(m-1) ! !}{2^{(m-2) / 2}\left(\frac{m-2}{2}\right) !(4 \pi)^{(m+1) / 2}} \sum_{0 \leqq l \leqq m / 2-1} \frac{(-1)^{l}}{l-(m-1) / 2}\left(\begin{array}{c}
(m-2) / 2 \\
l
\end{array}\right) \\
& =\frac{(-1)^{m / 2}}{2^{(m-2) / 2}\left(\frac{m-2}{2}\right) !(4 \pi)^{(m+1) / 2}}(-1)^{m / 2} 2^{m / 2-1}\left(\frac{m-2}{2}\right) ! \\
& =(4 \pi)^{-(m+1) / 2},
\end{aligned}
$$

where the last equality but one is due to [14] II Page 12 . Or, more easily, since $\tilde{\alpha}_{1,0}(x)$ is a value which can be computed by an elementary algebraic method (that is, $\tilde{\alpha}_{1,0}(x)$ does not depend on $x$ or $\left.N\right),(6.77)$ can be regarded as a direct consequence of the case where $N^{m}=S^{m}$, that is, $C(N)=\boldsymbol{R}^{m+1} \backslash\{0\}$.

Next, before investigating those terms of $(6.54)$ with $j>m-1$, we will deal with the influence of $C^{\infty}$-operators; see (6.58). Regarding the left hand side of (6.53) as the trace of the right hand side of (6.9), we set

$$
\begin{aligned}
\int_{-\infty}^{\infty} e^{-(1-\cos s) / 2 t} k_{1}(s, t, x) d s= & \text { "the l.h.s. of }(6.53) " \\
& \quad \text { - "the r.h.s. of }(6.53) ", \\
\int_{-\infty}^{\infty} e^{-(1-\cos s) / 2 t} k_{2}(s, t, x) d s= & \frac{1}{(2 \pi)^{m+1} 2 t} \int_{S_{x}^{*} N}\{“(6.59) "-“(6.60) "\} d \omega .
\end{aligned}
$$

Then $k(s)=k_{1}(s)+k_{2}(s)$ is a smooth function and, moreover, can be regarded as an even function. Hence, from (6.58), we have

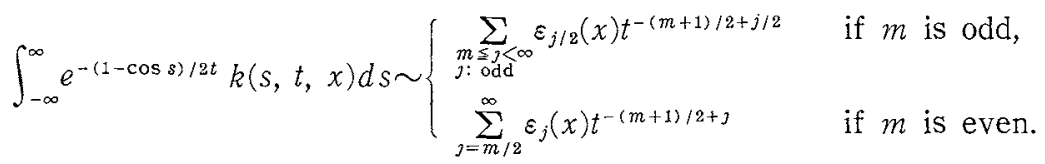

We will now deal with those terms of (6.54) with $\jmath>m-1$.

$$
\begin{aligned}
& \int_{0}^{\infty} \int_{-\infty}^{\infty} e^{-\imath s r} e^{-(1-\cos s) / 2 t} \chi_{j}(r) a_{-j}(s) r^{m-1-\jmath} d s d r \\
= & \int_{0}^{\infty} \chi_{j}(r) r^{-(j-m+1)}\left\{\int_{-\infty}^{\infty} e^{-\imath s r} e^{-(1-\cos s) / 2 t} a_{-\jmath}(s) d s\right\} d r .
\end{aligned}
$$

We will first rewrite (6.83) so that we can apply Key lemma 6.5 to it. (6.83) is 
a real function of $t$ and, moreover, $a_{-j}(s)$ has the property mentioned at Lemma 6.3. Hence, it suffices to deal with

$$
\operatorname{Re} \int_{0}^{\infty} \chi(r) r^{-k} \hat{f}(r) d r, \quad k \in N,
$$

in the four cases, namely,

Case (i): $k$ is even and $f(s)=g(s)$,

Case (ii): $k$ is even and $f(s)=i h(s)$,

Case (iii): $k$ is odd and $f(s)=g(s)$,

Case (iv): $k$ is odd and $f(s)=i h(s)$,

where $g(s)$ and $h(s)$ are real functions. Actually, by setting $\chi(r)=\chi_{j}(r), k=\jmath-m+1$ and $f(s)=e^{-(1-\cos s) / 2 t} a_{-j}(s),(6.83)$ can be classified into a certain one of the above cases.

Now, (6.84) equals to

$$
\begin{aligned}
& \operatorname{Re} \int_{-\infty}^{\infty} \mathscr{I}\left(\chi(r)|r|^{-k}+\chi(r)|r|^{-k} \operatorname{sgn} r\right) \frac{f(s)}{2} d s \\
= & \operatorname{Re} \int_{-\infty}^{\infty}\left\{\mathscr{F}\left(|r|^{-k}\right)(s\rangle+\mathscr{F}\left(|r|^{-k} \operatorname{sgn} r\right)(s)\right\} \frac{f(s)}{2} d s \\
& -\frac{1}{2 \pi} \operatorname{Re} \int_{-\infty}^{\infty}\left\{\hat{\phi} * \mathscr{F}\left(|r|^{-k}\right)+\hat{\psi} * \mathscr{F}\left(|r|^{-k} \operatorname{sgn} r\right)\right\} \frac{f(s)}{2} d s,
\end{aligned}
$$

where $\phi(r)=1-\chi(r)$. Moreover, from [14, II Page 274], etc.,

$$
I\left(|r|^{-k}\right)(s)= \begin{cases}\frac{(-1)^{k / 2} \pi}{\Gamma(k)}|s|^{k-1} & \text { if } k \text { is even, } \\ \frac{(-1)^{(k+1) / 2}}{\Gamma(k)}-\cdots s^{k-1}(2 \gamma+2 \log |s|) & \text { if } k \text { is odd, }\end{cases}
$$

$$
\mathscr{I}\left(|r|^{-k} \operatorname{sgn} r\right)(s)= \begin{cases}\frac{(-1)^{(k+2) / 2}}{\Gamma(k)}-\imath s^{k-1}(2 \gamma+2 \log |s|) & \text { if } k \text { is even, } \\ \left.\left.\frac{(-1)^{(k+1) / 2} \pi}{\Gamma(k)} i\right|_{\mid}\right|^{k-1} \operatorname{sgn} s & \text { if } k \text { is odd, }\end{cases}
$$

where $\gamma$ is the Euler's constant. Applying (6.86) and (6.87) to (6.85), we get the following results. Let's set

$$
G(s)=\frac{1}{2}(g(s)+g(-s)), \quad H(s)=\frac{1}{2}(h(s)-h(-s)) .
$$

In Case (i), (6.85) equals to

$$
\frac{(-1)^{k / 2} \pi}{\Gamma(k)} \int_{0}^{\infty} s^{k-1} G(s) d s-\frac{(-1)^{k / 2}}{2 \Gamma(k)} \int_{0}^{\infty}\left(\hat{\zeta} *|s|^{k-1}\right) G(s) d s .
$$

For the further rewriting of the second term of (6.88), the following formula is 
useful.

$$
\begin{aligned}
& \left(\hat{\psi} *|s|^{k-1}\right)(s)=\int_{-\infty}^{\infty}|s-t|^{k-1} \hat{\phi}(t) d t \\
& =\sum_{\substack{p+q=k-1 \\
p: \text { odd }}}\left(\begin{array}{c}
k-1 \\
p
\end{array}\right) s^{p} \int_{-s}^{s} \widehat{D_{r}^{q} \phi(t)} d t \\
& +\sum_{\substack{p+q=k-1 \\
p: \text { even }}}\left(\begin{array}{c}
k-1 \\
p
\end{array}\right) s^{p}\left\{\int_{s}^{\infty} \widehat{D_{r}^{q} \psi(t)} d t-\leftarrow \int_{-s}^{\infty} \widehat{D_{r}^{q} \psi(t)} d t\right\} .
\end{aligned}
$$

In Case (ii), (6.85), that is, the first term of the right hand side of (6.85), equals to

$$
\frac{(-1)^{k / 2} 2 \gamma}{\Gamma(k)}-\int_{0}^{\infty} s^{k-1} H(s) d s+\frac{(-1)^{k / 2} 2}{\Gamma(k)} \int_{0}^{\infty} s^{k-1} H(s) \log s d s,
$$

and, $(6.85)_{2}$, that is, the second term of the right hand side of (6.85), equals to

$$
\begin{aligned}
& -\frac{(-1)^{k / 2} 2 \gamma}{\Gamma(k)} \int_{0}^{\infty} s^{k-1} H(s) d s \\
& \quad-\frac{(-1)^{(k+1) / 2}}{\pi \Gamma(k)} \sum_{p+q=k-1}(-1)^{q}\left(\begin{array}{c}
k-1 \\
p
\end{array}\right) \int_{n}^{\infty}\left(\widehat{D} \widehat{r}_{r}^{q} \psi \log |s|\right) s^{n} H(s) d s .
\end{aligned}
$$

Here we have used the formula

$$
\begin{aligned}
& \hat{\psi} *\left(s^{k-1}(2 \gamma+2 \log |s|)\right) \\
& =4 \pi \gamma s^{k-1}+\sum_{p+q=k-1}(-1)^{q}\left(\begin{array}{c}
k-1 \\
p
\end{array}\right) s^{p}\left(\widehat{D_{r}^{q} \psi} * \log |s|\right) .
\end{aligned}
$$

In Case (iii), similarly to Case (ii), $(6.85)_{1}$ and $(6.85)_{2}$ equal to

$$
\begin{aligned}
& \frac{(-1)^{(k+1) / 2} 2 \gamma}{\Gamma(k)} \int_{0}^{\infty} s^{k-1} G(s) d s+\frac{(-1)^{(k+1) / 2} 2}{\Gamma(k)} \int_{0}^{\infty} s^{k-1} G(s) \log s d s, \\
& -\frac{(-1)^{(k+1) / 2} 2 \gamma}{\Gamma(k)} \int_{0}^{\infty} s^{k-1} G(s) d s \\
& -\frac{(-1)^{(k+1) / 2}}{\pi \Gamma(k)} \sum_{p+q=k-1}(-1)^{q}\left(\begin{array}{c}
k-1 \\
p
\end{array}\right) \int_{0}^{\infty}\left(D_{r}^{q} \phi * \log |s|\right) s^{p} G(s) d s .
\end{aligned}
$$

In Case (iv), similarly to Case (i), (6.85) equals to

$$
\begin{aligned}
& -\frac{(-1)^{(k+1) / 2} \pi}{\Gamma(k)} \int_{0}^{\infty} s^{k-1} H(s) d s \\
& \quad+\frac{(-1)^{(k+1) / 2}}{2 \Gamma(k)} \int_{0}^{\infty}\left(\hat{\phi} *\left(|s|^{k-1} \operatorname{sgn} s\right)\right) H(s) d s .
\end{aligned}
$$

Moreover, for the further rewriting of the second term of $(6.95)$, the following formula is useful. 


$$
\begin{aligned}
& \left(\hat{\psi} *\left(|s|^{k-1} \operatorname{sgn} s\right)\right)(s)=\sum_{\substack{p+q=k-1 \\
p \neq \text { odd }}}\left(\begin{array}{c}
k-1 \\
p
\end{array}\right) s^{p}\left\{\int_{s}^{\infty} \widehat{D_{r}^{q} \phi(t)} d t+\int_{-s}^{\infty} \widehat{D_{r}^{q} \psi(t)} d t\right\} \\
& +\sum_{\substack{p+q=k-1 \\
p: \text { even }}}\left(\begin{array}{c}
k-1 \\
p
\end{array}\right) s^{p} \int_{-s}^{s} \widehat{D_{T}^{q} \phi}(t) d t .
\end{aligned}
$$

Now let us set

$$
A_{-j}(s)=\left\{\begin{array}{cc}
a_{-j}(s)=\frac{1}{2}\left(a_{-j}(s)+a_{-j}(-s)\right) & \text { if } j \text { is even, } \\
\frac{1}{i} a_{-j}(s)=\frac{1}{2 i}\left(a_{-j}(s)-a_{-j}(s)\right) & \text { if } j \text { is odd, } \\
\phi_{j}(r)=1-\chi_{j}(r) &
\end{array}\right.
$$

and

$$
n_{j}= \begin{cases}{\left[\frac{j-m+1}{2}\right]} & \text { if } m \text { is odd, } \\ {\left[\frac{j-m+2}{2}\right]} & \text { if } m \text { is even. }\end{cases}
$$

Then, observing Lemmas $6.3,6.4$ and using the above results properly, we get

LEMMA 6.8.

(1) If $m$ is odd, then (6.83) equals to

(6.100) $\frac{\pi(-1)^{n} \jmath}{(j-m) !} \int_{0}^{\infty} e^{-(1-\cos s) / 2 t} s^{j-m} A_{-j}(s) d s$

$$
\begin{aligned}
& -\frac{(-1)^{n_{j}}}{2(j-m) !} \sum_{\substack{p+q=j-m \\
q: \text { even }}}\left(\begin{array}{c}
\jmath-m \\
p
\end{array}\right) \int_{0}^{\infty} e^{-(1-\cos s) / 2 t}\left\{\int_{-s}^{s} \widehat{D_{\tau}^{q} \phi_{j}}(t) d t\right\} s^{p} A_{-j}(s) d s \\
& -\frac{(-1)^{n_{j}}}{2(\jmath-m) !} \sum_{\substack{p+q=j-m \\
q: \text { odd }}}\left(\begin{array}{c}
j-m \\
p
\end{array}\right) \int_{0}^{\infty} e^{-(1-\cos s) / 2 t}\left\{\int_{s}^{\infty} \widehat{D}_{r}^{q} \psi_{j}(t) d t\right. \\
& \left.+\int_{-s}^{\infty} \widehat{D_{r}^{q} \phi_{\jmath}}(t) d t\right\} s^{p} A_{-\jmath}(s) d s .
\end{aligned}
$$

(2) If $m$ is even, then (6.83) equals to

(6.101) $\frac{(-1)^{n},}{(y-m) !} 2 \int_{0}^{\infty} e^{-(1-\cos s) / 2 t} s^{j-m} A_{-j}(s) \log s d s$

$$
-\frac{(-1)^{n}}{\pi(\jmath-m) !} \sum_{p+q=\jmath-m}(-1)^{q}\left(\begin{array}{c}
\jmath-m \\
p
\end{array}\right) \int_{0}^{\infty} e^{-(1-\cos s) / 2 t}\left(\widehat{D_{r}^{q} \psi_{\jmath}} * \log |s|\right) s^{p} A_{-\jmath}(s) d s .
$$

Let us assign the reference numbers $(6.100)_{1},(6.100)_{2}$ and $(6.100)_{3}$ to the first, second and third terms (or sums) of (6.100), respectively. Similarly, $(6.101)_{1}$ and $(6.101)_{2}$ are defined to be the reference numbers of the first and second terms of (6.101), respectively. 
Now, first, let's deal with the case where $m$ is odd.

Since $f_{j}(s)=s^{j-m} A_{-j}(s)$ is an odd function and $f_{j}^{(n)}(0)=0$ for $n \leqq 2 n_{j}$, observing (6.52) and the remark following it, we know that $(6.100)_{1}$ admits the asymptotic expansion

$$
\frac{\pi(-1)^{n} j}{(j-m) !} \sum_{n=n_{j}}^{\infty} F\left(f_{j}\right)_{2 i+1}^{0,0\rangle} n ! t^{n+1}
$$

Next, if we define

$$
\begin{aligned}
& f_{3, q}(s)= \begin{cases}s^{-(q+1)} \int_{-s}^{s} \widehat{D_{r}^{q} \psi_{j}}(t) d t & \text { if } q \text { is even, } \\
s^{-(q+1)}\left\{\int_{s}^{\infty} \widehat{D_{r}^{q} \psi_{s}}(t) d t+\int_{-s}^{\infty} \widehat{D_{r}^{q} \psi_{j}}(t) d t\right. & \end{cases} \\
& \left.-2 \int_{0}^{\infty} \widehat{D_{r}^{q} \psi_{J}}(t) d t\right\} \text { if } q \text { is odd, }
\end{aligned}
$$

then $(6.100)_{2}+(6.100)_{3}$ equals to

$$
\begin{aligned}
& \left.-\frac{(-1)^{n_{j}}}{2(j-m) !} \sum_{p+q=j-m}\left(\begin{array}{c}
j-m \\
p
\end{array}\right) \int_{0}^{\infty} e^{-(1-\cos s) / 2 t} s^{j-m+1} f_{\jmath, q}(s) A_{-j} s\right) d s \\
& -\frac{(-1)^{n_{j}}}{(j-m) !} \sum_{\substack{p+q=j-m \\
q: \text { odd }}}\left(\begin{array}{c}
j-m \\
p
\end{array}\right)\left[\int_{0}^{\infty} \widehat{D_{r}^{q} \phi_{j}(t)} d t\right] \int_{0}^{\infty} e^{-(1-\cos s) / 2 t} s^{p} A_{-j}(s) d s .
\end{aligned}
$$

Moreover, since $g_{\jmath, q}(s)=s^{j-m+1} f_{\jmath, q}(s) A_{-j}(s)$ is an even function and $g_{3, q}^{(n)}(0)=0$ for $n \leqq 2 n,+1$, the first sum of (6.104) admits the asymptotic expansion

$$
-\frac{(-1)^{n_{j}}}{2(j-m) !} \sum_{p+q=j-m} \sum_{n=n_{j+1}}^{\infty}\left(\begin{array}{c}
\jmath-m \\
p
\end{array}\right) F\left(g_{j, q}\right)_{2 n}^{\langle 0,0\rangle} \Gamma(n+1 / 2\rangle t^{n+1} \cdots
$$

Before dealing with the second sum of (6.104), it will be best to summarize the results already obtained. That is, the formal sum

$$
\frac{1}{(2 \pi)^{m+1} 2 t} \sum_{j>m-1} \int_{S_{x}^{*}, v}\left\{(6.100\rangle_{1}+\text { "the first sum of }\{6.104) "\right.
$$

admits an asymptotic expansion of the form

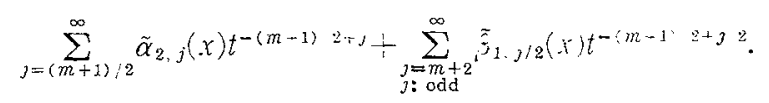

Now, let us deal with the second sum of $(6.104)$. Of course, it admits such an asymptotic expansion as

$$
\sum_{n=1}^{\infty} F_{n, J} t^{n+1 / 2}
$$

However, at this stage, I cannot say which the lowest term in $(6.108)$, is, that is, which the first non-zero term is. Hence, in order to show that the formal sum 


$$
\frac{1}{(2 \pi)^{m-1} 2 t} \sum_{j>m-1} \int_{S_{i}^{*} N} \text { "the second sum of }(6.104) "
$$

admits an asymptotic expansion, we have to carefully investigate whether the formal summation of the (6.108), 's over $j>m-1$ has a sense or not.

The formal sum

$$
\sum_{j, m-1} \text { "the second sum of }(6.104) "
$$

equals to

$$
\int_{11}^{\infty} e^{-\pi-\cos s\rangle / 2 t} f(s) d s
$$

where

$$
\begin{aligned}
& f(s)=\sum_{j=11} \frac{s^{p}}{p^{1}} f_{p}(s)
\end{aligned}
$$

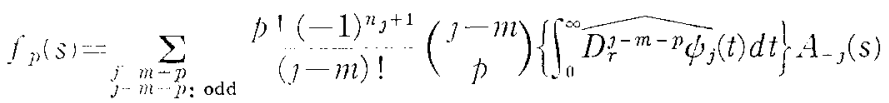

Moreover, if $q$ is odd, then

$$
\begin{aligned}
& \int_{11}^{\infty} \widehat{D_{r}^{q}},(t) d t=-\int_{0}^{\infty} \widehat{D_{r}^{q} \chi_{j}}(t) d t=\int_{-\infty}^{\infty} t_{-}^{q-1} \widehat{D_{r} \chi_{j}}(t) d t \\
& \therefore \int_{-:=}^{\infty} \Phi\left(t^{q-1}\right)(r) D_{r} \chi_{j}(r) d r=(-1)^{(q+1) / 2} \Gamma(q) \int_{-\infty}^{\infty} r^{-q} \chi_{j}^{\prime}(r) d r \\
& =(-1)^{(1,-1) / 2} 2 \Gamma(q) \int_{0}^{\infty} r^{-q \chi_{j}^{\prime}(r) d r} \\
& =(\cdots-1)^{(i,-1) / 2} 2 I(q) M_{j}^{-1} \int_{0}^{\infty} r^{-q} \chi^{\prime}\left(r / M_{j}\right) d r \\
& =(-1)^{(q-1) / 2} 2 I(q) M_{j}^{-q} \int_{0}^{\infty} r^{-q} \chi^{\prime}(r) d r \\
& =I(q) C(q) M_{j}^{-q} \text {, }
\end{aligned}
$$

and

$$
|C(q)| \leqq 2 \int_{0}^{\infty} r^{-\imath \chi^{\prime}}(r) d r \leqq 2 \int_{0}^{\infty} \chi^{\prime}(r) d r=2,
$$

that is, $C(q)$ is bounded independently of $q$; see (6.23). Thus

$$
j_{p}(s)=\sum_{\substack{j>m-p \\ j \cdots m-p ; \text { odd }}} \frac{(-1)^{n_{j+1}} C(j-m-p)}{j-m-p} \cdot \frac{A_{j}(s)}{M_{j}^{j-m-p}} \cdot
$$

Here, it is obrious that, for given $N \in N$, we can find such a sequence $\left\{M_{j}\right\}$ that $\sum_{j=0}^{\infty} M_{j}^{-1} A_{-j}(s)$ and its termuise dervatives of order $\leqq N+1$ converge absolutely near 
$s=0$. Hence, if $N \in N$ is given and such a sequence $\left\{M_{j}\right\}$ is fixed, then the above is true for $f_{p}(s)$ and, moreover, true for $f(s)$. Thus, $f(s)$ which is defined through such a sequence $\left\{M_{j}\right\}$ is of class $C^{\mathrm{v}+1}$ and (6.111) admits an asymptotic expansion of the form

$$
\sum_{1 \leqslant n \leq N / 2} \Gamma(n+1 / 2) F(f)_{2 i}^{\langle 0,(0)} t^{n+1 ; 2} \cdots O\left(t^{(x-2)}{ }^{2}\right)
$$

Hence, given such a sequence $\left\{M_{\}}\right\},(6.109)$ admits an asymptotic expansion of the form

$$
\sum_{\substack{m i 2<j \leqslant m<x \\ j: \text { odd }}} \tilde{3}_{2, j / 2}(x) t^{-(m+1) / 2+j / 2+1}-O\left(t^{-x, 2)}\right.
$$

By the way, $\left\{\varepsilon_{j / 2}(x)\right\},\left\{\tilde{\beta}_{1 j / 2}(x)\right\}$ and this $\left\{\tilde{\tilde{s}}_{2, j / 2}(x)\right\}$ are dependent upon the choice of $\left\{M_{j}\right\}$; see $(6.82),(6.107)$ and $(6.116)$. The summations $\tilde{?}_{j / 2}(x)=\varepsilon_{j / 2}(x)$ $+\tilde{\beta}_{1, j / 2}(x)+\tilde{\beta}_{2, j / 2}(x)$, however, are independent of its choice and produce the second series which appears in the right hand side of (6.55).

Thus, in the case where $m$ is odd, gathering the results (6.i5), (6.77), (6.82), (6.107) and (6.116), we can conclude that $\operatorname{tr} Z(t, x)$ admits the asymptotic expansion (6.55) with (6.56).

Next, we will deal with the case where $m$ is even, that is. (6.101).

Let's decompose $(6.101)_{1}$ into

$$
\begin{aligned}
& \int_{0}^{\infty} e^{-(1-\cos s) / 2 t} s^{\jmath-m} A_{-\jmath}(s) \log \left(\frac{s}{\sin \frac{s}{2}}\right) d s \\
& +\int_{0}^{\infty} e^{-(1-\cos s) / 2 t} s^{\jmath-m} A_{-\jmath}(s) \log \left(\sin \frac{s}{2}\right) d s
\end{aligned}
$$

About the first term, since $\log \left(\frac{s}{\sin \frac{s}{2}}\right)$ is smooth near $s=0$ and $f_{s}(s)=$ $s^{3-m} A_{-},(s) \log \left(\frac{s}{\sin \frac{s}{2}}\right)$ is an even function and $f_{3}^{(n)}(0)=0$ for $n<2 n_{3}$, it admits an asymptotic expansion of the form

$$
\sum_{n=n_{j}}^{\infty} \Gamma(n+1 / 2) F\left(f_{j}\right)_{2 n}^{0,0 ;} t^{n+1 ; 2}
$$

About the second term, setting $g_{j}(s)=s^{j-m} A_{-j}(s)$ and changing the variable $z=\frac{1-\cos s}{2 t}$, it equals to

$$
\begin{aligned}
& t^{1 / 2} \int_{0}^{1 / 2 t} e^{-z} \frac{\log (t z)^{1 / 2}}{z^{1 / 2}(1-t z)^{1 / 2}} g_{J}(\arccos (1-2 t z)) d z \\
= & \left.\frac{1}{2} t^{1 / 2} \int_{0}^{1 / 2 t} e^{-z} z^{1 / 2-1}(1-t z)^{-1 / 2} \log z g_{j}(\arccos 1-2 t z)\right) d z
\end{aligned}
$$




$$
+\frac{1}{2} \log t \int_{0}^{\infty} e^{-(1-\cos s) / 2 t} g_{j}(s) d s .
$$

By the argument similar to Subsection 6.2 (the $\Gamma$-function should be replaced by its derivative), the first term of (6.119) admits an asymptotic expansion of the form $\sum_{n=n_{j}}^{\infty} F_{n, t^{n+1}}$. Moreover the second term admits an asymptotic expansion of the form $\sum_{n=n}^{\infty} G_{n, t^{t}} t^{n+1 / 2} \log t$. Hence, the formal sum

$$
\frac{1}{(2 \pi)^{m+1} 2 t} \sum_{3>m-1} \int_{S_{x}^{*} N}(6.101)_{1}
$$

admits an asymptotic expansion of the form

$$
\sum_{j=2 n / 2+1}^{\infty} \tilde{e} \tilde{\kappa}_{2, j}(x) t^{-(m+1) / 2+\jmath}+\sum_{j=m / 2+1}^{\infty} \tilde{\gamma}_{j}(x) t^{-(m+1) / 2+\jmath} \log t .
$$

Next, let's deal with $(6.101)_{2}$. Owing to the circumstances similar to those of the second term of (6.104), it is no use expanding $(6.101)_{2}$ one by one. Let's decompose $\sum_{j, m-1}(6.101)_{2}$ as follows. For a positive integer $N$,

$$
\begin{aligned}
& \sum_{>m-1}(6.101)_{2}=h_{1, v}(t, x)+h_{2, x}(t, x) \\
& h_{1,1} ! t, x^{n}=\sum_{j=m}^{N+m-1} \frac{(-1)^{n_{j+1}}}{\pi(j-m) !} \sum_{p+q=y-m}(-1)^{q}\left(\begin{array}{c}
j-m \\
p
\end{array}\right)
\end{aligned}
$$

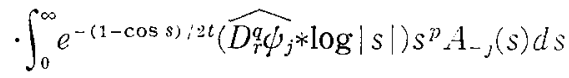

$$
\begin{aligned}
& -\sum_{j=i \neq m}^{\infty} \frac{(-1)^{n j+1}}{\pi(j-m) ! p=j-m-N+1} \sum_{m=-m}^{j-1)^{j-m-p}}\left(\begin{array}{c}
j-m \\
p
\end{array}\right) \\
& \cdot \int_{0}^{\infty} e^{-(1-\cos s) / 2 t}\left(\widehat{D_{r}^{p-m-p} \phi_{j}} * \log |s|\right) s^{p}{ }_{-} L_{-j}(s) d s, \\
& l \times s=\sum_{,=N+m}^{\infty} \frac{(-1)^{n} j+1}{\pi(\jmath-m) !} \sum_{p=0}^{-m-N}(-1)^{\nu-m-p}\left(\begin{array}{c}
\jmath-m \\
p
\end{array}\right) \\
& \cdot\left(\widehat{D_{r}^{p-m-p}} \dot{\psi}_{j} * \log |s|\right) s^{p} A_{-}(s)
\end{aligned}
$$

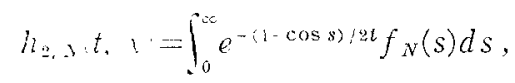

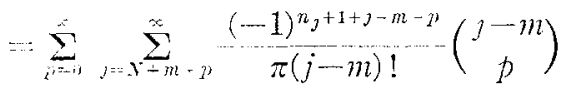

$$
\begin{aligned}
& \left.\widehat{\left(D_{r}^{p-m-p} \phi_{j}\right.} * \log |s|\right) s^{p} 1_{-j}(s) \text {. }
\end{aligned}
$$

Then, it 15 obrious that the first sum of the right hand side of (6.123) 
admits an asymptotic expansion $\sum_{n=1}^{\infty} F_{n} t^{n+1 / 2}$. As for the second sum of the right hand side of (6.123), since $f_{j, p}(s)=\left(\widehat{D_{r}^{p-m-p} \psi_{j}} * \log |s|\right) s^{p} A_{-j}(s)$ is an even function and $f_{j, p}^{(n)}(0)=0$ for $n \leqq j-m-N+1, \int_{0}^{\infty} e^{-(1-\cos s) / 2 t} f_{\jmath, p}(s) d s$ admits an asymptotic expansion of the form

$$
\sum_{n \geq[(\jmath-m-N+2) / 2]} F_{n, j} t^{n+1 / 2}
$$

Hence, the second sum also admits an asymptotic expansion. Thus, we get

$$
\frac{1}{(2 \pi)^{m+1}} 2 t \int_{S_{x}^{*} N} h_{1, N}(t, x) \sim \sum_{j=m / 2+1} \tilde{\alpha}_{s, j}(x) t^{-(m+1) / 2+\jmath} .
$$

In order to show that

$$
\frac{1}{(2 \pi)^{m+1} 2 t} \int_{S_{x}^{*} N} h_{2, N}(t, x)
$$

admits an asymptotic expansion, some preparations are needed. If $q \geq N$, then

$$
\begin{aligned}
& \widehat{D_{r}^{q} \phi_{j}} * \log |s|=\widehat{D_{r}^{q} \psi_{j}} *\left(-\gamma-\frac{1}{2} \mathscr{I}\left(|r|^{-1}\right)\right) \\
= & -2 \pi \gamma D_{r}^{q} \psi_{j}(0)-\pi \mathscr{I}\left(|r|^{-1} D_{r}^{q} \psi_{j}(r)\right) \\
= & -\pi i^{-q} \int_{-\infty}^{\infty} e^{-\imath s r}|r|^{-1} \phi_{j}^{(q)}(r) d r \\
= & \pi i^{-q} \int_{-\infty}^{\infty} e^{-\imath s r}|r|^{-1} \chi_{j}^{(q)}(r) d r \\
= & \pi \imath^{-q}(-1)^{q-N} \int_{-\infty}^{\infty}\left(\frac{\partial}{\partial r}\right)^{q-N}\left(e^{-\imath s r}|r|^{-1}\right) \chi_{j}^{(N)}(r) d r \\
= & \pi \sum_{k=0}^{q-N} s^{k} \frac{(q-N) !}{k !} i^{k-q} \int_{-\infty}^{\infty} e^{-\imath s r} r^{-(q-k+N+1)} \operatorname{sgn} r \chi_{j}^{(N)}(r) d r \\
= & \pi \sum_{k=0}^{q-N} s^{k} \frac{(q-N) !}{k !} M_{j}^{-(q-k)} i^{k-q} \int_{-\infty}^{\infty} e^{-\imath s r M_{j}} r^{-(q-k+N+1)} \operatorname{sgn} r \chi^{(N)}(r) d r \\
= & \pi \sum_{k=0}^{q-N} s^{k} \frac{q !}{k !}(-1)^{n_{j}+1+q} M_{j}^{-(q-k)} h(s: N, \jmath, q, k),
\end{aligned}
$$

where

$$
h(s: N, j, q, k)=(-1)^{n_{\jmath}+1+q} \frac{(q-N) !}{q !} i^{k-q} \int_{-\infty}^{\infty} e^{-\imath s r M_{\jmath}} r^{-(q-k+N+1)} \operatorname{sgn} r \chi^{(N)}(r) d r .
$$

Hence, $f_{N}(s)$, that is, (6.125), equals to

(6.129) $\sum_{p=0}^{\infty} \sum_{\jmath=N+m+p}^{\infty} \sum_{p=0}^{\jmath-N-p} s^{p+k} M_{\jmath}^{-(\jmath-m-p-k)} h(s: N, \jmath, \jmath-m-p, k) A_{-j}(s)$ 


$$
\begin{aligned}
& =\sum_{l=0}^{\infty} \sum_{\jmath=\Lambda^{+}+m+p}^{\infty} \sum_{l=p}^{v-m} \frac{1}{p !(l-p) !} s^{l} M_{\jmath}^{-(\jmath-m-l)} h(s: N, j, \jmath-m-p, l-p) A_{-\jmath}(s) \\
& =\sum_{l=0}^{\infty} \frac{(2 s)^{l}}{l !} f_{N, l}(s)
\end{aligned}
$$

where

$$
f_{x, l}(s)=\sum_{\jmath=N+m+l}^{\infty} \sum_{p=0}^{l} 2^{-l}\left(\begin{array}{l}
l \\
p
\end{array}\right) \frac{h(s: N, j, j-m-p, l-p) A_{-\jmath}(s)}{M_{j}^{p-m-l}} .
$$

Given $n<N$, we have

$$
\begin{aligned}
& \left|f_{i}^{(n)}, l(s)\right| \leqq \sum_{k=0}^{n}\left(\begin{array}{l}
n \\
k
\end{array}\right) \sum_{j=.+\cdots+m+l}^{\infty} \sum_{p=0}^{l} 2^{-l}\left(\begin{array}{l}
l \\
p
\end{array}\right) \frac{\int_{-\infty}^{\infty}\left|r^{k+\jmath+N-m-l-1} \chi^{(N)}(r)\right| d r A_{-j}^{(n-k)}(s)}{M_{j}^{j-m-l}} \\
& \leqq \sum_{k=0}^{n} 2^{k+x} C_{N}\left(\begin{array}{l}
n \\
k
\end{array}\right) \sum_{j=N+m+l}^{\infty} \frac{\left|A_{-j}^{(n-k)}(s)\right|}{M_{j}^{j-m-l}-k} \\
& \leqq \sum_{k=0}^{n} 2^{k+v} C_{N}\left(\begin{array}{l}
n \\
k
\end{array}\right)_{j=N+m+l} \sum_{j=1}^{\infty} \frac{\left|A_{-j}^{(n-k)}(s)\right|}{M_{\jmath}} \\
& \leqq 2^{3 . Y} C_{Y} \sum_{j=Y+m}^{\infty} M_{j}^{-1} \max _{0 \leqq n \leqq N}\left|A_{-j}^{(n)}(s)\right|,
\end{aligned}
$$

where $C_{N}=\int_{-\infty}^{\infty}\left|\chi^{(N)}(r)\right| d r$. Hence, we can choose such a sequence $\left\{M_{j}\right\}$ that $f_{N, l}(s)$ is of class $C^{X-1}$. Moreover, $f_{N}(s)$ which is defined through such $\left\{M_{j}\right\}$ is also of class $C^{N-1}$. Observing that $f_{N}(s)$ is an even function and $f_{N}(0)=0$, we know that (6.127) admits an asymptotic expansion

$$
\sum_{m / 2+1 \leq j \leq\{N-3) / 2} \tilde{a}_{4, j}(x) t^{-(m+1) / 2+j}+O\left(t^{N / 2-1}\right) .
$$

By the way, $\left\{\varepsilon_{j}(x)\right\},\left\{\tilde{\alpha}_{2, j}(x)\right\},\left\{\tilde{\alpha}_{s, j}(x)\right\}$ and $\left\{\tilde{\alpha}_{4,3}(x)\right\}$ are dependent upon the choice of $\left\{M_{j}\right\}$; see $(6.82),(6.121),(6.126)$ and $(6.131)$. The summations $\tilde{\alpha}_{j}(x)=$ $\tilde{\alpha}_{1, j}(x)+\varepsilon_{j}(x)+\tilde{\alpha}_{2, j}(x)+\tilde{\alpha}_{3, j}(x)+\tilde{\alpha}_{1, j}(x)$, however, are independent of its choice and produce the first series which appears in the right hand side of (6.55).

Thus, in the case where $m$ is even, gathering the results (6.76), (6.77), (6.82), (6.121), (6.126) and $(6.131)$, we can conclude that $\operatorname{tr} Z(t, x)$ admits the asymptotic expansion (6.55) with (6.56).

\section{REFERENCES}

1. J. Chemger, Analytic torsion and the heat equation, Ann. Math., 109 (1979), 259. 322.

2] J. CheEger, On the spectral geometry of spaces with cone-like singularities, P. N. A.S., V. 76, No. 5, (1979), 2103-2106.

3 J. J. Cheeger, Spectral geometry of spaces with cone-like singularities, preprint, (1978). 
4. J. Cinbscer, On the Hodge theory of Riemannan pseudomanifolds, Proc. Sym. Pure Math., 36 (1980), 91-146.

5 .. J. Cineeger, M. Goresky axd R. MacPiersox, $L^{2}$-cohomology and intersection homology of singular algebraic varieties, in Proceeding of year in differential geometry, I. A.S., S. Yau ed. (1981), Ann. Math. Studies, Princeton.

-6! J. CheEger AND M. TAlLor, On the diffraction of waves by conical singularities I, If, Comm. Pure Appl. Math., XXXV (1982), 275-331: XXXV (1982), 487-529.

7- P.E. CoNier, The Neumann's problem for differential forms on Riemannian manifolds, Mem. Amer. Math. Soc., 20 (1956).

8 J. Dutstermant AND V. Gulliemix, The spectrum of positive elliptic operators and periodic bicharacteristics, Invent. Math., 20 (1975), 39-79.

9- M.P. GAFFXEY, A special Stokes theorem for Riemannian manifolds, Ann. Math., V. 60, No. 1, (1954), 140-145.

10] M.P. GAFFNEY, The heat equation method of Milgram and Rosenbloom for open Riemannian manifolds, Ann. Math., V. 60, No. 3, (1954), 458-466.

11] P. GRELNER, An asymptotic expansion for the heat equation, Arch. Rat. Mech. Anal., 41 (1971), 163-218.

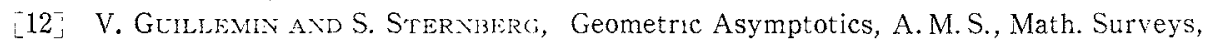
No. 14 (1977).

i13] L. Hörmaxder, The spectral function of an elliptic operator, Acta. Math., 121 (1968), 193-218.

[14] S. Moriglte, K. Udagaira axd S. Hrtotcmate, Sūgaku Kōshikı I, II, III, Iwanami Shoten (in Japanese).

[15] M. NAGASE, De Rham-Hodge theory on a manifold with cone-like singularities, Kodai Math. J., V. 5, No. 1, (1982), 38-64.

[16] M. NAGASE, $L^{2}$-cohomology and intersection homology of stratified spaces, Duke Math. J., V. 50, No. 1, (1983), 329-368.

-17] D. B. RAY AND I. M. Sixger, R-torsion and the Laplacian on Riemannian maifolds, Adv. in Math., 7 (1971), 145-210.

18? M. TAYroR, Pseudodifferential Operators, Princeton Univ. Press, (1981).

Departaneat of Mathematics

Tokyo INstitute of Teciñology

Oh-okayama, Meglro-ke, Tokyo

JAPAXX 
